\title{
COMPARATIVE STUDY OF SEMISYNTHETIC DERIVATIVE OF NATAMYCIN AND THE PARENT ANTIBIOTIC ON THE SPOILAGE OF SHREDDED CHEDDAR CHEESE
}

\author{
By
}

Eric C. Suloff

Thesis submitted to the faculty of the Virginia Polytechnic Institute and State University in partial fulfillment of the requirements for the degree of

Master of Science

In

Food Science and Technology

Approved:

Dr. Joseph E. Marcy, Chairman

Dr. Cameron R. Hackney

Dr. Susan S. Sumner

2 December 1999

Blacksburg, Virginia

Keywords: Pimaricin, Antibiotic, Antimycotic, Antifungal, Fungicide, Preservative, Fungi

Copyright 1999, Eric C. Suloff 


\title{
COMPARATIVE STUDY OF SEMISYNTHETIC DERIVATIVE OF NATAMYCIN AND THE PARENT ANTIBIOTIC ON THE SPOILAGE OF SHREDDED CHEDDAR CHEESE
}

\section{By}

\author{
Eric C. Suloff
}

\begin{abstract}
The polyene macrolide antibiotic natamycin (Antibiotic A-5283) is commonly used to retard the growth of surface molds on various cheese varieties. Natamycin is commonly applied to the surface of cheese by dipping or spraying, using an aqueous dispersion containing 200 to $300 \mathrm{ppm}$ of the additive. The large molecular weight of natamycin, $666 \mathrm{~g} / \mathrm{mol}$, and conjugated double bond structure causes it to be extremely insoluble in water and most food grade solvents. The inability to apply natamycin in true solution creates void non-treated areas on the food surface. These non-treated areas promote the growth of fungal organisms.

A water soluble $N$-alkyl semisynthetic derivative of natamycin was synthesized by the Michael addition reaction of the parent with a $N$-substituted malemide. A comparative study investigating the effectiveness of the semisynthetic derivative of natamycin and the parent antibiotic in suppressing mold growth on one month aged shredded Cheddar cheese modified atmosphere packaged (MAP) was performed. A $20 \mathrm{ppm}$ natamycin treatment effectively suppressed visible mold growth $\left(<10^{4} \mathrm{CFU} / \mathrm{g}\right)$ in MAP samples for up to 30 days after opening. The 20 ppm semisynthetic derivative performed similarly to the $10 \mathrm{ppm}$ natamycin treatment in retarding mold growth. Visible mold growth did not occur for these treatments in MAP samples until 20 days after opening. Analysis of storage conditions revealed that an outgrowth of mold in shredded cheese occurred in MAP packages stored longer than 15 days. This bloom in mold growth was attributed to the degradation of natamycin and the semisynthetic derivative throughout storage.

The stability and degradation of natamycin and the derivative were monitored throughout the study. Antibiotic concentration on the cheese surface was quantified by molecular absorption spectrometry. Results from this study showed, heavily contaminated samples caused the rate and loss of natamycin and the derivative to increase. Antibiotic concentration decreased at a similar rate in MAP and open package conditions. Natamycin and derivative were found to have similar degradation properties.
\end{abstract}




\section{DEDICATION}

This thesis is dedicated to my wife, Amy Lynn Suloff. I cannot express in words the love and support Amy provided during this project. For these endearing qualities, and so many more I want to thank her. 


\section{ACKNOWLEDGMENTS}

I wish to express my sincere gratitude to my major advisor, Dr. Joseph E. Marcy, for his suggestions, guidance, patience, understanding, and assistance which, without, my research work would not have been possible.

I would like to extend further appreciation to my committee members, Dr. Cameron R. Hackney and Dr. Susan Sumner, for their interest, advisement and input throughout my research work.

Very special thanks to Dr. David G. Kingston and Dr. Prakash G. Jagtap for their assistance with the synthesis of the natamycin derivative, to Tom Glass and Ann Campbell for their help in mass spectroscopy and NMR analysis, to Laura Sammons for her advice and guidance of fungal spoilage organisms, to Walter Hartman and John Chandler for their help during the processing of cheese, to Brian Yaun, Lauren Knezovich and Wes Smittle for their endless hours of support in the lab, and Harriet Williams for her constant support.

Additionally, I want to recognize the Nebraska Center for Mass Spectrometry for their analysis of the natamycin derivative and the Wisconsin Milk Marketing Board for financially supporting this research. 


\section{CONTENTS}

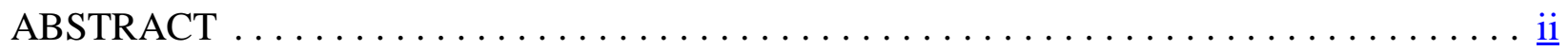

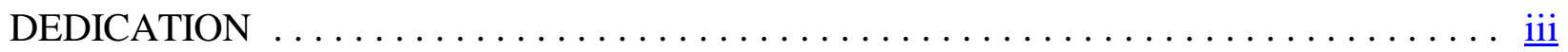

ACKNOWLEDGMENTS $\ldots \ldots \ldots \ldots \ldots \ldots \ldots \ldots \ldots \ldots \ldots \ldots \ldots \ldots$

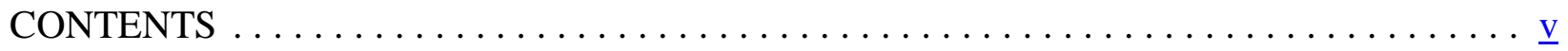

CHAPTER 1: REVIEW OF LITERATURE $\ldots \ldots \ldots \ldots \ldots \ldots \ldots \ldots \ldots \ldots \ldots \ldots \ldots$

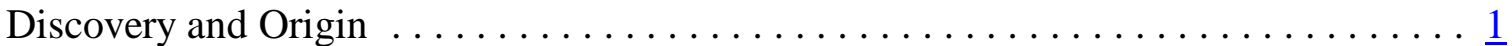

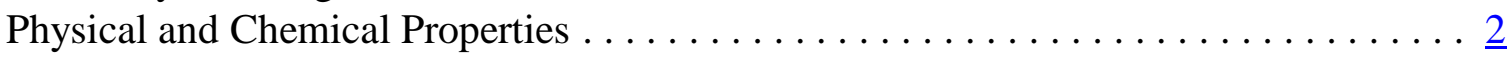

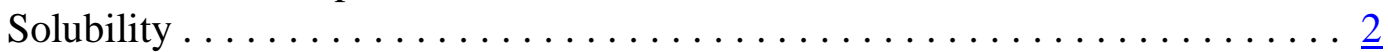

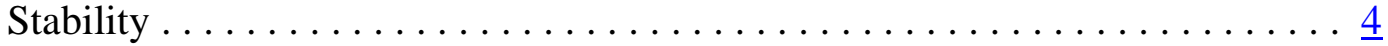

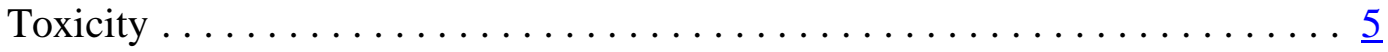

Derivatives of Polyene Macrolide Antibiotics $\ldots \ldots \ldots \ldots \ldots \ldots \ldots \ldots \ldots \ldots \ldots \ldots \ldots$

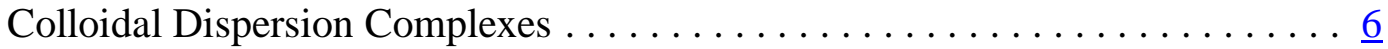

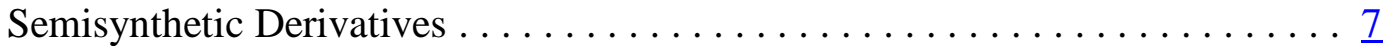

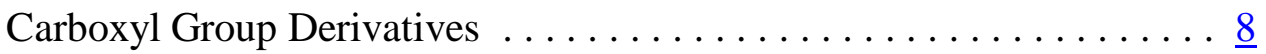

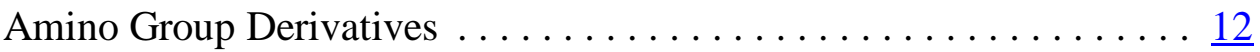

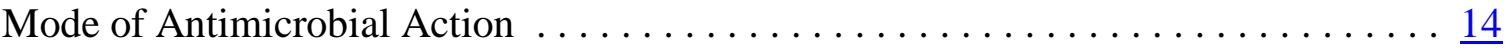

Resistance and Tolerance ........................... 17

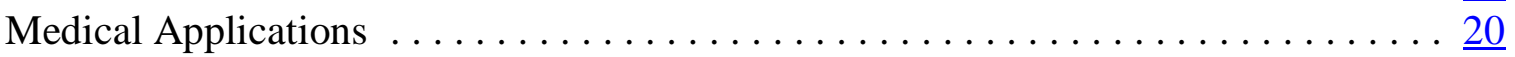

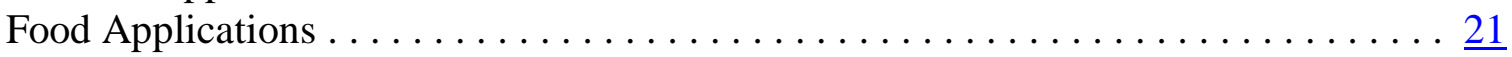

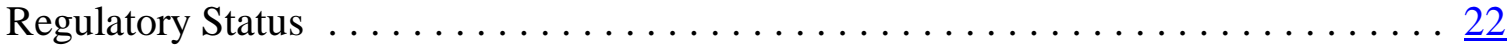

Cheese Spoilage Organisms . . . . . . . . . . . . . . . . . . 24

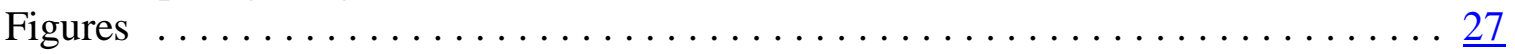

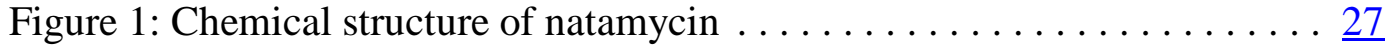

Figure 2: Natamycin crystals (dissolved in vegetable oil at 500X magnification)

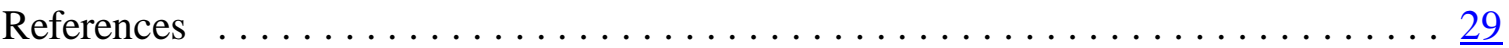

CHAPTER 2: SYNTHESIS OF $N$-AMINOACYL DERIVATIVE OF NATAMYCIN $\ldots \ldots \underline{42}$

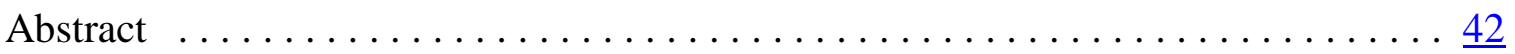

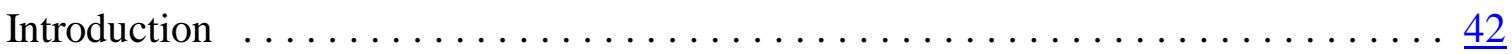

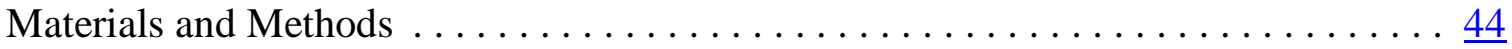

Synthesis of Propylaminomalemide (PAM) Hydrochloride . . . . . . . . 44

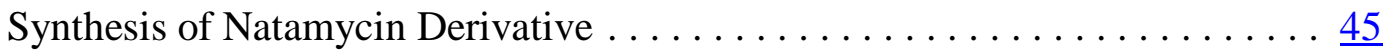

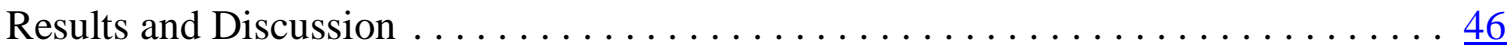

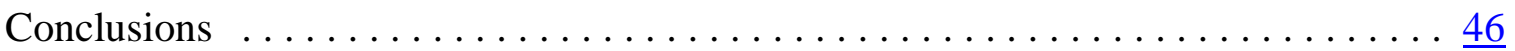

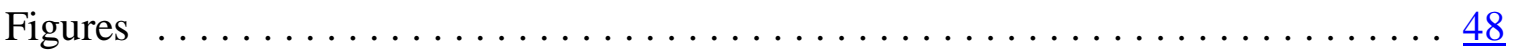


Figure 1: Major ions present in the positive ion FAB mass spectras for natamycin, PAM hydrochloride, and natamycin derivative $\ldots \ldots \ldots \ldots \ldots$

Figure 2: ${ }^{1} \mathrm{H}$ NMR spectral data for PAM hydrochloride and natamycin derivati $\underline{8}$ References $\underline{49}$

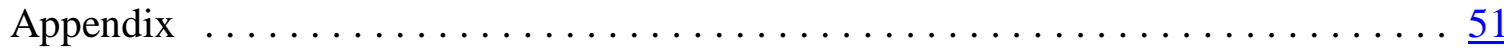

Figure 1: Reaction scheme for the preparation of propylaminomalemide (PAM)

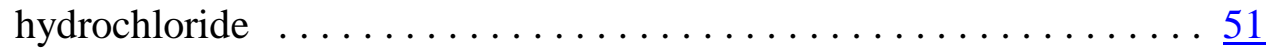

Figure 2: Reaction scheme for the preparation of N-(3'-Ndimethylaminopropylsuccimido) natamycin (natamycin derivative) $\ldots \underline{52}$

Figure 3: Low resolution FAB mass spectra of natamycin . . . . . . . . $\underline{53}$ Figure 4: Low resolution FAB mass spectra of PAM hydrochloride $\ldots \ldots \underline{54}$ Figure 5: ${ }^{1} \mathrm{H}-\mathrm{NMR}$ spectra of PAM hydrochloride in $\mathrm{D}_{2} \mathrm{O} \ldots \ldots \ldots \ldots \ldots$ Figure 6: Low resolution FAB mass spectra of N-(3'-Ndimethylaminopropylsuccimido) natamycin ............ $\underline{56}$

Figure 7: High resolution FAB mass spectra of N-(3'-Ndimethylaminopropylsuccimido) natamycin (natamycin derivative) . $\underline{57}$

Figure 8: ${ }^{1} \mathrm{H}-\mathrm{NMR}$ spectra of $\mathrm{N}$-(3'-N-dimethylaminopropylsuccimido) natamycin (natamycin derivative) in pyridine- $\mathrm{d}_{5} \ldots \ldots \ldots \underline{58}$

\section{CHAPTER 3: MICROBIOLOGICAL CHALLENGE STUDY FOR SEMISYNTHETIC DERIVATIVE OF NATAMYCIN AND PARENT ANTIBIOTIC . . . . . . . . $\underline{59}$}

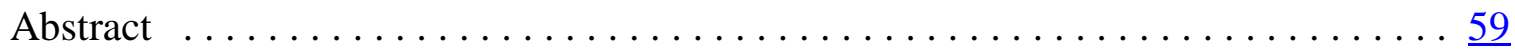

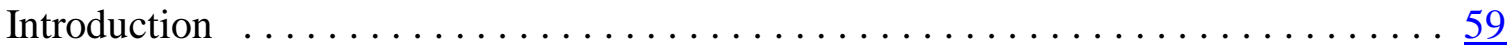

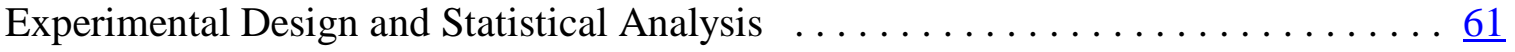

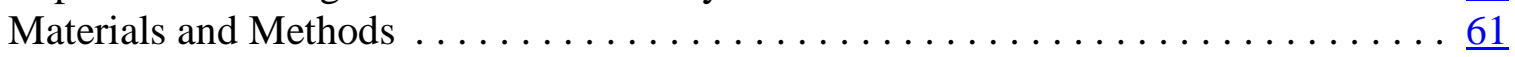

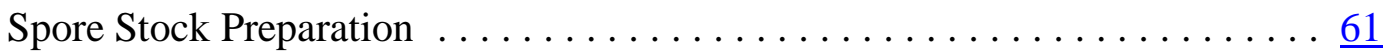

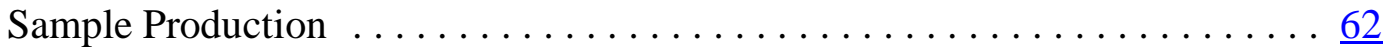

Microbiological Sampling . . . . . . . . . . . . . . . . . . 63

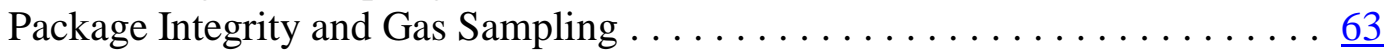

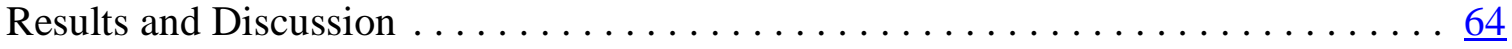

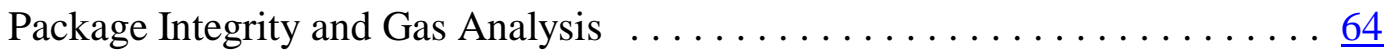

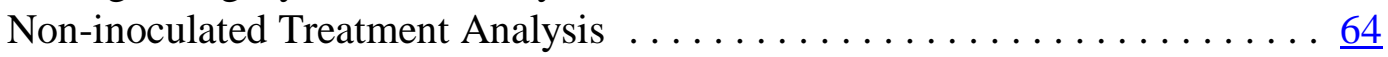

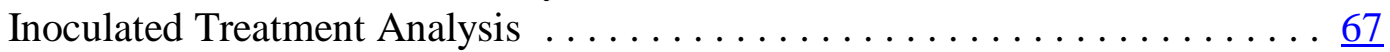

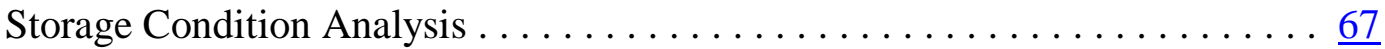

Conclusions ......................

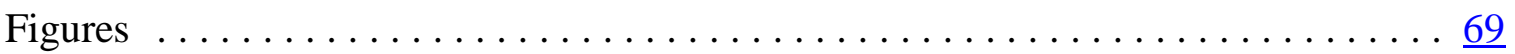

Figure 1: Mold growth on 1 month aged shredded Cheddar cheese at various storage conditions and antibiotic treatments .......... $\underline{69}$

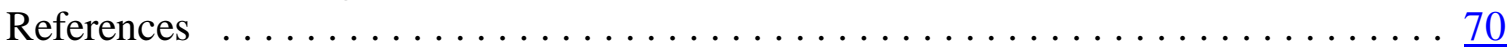

Appendix . . . . . . . . . . . . . . .

Figure 1: Application treatments for microbiological challenge study of semisynthetic derivative of natamycin and parent antibiotic $\ldots \ldots \underline{72}$ 
Figure 2: Storage treatments for microbiological challenge study of semisynthetic derivative of natamycin and parent antibiotic . . . . . . .

Figure 3: Statistical model for microbiological challenge study of semisynthetic derivative of natamycin and parent antibiotic .........

Figure 4: Gas analysis of MAP sample bags for replication $1 \ldots \ldots \ldots \ldots \underline{74}$

Figure 5: Gas analysis of MAP sample bags for replication $2 \ldots \ldots \ldots \ldots \underline{75}$

Figure 6: Gas analysis of MAP sample bags for replication $3 \ldots \ldots \ldots \ldots \underline{76}$

Figure 7: Mold growth on 1 month aged shredded Cheddar cheese for antibiotic treatments vs. storage conditions: 0 days MAP : 0, 10, 20, 30, 40 days open .................... 77

Figure 8: Mold growth on 1 month aged shredded Cheddar cheese for antibiotic treatments vs. storage conditions: 15 days MAP : 0, 10, 20, 30, 40 days

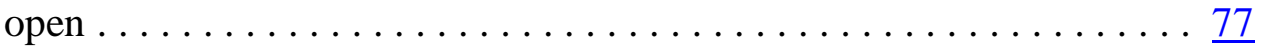

Figure 9: Mold growth on 1 month aged shredded Cheddar cheese for antibiotic treatments vs. storage conditions: 30 days MAP : 0, 10, 20, 30, 40 days open ...................

Figure 10: Mold growth on 1 month ripened shredded Cheddar cheese inoculated with Penicillium roqueforti spores at various storage conditions and

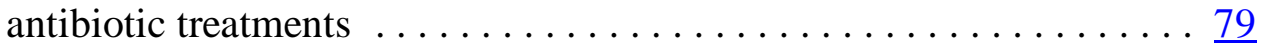

\section{CHAPTER 4: STABILITY CHALLENGE STUDY FOR SEMISYNTHETIC DERIVATIVE OF} NATAMYCIN AND PARENT ANTIBIOTIC $\ldots \ldots \ldots \ldots \ldots \ldots \ldots \ldots \ldots \ldots \ldots \ldots \ldots$

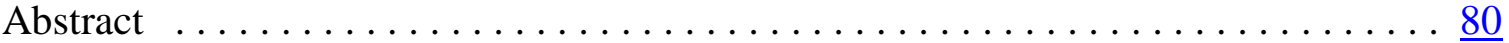

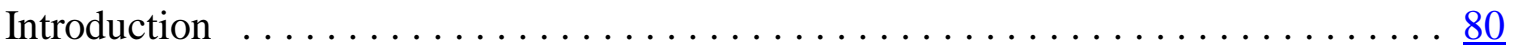

Materials and Methods .......................

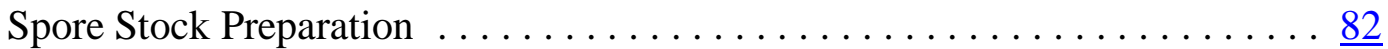

Sample Production ........................ $\underline{83}$

Procedure for Determining Natamycin and Derivative Concentration on Cheese

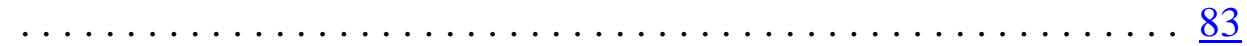

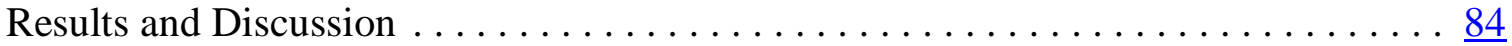

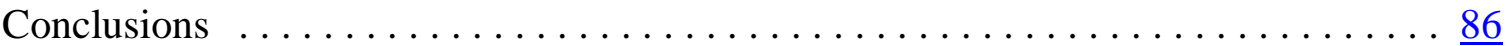

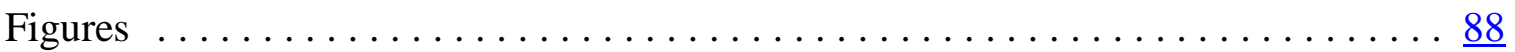

Figure 1: Antibiotic concentration of 1 month aged shredded Cheddar cheese for non-inoculated treatments vs. total days of storage . . . . . . . $\underline{88}$

Figure 2: Antibiotic concentration of 1 month aged shredded Cheddar cheese for inoculated treatments vs. total days of storage $\ldots \ldots \ldots \ldots \ldots \underline{8}$

Figure 5: Antibiotic concentration of 1 month aged shredded Cheddar cheese for non-inoculated treatments at storage condition: 0 Days MAP : 0, 20 Days

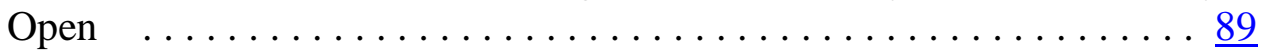

Figure 6: Antibiotic concentration of 1 month aged shredded Cheddar cheese for inoculated treatments at storage conditions: 0 Days MAP : 0, 20 Days

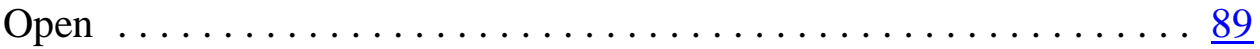


Figure 7: Antibiotic concentration of 1 month aged shredded Cheddar cheese for non-inoculated treatments at storage condition: 15 Days MAP : 0, 20 Days Open . . . . . . . . . . . . . . . . . . . 90

Figure 8: Antibiotic concentration of 1 month aged shredded Cheddar cheese for inoculated treatments at storage condition: 15 Days MAP : 0, 20 Days Open .....................

Figure 9: Antibiotic concentration of 1 month aged shredded Cheddar cheese for non-inoculated treatments at storage condition: 30 Days MAP : 0, 20 Days Open . . . . . . . . . . . . . . . . . . 91

Figure 10: Antibiotic concentration of 1 month aged shredded Cheddar cheese for inoculated treatments at storage condition: 30 Days MAP : 0, 20 Days

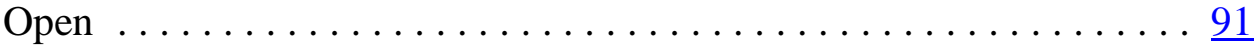

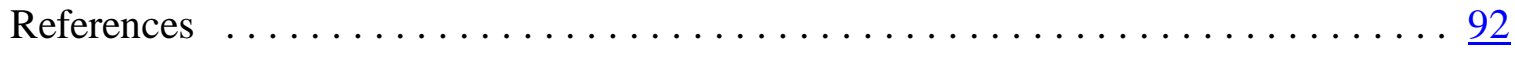




\section{CHAPTER 1: REVIEW OF LITERATURE}

\section{Discovery and Origin}

In 1955, a Streptomyces strain was taken from a soil sample near the town of Pietermaritzburg in Natal, a province of South Africa by Dutch scientists. The Streptomyces strain was named Streptomyces natalensis (Raab, 1972; Thomas, 1976). A new, highly active antibiotic was isolated from culture filtrates of Streptomyces natalensis and was named pimaricin. Gist-brocades B.V. (Delft, The Netherlands) filed the patent application, "Pimaricin and Process of Producing the Same," on March 13, 1956 in the Netherlands (Struyk and Waisvisz, 1960). Gist-brocades B.V. filed the U.S. patent application for pimaricin on March 7, 1957 (Struyk and Waisvisz, 1975).

The antibiotic tennecetin was isolated from a soil sample in Chattanooga, TN from a Streptomyces strain in 1959. The strain was named Streptomyces chattanoogensis. Around the same time, another antibiotic, A 5283, was isolated from Streptomyces gilvosporus. Tennecetin and A 5283 were proved to have the identical chemical structure of pimaricin (Clark et al., 1964). The production of pimaricin from Streptomyces gilvosporus was applied for patent in the U.S. by the American Cyanamid Company on July 23, 1956 (American Cyanamid Company, 1960).

In the late 1960's the World Health Organization (WHO) established a regulation specifying that antibiotics produced from Streptomyces must carry names ending in "...mycin." Therefore, the antibiotic pimaricin changed its name to natamycin (Raab, 1972).

A review of literature shows that natamycin and pimaricin are interchangeably used to describe this antibiotic. The food industry and most of the British Commonwealth use the name natamycin to describe this substance, while most of the rest of the world and medical community use the old name, pimaricin, more prevalently. 


\section{Physical and Chemical Properties}

Natamycin has an empirical formula of $\mathrm{C}_{33} \mathrm{H}_{47} \mathrm{NO}_{13}$ and a molar mass of 665.73 (Lück and Jager, 1997). The International Union of Pure and Applied Chemistry (IUPAC) chemical name for natamycin is 22-[(3-amino-3,6-dideoxy- $\beta$-D-mannopyranosyl)-oxy]-1,3,26-trihydroxy12-methyl-10-oxo-6,11,28-trioxatricyclo[22.3.1.0 $\left.0^{5,7}\right]$ octacosa-8,14,16,18,20-pentaene-25carboxylic acid. Chemical Abstract Service (CAS) registry number is 7681-93-8 (Brik, 1981). The structural formula of natamycin is depicted in Figure 1.

The primary structure of natamycin is its large lactone ring of $25 \mathrm{C}$-atoms. The lactone ring is linked to a mycosamine moiety, amino-sugar, by a glycosidic linkage, classifying natamycin as a polyene macrolide antibiotic. In particular, natamycin is a tetraene antibiotic because of its four conjugated double bonds. The mycosamine (3-amino-3,6-dideoxy-Dmannose) of natamycin at the $\mathrm{C} 15$ position is a six-membered pyranose ring (Thomas, 1976).

Natamycin is a white, tasteless, and odorless powder. The antibiotic is crystalline in structure and melts at a temperature of $180^{\circ} \mathrm{C}$ upon decomposition (Figure 2) (Oostendorp, 1981). Exposures in excess of one hour at temperatures above $120^{\circ} \mathrm{C}$ impair the antibiotic activity of natamycin (Lück and Jager, 1997; Raab, 1972).

\section{Solubility}

Natamycin is extremely insoluble in water. Schaffner and Mechlinski (1972) report the solubility of natamycin as $0.0520 \mathrm{mg} / \mathrm{ml}$. In addition, natamycin is insoluble in higher alcohols, ethers, esters, aromatic or aliphatic hydrocarbons, chlorinated hydrocarbons, ketones, dioxane, cyclohexanol and various oils. Glacial acetic acid, methylpyrrolidone, dimethylformamide, dimethylsulfoxide, glycerol, and propylene glycol are good solvents for natamycin (Raab, 1972).

The large molecular weight, $665.73 \mathrm{~g} / \mathrm{mol}$, and conjugated double bond structure explains the poor solubility of natamycin in water and most alcohol solutions (Thomas, 1976). Natamycin's insolubility in most hydrocarbons is attributed to the three hydroxyl groups in the lactone ring and the formation of a dipolar ion. Natamycin is an amino acid that contains both 
acidic and basic groups that undergo an intermolecular acid-base reaction. This reaction causes natamycin to exist in the form of a dipolar ion, or zwitterion (McMurry, 1992).

The zwitterionic nature of natamycin allows it to react with acids or bases depending upon the circumstance. In an aqueous acid solution, natamycin can accept a proton at the C12 carboxyl group and form a cation. Conversely, in an aqueous basic solution, natamycin loses a proton from its amino group of the mycosamine moiety forming an anion. Amino acids that possess this dual relationship with acids and bases are called amphoteric (McMurry, 1992).

The solubility and amphoteric nature of natamycin can be better understood by viewing this antibiotic in two chains. The chain that contains the four double bonds is completely hydrophobic, whereas the chain with the two hydroxyl groups has a hydrophillic and hydrophobic face, rendering the chain amphipathic. The polar end of the amphipathic chain contains the mycosamine and a carboxyl group. The other end is marginally non-polar with only one hydroxyl group. Natamycin forms a cylindrical structure by aligning hydroxyl groups of its amphipathic chain toward each other. The exterior of the cylinder is completely non-polar (Thomas, 1976).

Thomas (1976) indicates another reason why many polyene antibiotics are extremely insoluble and experience relatively high stability is the existence of a six-membered ketal ring structure when the antibiotic is in a crystalline form. The ketal ring in natamycin is achieved by joining carbon atoms 9 and 13 .

Strong solvent-solute interactions explain the exceptional solubility of natamycin in dimethylformamide and dimethylsulfoxide. Infrared studies by Mechlinski and Schaffner (1972) indicate strong intermolecular hydrogen bonds involving hydroxyl, carboxyl, and amino groups. These studies also suggest disruption of the zwitterion by solvent molecules. This subjects the carboxyl and amino group to chemical reactions.

Natamycin's solubility in methylpyrrolidone and other strong polar organic solvents is not fully understood. It is believed that polyene antibiotic molecules form suspensions of micelles rather then dissolving. Micellular suspensions may also cause polyene antibiotics to exist in pure aqueous solutions without visible precipitation at concentrations of $50 \mu \mathrm{g} / \mathrm{ml}$ (Oostendorp, 1981; Thomas, 1976). 


\section{Stability}

Natamycin is most stable in a crystalline powder form in the absence of heat and light. No loss in biological activity occurs when stored at room temperature and protected from ultraviolet light at 30 months. The stability of natamycin is lower in dry formulations of sodium chloride, citric acid, and sucrose (Hoogerheide, 1961 and 1964, unpublished data).

Micellular suspensions of natamycin offer protection by shielding the labile sites of the molecule (Thomas, 1976). Studies show no loss in biological activity for a $0.5 \%$ aqueous suspension of natamycin with $0.1 \%$ polyoxyethylene stored in the dark at $\mathrm{pH} 6.5$ for 24 months (Hoogerheide, 1961 and 1964, unpublished data).

Ultraviolet light with wavelengths of 300 to $350 \mathrm{~nm}$ quickly inactivates natamycin (Dekker and Ark, 1959). The tetraene structure of natamycin causes its instability in the presence ultraviolet light. The photodynamic destruction of natamycin occurs by a unique triplet-triplet transfer mechanism. Most light induced destructions occur as a result of oxidation or by a free-radical mechanism. A triplet-triplet transfer occurs when a compound in an excited triplet state collides with a compound in the singlet state at a lower energy state. The result is a conversion of spin movement for the singlet state compound causing it to excite the molecule. An experimental demonstration of this phenomenon was conducted for natamycin. Excited riboflavin molecules in the triplet state bombarded natamycin in the 300 to $350 \mathrm{~nm}$ range causing a triplet-triplet transfer. The triplet-triplet transfer caused the natamycin molecule to quickly degrade the reactive tetraene structure (Posthuma and Berends, 1960; 1961). Photodynamic destruction of natamycin caused by triplet-triplet transfer does not show decreases in maxima peaks 279, 290,304, and $318 \mathrm{~nm}$ in an ultraviolet absorption spectra (Thomas, 1976).

Natamycin suspensions and solutions lose their biological activity by oxidation. Oxidation is promoted by elevated temperatures, greater than $37^{\circ} \mathrm{C}$, and in the presence of chemical oxidants (Dekker and Ark, 1959; Barr, 1959). Chemical oxidants such as peroxides, perchlorates, persulfates, permanganates, iodates, bromates, hypochlorites, sulfites, and 
anhydrides of organic acids cause the oxidative inactivation of natamycin. The ultraviolet absorption spectra of natamycin following oxidative inactivation show marked decreases in maxima peaks, 279, 290, 304, and $318 \mathrm{~nm}$ (Thomas, 1976).

Oxidation is amplified at $\mathrm{pH}$ levels below 5.0 and above 9.0 (Raab, 1972). In organic acids, acid hydrolysis occurs resulting in the elimination of the mycosamine. Natamycin changes its structure from a tetraene to a pentaene as a result (Thomas, 1976). Oxidative inactivation may be prevented by the addition of chlorophyll, ascorbic acid, or a number of other antioxidants (Dekker and Ark, 1959; Barr, 1959; Struyk et al., 1958).

The isoelectric point of natamycin is 6.5. Solutions of natamycin with $\mathrm{pH}$ values between 5.0 and 9.0 are stable when stored in the dark (Raab, 1972). Natamycin undergoes hydrogenation in the presence of a palladium catalyst. Single-stage hydrogenation occurs in an alkaline medium, whereas a more complete hydrogenation is demonstrated in neutral or acidic solutions (Raab, 1972).

"Physiochemically, the stability of natamycin depends greatly on the stability of the tetraene moiety of the molecule. Readily occurring inactivation of natamycin (in solution) by heat, by light, (ultraviolet light or in the presence of substances capable of transferring photoenergy) or by oxidation always begins with the severing of these interconnected, unsaturated double bonds" (Raab, 1972).

\section{Toxicity}

The toxicological effects of natamycin in this paper will be limited to oral doses. The acute toxicity of natamycin has been determined for mice, rats, guinea pigs, and rabbits through clinical studies with little agreement. The $\mathrm{LD}_{50}$ of natamycin after oral administration has been determined to be $1.5 \mathrm{~g} / \mathrm{kg}$ for mice and rats and $0.45 \mathrm{~g} / \mathrm{kg}$ for guinea pigs (Struyk et al., 1958). Levinskas et al. (1966) determined the $\mathrm{LD}_{50}$ as $2.7-4.7 \mathrm{~g} / \mathrm{kg}$ and $1.4 \mathrm{~g} / \mathrm{kg}$ for rats and rabbits, respectively. The intake of natamycin did not cause any gross lesions on the animals that died. It should be noted that irritation of the mucosa was seen in rabbits. Near lethal doses of natamycin caused growth inhibition, diarrhea, and edema (Struyk et al., 1958). 
Levinskas et al. (1966) conducted chronic toxicity tests for natamycin in rats and dogs. A two year study was performed for rats who were fed a $0.2 \%$ natamycin feed. This $1000 \mathrm{ppm}$ dosage of natamycin was well tolerated with only minor effects being observed. Observed effects included, slight decrease in food intake and slight inhibition in growth. Lactation and reproductions were normal for these animals over the two year test period. Results from chronic toxicity tests for dogs were similar to those for rats. Feed containing $0.05 \%$ and $0.025 \%$ natamycin, 500 and $250 \mathrm{ppm}$, were fed to dogs for over a two year period. Dogs subjected to 500 ppm dosages experience a slight increase in body weight. No observable effects occurred in dogs administered $250 \mathrm{ppm}$ dosages over the two year period. The acceptable daily intake (ADI) has been established at $0.30 \mathrm{mg} / \mathrm{kg}$ by the WHO and Food and Agriculture Organization of the United Nations (FAO). Approximately $0.002 \mathrm{mg} / \mathrm{kg}$ of natamycin is ingested by the average cheese consumer. This consumption equals $<1 \%$ of the established ADI (Oostendorp, 1981).

\section{Derivatives of Polyene Macrolide Antibiotics}

As previously mentioned, polyene macrolide antibiotics have characteristics such as extreme insolubility in water, severe instability, and serious parenteral toxicity. Many attempts have been made to overcome one or more of these problems by chemical modification of the chemical structure. Extensive research in the past 25 years has been conducted in polyene macrolide chemistry. Although some studies have been conducted on natamycin, the vast majority of research on this family of antibiotics have been performed for amphotericin B (Schaffner, 1987).

\section{Colloidal Dispersion Complexes}

The aqueous insolubility of polyene macrolide antibiotics is due to their intramolecular aggregation. "Introduction of detergent-like molecules, such as bile acids and fatty alcohol's sulfonates, or of polar polymers, such as polyborates, produce reversible complexes with 
increased molecular dispersion in aqueous medium" (Schaffner, 1987).

Bartner et al. (1958) prepared a neutral solution of amphotericin B in an aqueous solution of sodium desoxycholate. The preparation was lyophilized into a stable powder that can be reconstituted with water or dextrose solution. It was discovered that soluble amphotericin B prepared by the use of sodium desoxycholate does not enter into true solution (molecular dispersion). Although aqueous solutions of this preparation appear clear, they demonstrate the Tyndall effect, and exist in a fine colloidal dispersion of particles with dimensions smaller than those of bacteria (Bartner et al., 1958). Amphotericin B sodium desoxycholate complex (Fungizone ${ }^{\circledR}$ ) is manufactured by Bristol-Meyers Squibb Company and is the only polyene antibiotic complex approved by the Food and Drug Administration (FDA) for intravenous use (Bristol-Meyers Squibb Company, 1999).

An amphotericin B borate complex has also been produced (Vandeputte, 1973). The amphotericin B borate complex exhibits good water solubility, but only possess $80 \%$ the antifungal activity and has a significantly higher acute parenteral toxicity than that of the amphotericin B sodium desoxycholate complex (Schaffner, 1987).

\section{Semisynthetic Derivatives}

Chemical interactions with the zwitterionic carboxylate and the mycosamine of polyene macrolide antibiotics have produced a variety of semisynthetic antifungal derivatives with increased solubility as salts. Unlike colloidal dispersion complexes, these compound enter into molecular dispersion. Chemical interaction with free carboxylic acid and amino base functional groups is difficult. Water-soluble salts produced by direct interaction with strong acids or bases exhibit marked instability due to extremes of $\mathrm{pH}$ in aqueous solutions. Adjustments of solutions towards neutrality or the isoelectric $\mathrm{pH}$ of antibiotic results in precipitation due to colloidal aggregation and micelle formation. At acidic $\mathrm{pH}$, degradation occurs due to chromophore isomerization and glycosidic hydrolysis. At alkaline $\mathrm{pH}$, the macrocyclic lactone can be saponified with alterations of molecular structure and conformation resulting in total loss of biological activity. The conjugated polyene chromophore is also adversely affected by heat, 
oxidation, reduction, and ultraviolet radiation. Isomerization reactions, epoxide formation, or uptake of hydrogen produce structural alterations, which also lead to reduced or total loss of biological activity (Schaffner, 1987).

\section{Carboxyl Group Derivatives}

Mechlinski and Schaffner (1972) synthesized amphotericin B methyl ester.

Esterification was accomplished by bubbling diazomethane, prepared in tetrahydrofurane (THF), in a mixture of DMSO and amphotericin B. Amphotericin methyl ester demonstrated an equivalent biological activity to the parent antibiotic. Both compounds showed MIC values of 0.05-0.5 $\mu \mathrm{g} / \mathrm{ml}$ for Saccharomyces cerevisiae, Aspergillus niger, and Candida albicans (Mechlinski and Schaffner, 1972). Based on this study, it was concluded that esterification of the carboxyl group has little effect on the activity of the antibiotic.

The methyl ester derivative of amphotericin B was basic and its solubility was only marginally better than the parent. Schaffner and Mechlinski (1972) treated the methyl ester derivative of amphotericin B with hydrochloric acid to form a water soluble salt of this compound, amphotericin B methyl ester hydrochloride. This compound showed drastically improved water solubility and exhibited full antifungal activity. The solubility (molecular dispersion) of this compound was verified by UV spectroscopy. Amphotericin B methyl ester hydrochloride enters into true solution, unlike Fungizone®. E.R. Squibb and Sons, Inc. obtained the U.S. patent for amphotericin B methyl ester salts on August 9, 1977 (Sipos and Keseleski, 1977).

The production of methyl ester hydrochloride derivatives of nystatin, natamycin, mediocidin, candicidin, and trichomycin followed. All of these compounds exhibited good solubility and antifungal activity. For example, natamycin methyl ester hydrochloride has a solubility of $80.000 \mathrm{mg} / \mathrm{ml}$ in water at $25^{\circ} \mathrm{C}$ whereas the natamycin has a solubility of $0.052 \mathrm{mg} /$ $\mathrm{ml}$ for these same conditions (Schaffner and Mechlinski, 1972).

More comprehensive studies were performed examining the biological properties of polyene macrolide ester salts (Bonner et al., 1972; Bruzzese et al., 1975). In vitro studies 
against $S$. cerevisiae and in vivo studies against $C$. albicans in mice confirmed previous findings that polyene macrolide ester salts are at least as biologically active as their parent antibiotics and in some cases even more active(Bonner et al., 1972). Bruzzese et al. (1975) findings agreed with Bonner's, but also observed that the lengthening of the aliphatic chain in the ester group (methyl to butyl) produced a gradual decrease in activity. Bonner et al. (1972) also examined the acute intraperitoneal toxicities of polyene macrolides and their methyl ester hydrochlorides in mice. The methyl ester hydrochlorides were less toxic then their parent antibiotics for all polyenes tested.

Subsequent research focused on improved methods for preparation of methyl ester derivatives and extension of these methods to previously underivatized polyene antibiotics (Bruzzese and Ferrari, 1974; Pandey et al., 1977). Researchers from around the world challenged the efficacy and toxicity of amphotericin B methyl ester. Many in vitro, in vivo, and toxicity studies were published for amphotericin B.

Bannatyne and Cheung (1977) compared the susceptibility of 465 clinical isolates of $C$. albicans to amphotericin B and amphotericin B methyl ester. Results from the study showed that amphotericin B and amphotericin B methyl ester had comparable activity against half of the strains, but against the remainder of the strains the activity of amphotericin B methyl ester was slightly lower than that of amphotericin B. In another study, the in vitro antifungal activity of amphotericin B methyl ester was compared to the parent against a variety of pathogenic and potentially pathogenic fungi (Howarth et al., 1975). Test organisms used in this study included: Aspergillus fumigatus, A. niger, Blastomyces dermatitidis, C. albicans, C. guilliermondii, $C$. pseudotropicalis, Cladosporium sp., Coccidioides immitis, Cryptococcus neoformans, Histoplasma capsulatum, H. duboisii, Microsporum canis, M. gypseum, Muco pusillus, Oidiodendron kalrai, Paracoccidioides brasiliensis, Phialophora compactum, P. dermatitidis, P. verrucosa, Rhizopus arrhizus, Sepedonium sp., Sporothrix schenckii, Syncephalastrum, Torulopsis glabrata, Trichophyton memtagrophytes, T. rubrum, and T. simii. Amphotericin B methyl ester showed significant antifungal activity against most fungi, but was slightly less active then amphotericin B. The yeast cells of most fungi were killed at concentrations of 1 $\mu \mathrm{g} / \mathrm{ml}$ or less for either antibiotic. Filamentous forms of dimorphic fungi were more resistant 
requiring 10 to $50 \mu \mathrm{g} / \mathrm{ml}$ concentrations of these amphotericin B and amphotericin B methyl ester to cause death to these organisms (Howarth et al., 1975).

In vivo studies of amphotericin B (Fungizone ${ }^{\circledR}$ ) and amphotericin B methyl ester against experimental histoplasmosis, blastomycosis, cryptococcosis, and candidosis in mice were performed (Bonner et al., 1975). Mean effective doses $\left(\mathrm{ED}_{50}\right)$ for amphotericin $\mathrm{B}$ methyl ester were slightly higher for Histoplasma, Blastomyces, and Cryptococcus infections than those of the parent compound. In contrast, the $\mathrm{ED}_{50}$ for Candida infections for amphotericin $\mathrm{B}$ were slightly higher than that of amphotericin B methyl ester.

A similar in vivo study of amphotericin B (Fungizone $\left.{ }^{\circledR}\right)$ and amphotericin B methyl ester against $C$ albicans, $C$. neoformans, and $B$. dermatitidis in mice was performed (Gadebusch et al., 1976). Findings from these studies showed that amphotericin B methyl ester was drastically less efficacious then amphotericin B. Researchers of this study proposed that amphotericin B methyl ester has a lower avidity than amphotericin B for membrane-binding sites, is inactivated to a greater extent at these sites, and is poorly distributed when compared to the parent antibiotic.

In addition, the therapeutic efficacy of amphotericin B (Fungizone $®)$ and amphotericin B methyl ester delivered intraperitoneal or intravenous in mice infected with experimental murine coccidioidomycosis while monitoring toxicity (Lawrence and Hoeprich, 1976). Amphotericin B methyl ester was less effective than Fungizone ${ }^{\circledR}$ at low doses, but at higher doses was more effective than the parent antibiotic. At higher doses over 30 days, Fungizone ${ }^{\circledR}$ was lethal and/or nephrotoxic, whereas amphotericin B methyl ester was therapeutically effective and nontoxic to the kidneys. Keim et al. (1976) performed toxicological studies of amphotericin B methyl ester and amphotericin B in mice, rats, and dogs. It was found that the acute and subacute toxicity of amphotericin B methyl ester was much less than for the parent antibiotic. Amphotericin B methyl ester is 20 times less toxic than amphotericin B when administered as a single intravenous dose in mice. Intraperitoneal studies in rats and dogs showed the methyl ester derivative was one-forth and one-eighth as nephrotoxic as amphotericin B.

Results from these studies indicated that amphotericin B methyl ester could be an 
improved therapeutic agent for the treatment of systemic mycoses when compared to that of amphotericin B. Experimental in vitro and in vivo results showed that amphotericin B methyl ester derivatives were somewhat less effective against fungi and yeasts, but there significantly lower toxicity warranted their use. It was found that amphotericin B methyl ester could be safely administered to mice at concentrations several times greater than concentrations of Fungizone ${ }^{\circledR}$ that caused death (Lawrence and Hoeprich, 1976).

Monji et al. (1975) conducted an experiment to investigate the metabolic stability of amphotericin B methyl ester. Intravenous and intraperitoneal administration of radioactive amphotericin $\mathrm{B}{ }^{14} \mathrm{C}$-methyl ester in mice revealed no de-esterification of the antibiotic derivative. The most significant finding of this investigation was that there was no accumulation of radioactivity in the kidneys after administration of radiolabelled amphotericin B methyl ester. A similar study was performed for a non-human primate, Macaca mulatta (Lawrence et al., 1980). Results agreed with the previous study, amphotericin B methyl ester was metabolically stable and no de-esterification occurred.

Between 1975 and 1980, 50 patients suffering from mycoses caused by seven different kinds of fungi were treated with amphotericin B methyl ester by intravenous and intrathecal routes of administration. Doses administered were typically five times greater than those given with Fungizone®. Nephrotoxicity with these treatments were uncommon, but other more serious neurotoxic side-effects were evident. "In some of the patients receiving high doses for prolonged periods of time, neuropsychiatric symptoms such as confusion, disorientation, withdrawl, lethargy, depression, anorexia and more rarely stupor and coma became evident. Electroencephalo abnormalities were also evident" (Schaffner, 1987). Ellis et al. (1982) conducted histopathological examinations of the brains of patients who exhibited symptoms of neurological toxicity and who died of their fungal infections. The autopsy study showed diffuse leukoencephalopathy which consisted of proliferation of gemistocytic astrocytes, pallor of the myelin and accumulation of macrophage. These conditions were found in the cerebrum and cerebellar white matter (Ellis et al., 1982).

It was suggested (Hoeprich et al., 1982) and then later proved (Schaffner, 1987) that lots of amphotericin B methyl ester used in the treatment of patients revealed a complex of polyene 
macrolide components. In addition to monomethyl ester amphotericin B, the complex consisted of di-, tri-, tetra-, and penta-methylated amphotericins. Additional methylations occurred exclusively on the free amino group and the hydroxyl groups of the mycosamine moiety. None of the overmethylation involved the hydroxyl groups of the aglycone. It is believed that intermolecular bonding prevented this occurrence.

Amphotericin B amides have been produced by reaction of amines with the free carboxyl group of the polyene macrolide aglycone (Stefańska et al., 1980; Jarzebski et al., 1981). In vitro studies of amphotericin B amides demonstrated that these compounds have similar activity to that of the parent antibiotic, whereas some are virtually inactive. "The antifungal activity of the amide derivative appear to depend on the lipophilicity of the amid substituent. No in vivo chemotherapeutic data from animal studies have been performed" (Schaffner, 1987). Grzybowska and Borowski (1990) have published a paper on the preparation of polyene macrolide hydrazides, but no biological studies on these compounds have followed.

\section{Amino Group Derivatives}

Although most research of polyene macrolide semisynthetic derivatives has focused on ester derivatives, the first derivatization of macrolide antibiotics occurred in 1961 by successfully forming an $\mathrm{N}$-alkyl derivative of natamycin (Schaffner and Borowski, 1961; Lechevalier et al., 1961). It involved the treatment of amphotericin B with a large excess (50 moles) of acyl anhydride at room temperature for 1 hour. The yield of this procedure was 70$75 \%$ and the purity of the compound was poor. Mechlinski and Schaffner (1972) later improved

this procedure by conducting the acylation with acetic anhydride in an improved reaction medium for the antibiotic. The solution was a mixture of DMSO and methanol. It was found that the $N$-acylation of amphotericin B drastically reduced this compounds biological activity. The minimum inhibitory concentration (MIC) for $N$-acetyl amphotericin B, $N$-iodoacetyl amphotericin B, $N$-propionyl amphotericin B, and $N$-succinyl amphotericin B was 1.0 - 10.0 $\mu \mathrm{g} / \mathrm{ml}$ for test organisms $S$. cerevisiae, A. niger, and C. albicans compared to $0.05-0.5 \mu \mathrm{g} / \mathrm{ml}$ for the parent antibiotic (Mechlinski and Schaffner, 1972). 
Based on research from 1972, the development of semisynthetic polyene antibiotics derivatized at the free amino group did not occur. Researchers wrongfully hypothesized that an intact amino group on polyenes was required for biological activity. It was not until the early 1980's when Schering-Plough Corp. ( Bloomfield, NJ) began developing $N$-aminoacyl amphotericin B methyl ester derivatives that research in this area was revisited (Oblack et al., 1981).

Schering-Plough Corp. developed N-D-ornithyl amphotericin B methyl ester in 1980. Galgiani and VanWyck (1984) compared the efficacy of $N$-D-ornithyl amphotericin B methyl ester and Fungizone ${ }^{\circledR}$ against $C$. albicans infection in rats. At low doses, 0.1 and $0.5 \mathrm{mg} / \mathrm{kg}$, the drugs were equally effective in preventing deaths. At doses of $2.0 \mathrm{mg} / \mathrm{kg}$, mortality occurred in rats treated with Fungizone ${ }^{\circledR}$, whereas $N$-D-ornithyl amphotericin B methyl ester was effective and non-toxic. Graybill and Kaster (1984) showed that Aspergillus was equally susceptible to amphotericin B and $\mathrm{N}$-D-ornithyl amphotericin B methyl ester. These findings were supported by in vitro and in vivo tests in mice.

A comprehensive comparative in vitro and in vivo evaluation of $N$-D-ornithyl amphotericin B methyl ester, amphotericin B methyl ester, and amphotericin B was performed by researchers at Schering-Plough Corp. (Parmegiani et al., 1987). In vitro studies indicated that $N$-D-ornithyl amphotericin B methyl ester was more active than the other two drugs against Candida spp. and other fungi. In vivo, the dose response of $N$-D-ornithyl amphotericin B methyl ester was similar to amphotericin B to produce a 10,000-fold reduction of C. albicans in a mouse kidney infection and was much more effective then amphotericin B methyl ester. Acute intravenous $50 \%$ lethal doses in mice revealed that $N$-D-ornithyl amphotericin B methyl ester was one-ninth as toxic is amphotericin B, but twice as toxic as amphotericin B methyl ester.

Czerwiński et al. (1986) highlighted a general procedure for the preparation of $N$ dimethylaminoacyl derivatives of polyene macrolide antibiotics by combining the native polyene antibiotic in dimethylformamide (DMF) with an excess of $N$-hydroxysuccinimide ester in the presence of triethylamine. Czerwiński et al. (1991) later published a more comprehensive paper on the synthesis and biological properties of $N$-alkyl derivatives of amphotericin B. One particular $N$-alkyl derivative of amphotericin, $N$-(3'-N-dimethylaminosuccimido) amphotericin 
B, was of interest. $N$-( $3^{\prime}-N$-dimethylaminosuccimido) amphotericin B is achieved by the Michael addition reaction of amphotericin B with the $N$-substituted malemide $\left(N-\left(3^{\prime}-N-\right.\right.$ dimethylaminopropyl)malemide hydrochloride). MIC studies for $N-\left(3^{\prime}-N\right.$ dimethylaminosuccimido) amphotericin B showed it to possess equivalent activity to amphotericin B for test organisms S. cerevisiae, C. albicans, C. tropicalis, Geotrichum candidum, Torulopsis candida, and Trichophyton nanum. N-(3'-N-dimethylaminosuccimido) amphotericin B was less biologically active against C. arborea, C. mycoderma, and A. nidulans, but more active against A. niger, Penicillium cytrinum, and Mucor mucedo (Czerwiński et al., 1986). The uniqueness of $N$-(3'-N-dimethylaminosuccimido) amphotericin B is the presence of an additional, basic nitrogen atom in the modified aminosugar moiety.

\section{Mode of Antimicrobial Action}

The mode of action of polyene antibiotics mimics the hemolytic activity of saponins (Demel et al., 1965). Saponin, from an aqueous phase, penetrates and complexes with surfaceabsorbed complexes, particularly cholesterol. Cholesterol is removed from the cell membrane by a saponin solution. Extensive rearrangement of membrane lipids occurs and changes the state of the membrane. The membrane changes from a free flowing lamellar state to a micellar state due to the aggregation by the saponin-cholesterol complex. The micellar state resembles a hexagonal lattice. The hexagonal lattice structure is more permeable than the original membrane due to its pits and holes (Bangham and Horne, 1962; Lucy and Glauert, 1964).

Similarities between polyene antibiotics and saponins were realized after the effects of nystatin, filipin, and amphotericin B were examined on Saccharomyces and Candida strains. These studies indicated that losses of $\mathrm{K}^{+}, \mathrm{NH}_{4}{ }^{+}$, inorganic phosphate, carboxylic acids, sugar phosphates, nucleotides, and protein occur from the action of polyene antibiotics. Harsch and Lampen (1963) conducted a study examining the $\mathrm{K}^{+}$transport system of $S$. cerevisiae after exposure to the polyene antibiotic $N$-Acetylcandidin. At low levels, the binding of $N$ Acetylcandidin to the membrane reduces the efficiency of the ion transport into and out of the cell. The usual $\mathrm{Na}^{+}-\mathrm{K}^{+}$transport balance is adversely affected. This effect is reversed by the 
addition of $\mathrm{K}^{+}$and $\mathrm{NH}_{4}^{+}$and is known as sublysis.

$N$-Acetylcandidin, at high levels $(>100 \mu \mathrm{g} / \mathrm{ml})$, lysis the cell membrane by causing hexagonal pits. Loss of $\mathrm{NH}_{4}^{+}$occurs and blocks the cells ability to accumulate $\mathrm{K}^{+}$by depriving it of a necessary energy source. The addition of $\mathrm{K}^{+}$and $\mathrm{NH}_{4}{ }^{+}$did not cause this effect to be reversible. Loss in efficiency and the ability to accumulate $\mathrm{K}^{+}$by ion transport expedites cell death (Harsch and Lampen, 1963).

A similar study investigating intracellular loss of potassium in C. albicans after exposure to a number of polyene antibiotics was performed. Results from this study were less conclusive. Some antibiotics performed identical to $N$-Acetylcandidin where others did not. A few antibiotics caused irreversible membrane damage immediately at low concentrations. (Zygmunt, 1966). These results suggested that all polyene antibiotics do not have a similar mode of action.

Polyene macrolide antibiotics can be divided into two groups. Group 1 (46-63 carbon atoms) include: $N$-Acetylcandidin, nystatin, candidin, candicidin, and amphotericins A and B). These antibiotics have a specific effect on cation permeability at low concentrations. Their effects on glycolysis can be completely or partially reversed under appropriate conditions (Kinsky, 1967; Weissmann and Sessa, 1967). Group 2 (33-37 carbon atoms) include filipin, etruscomycin, and natamycin). Antibiotics in group 2 cause more extensive cell membrane damage than members of the group 1 because of their ability to interact with membrane phospholipids. Antibiotic-phospholipid interaction induces increased surface pressure to the sterol present in the membrane and causes reorientation of the cell membrane. Loss of vital cell constituents and osmotic shock occurs to the cell (Demel et al., 1968; Morris and Hart, 1978). The effects of group 2 polyene antibiotics cannot be reversed or prevented.

A relationship exists between the number of carbon atoms, size of macrolide ring, and the destructive power of the antibiotics to the cell membrane. Polyene antibiotics with smaller macrolide rings are more destructive to the cell membrane. No relationship for cell membrane damage exists for number of conjugated double bonds or net charge (Kinsky, 1967). It is important to note that relationships discussed are for relative degree of damage to the membrane and not on relative potency of the antibiotics in inhibiting growth. Heptaenes inhibit growth at 
lower concentrations than tetraenes.

In 1965, it was discovered that the presence of sterol in the cell membrane is necessary for polyene sensitivity by researchers at two institutions, independently, by similar methods. Mycoplasma laidlawii strain A, unlike most mycoplasmas, does not require sterols. When grown in the presence of cholesterol the organism incorporates the sterol into its membrane. Weber and Kinsky (1965) discovered, "The polyene antibiotic, filipin, inhibited growth and caused lysis of Mycoplasma laidlawii cells which had been cultured in the presence of cholesterol. The antibiotic did not inhibit growth and did not promote lysis of the organism when grown in the absence of cholesterol." Similarly, Feingold (1965) observed these same effects for Mycoplasma laidlawii when treated with amphotericin B.

The sterol hypothesis for the mode of action of polyene antibiotics was proven again by a unique study on the effects of polyene antibiotics on growth and sterol-induction of oospore formation by Pythium and Phytophthora species. Pythium and Phytophthora species, destructive plant pathogenic fungi, require an exogenous source of sterol for reproduction by sporangia or oospores (Hendrix and Lauder, 1966). Polyene antibiotics completely blocked oospore formation for Pythium periplocum when cholesterol was incorporated.

The interaction of polyenes with sterols was quantified by measuring changes in surface pressure occurring when polyenes were injected beneath monolayers of lipids or lipid mixtures at varying initial surface pressures. Changes occurred because interactions altered the spatial orientation of the lipid molecules with respect to the air-water interface. This research allowed scientists to quantify and compare the relative activity of various polyenes with numerous sterols (Hamilton-Miller, 1974).

Numerous in vitro studies were performed with polyene antibiotics on synthetic lecithincholesterol bilayers to better understand the biological properties and selective membrane toxicity of polyene antibiotics. Results from these studies lead to the following conclusions: (1) polyene-induced permeability alteration in membrane systems is affected by the composition of membrane phospholipid fatty acyl chains; (2) the selective toxicity of polyenes is related to the distribution of double bonds in the sterol nucleus; (3) polyenes differ in membrane selectivity (Zutphen et al., 1971; Norman et al., 1972a; Norman et al., 1972b; Hsuchen and Feingold, 
1973).

Brajtburg et al. (1990) also reviewed the molecular basis of sterol specificity of polyenes. Spectrophotometry has demonstrated that polyene antibiotics bind more avidly to ergosterol than to cholesterol and to ergosterol-containing membranes than to cholesterol-containing membranes. Two theories have been presented to explain this phenomenon. One theory suggests that polyene antibiotics and sterols, with the participation of $\mathrm{H}_{2} \mathrm{O}$, form a "cage" linking the sterol between the carboxyl and amino end of the molecule by hydrogen bonds. Functional groups involved in the hydrogen bonds are the hydroxyl groups of the sterol and the carboxyl group. The "cage" is completed by participation of the free amino group. Both ergosterol and cholesterol are 3- $\beta$-hydroxy sterols, and it can be assumed that their reactions with polyenes involving hydrogen bonds are equivalent (Brajtburg et al., 1990).

Another theory to help explain the sterol specificity involves the involvement of van der Waals forces (non-specific forces). Ergosterol contains an double bond at C-22 on its alkyl side chain whereas cholesterol does not contain this double bond. The double bond of ergosterol limits the number of possible conformations this molecule can obtain. The predominant confirmation of ergosterol is flat in shape. Cholesterol with no double bond is more flexible and can exist in numerous different shapes, one being flat. The flat shape of the ergosterol molecule may facilitate intermolecular contacts with the polyene macrolide. This theory helps better explain the specificity of polyene antibiotics for ergosterol, whose conformational state favors interaction (Brajtburg et al., 1990).

Chéron et al. (1988) conducted a quantitative structure-activity relationship study of amphotericin B and sixteen semisynthetic derivatives obtained by modification at the carboxyl and amino groups. Results from this study indicate: (1) the presence of positively charged nitrogen atom is indispensable for biological activity and antibiotic-sterol interaction and (2) the lack of free carboxyl group in the molecule favors the differentiation between cholesterol and ergosterol containing cells. 


\section{Resistance and Tolerance}

There has never been a resistance problem with polyene antibiotics in practice since their introduction in the 1950's. Laboratory experiments have shown that resistance to polyenes can occur by sensitive organisms by compositional alteration of the cell membrane and resistance to oxidative damage. However theoretically and experimentally achievable, resistance to polyene antibiotics is of no clinical importance.

The sensitivity to natamycin of molds and yeasts isolated in cheese warehouses where natamycin has been used for various periods was determined (De Boer and Stolk-Horsthuis, 1977). Strains of molds isolated in cheese warehouses showed no decrease in sensitivity to natamycin after numerous transfers of the culture was subjected to sub-lethal doses of the antibiotic. No differences were found in natamycin sensitivity between fungal populations for warehouses utilizing natamycin for 10 years and ones that never introduced the antimycotic. A similar study was performed in factories producing dry sausage. No differences were found in natamycin sensitivity for mold and yeast isolates in factories using natamycin and ones that never used the preservative (De Boer et al., 1979).

The Joint FAO/WHO Expert Committee on Food Additives (1976) addressed concerns in using natamycin, a therapeutic agent, in food. Objections to the use of therapeutic antibiotics in food presented to the committee were based on the following factors.

1. There is a rapid appearance of high levels of acquired resistance in intestinal bacteria when antibiotics (especially tetracyclines or streptomycin) are ingested. The levels may become so high, indeed, that the greater concentrations used therapeutically cease to be effective.

2. Resistance to one antibiotic of a group (e.g. tetracyclines) is accompanied by "cross-resistance" to others of that group.

3. There is a simultaneous transfer of resistance to antibiotics and drugs unrelated to the one used to induce the resistance.

4. There is a ready transfer of resistance from harmless to pathogenic Gramnegative bacteria, diminishing the therapeutic value of the antibiotic. 
The objections stated only occur minimally with natamycin or not at all. Natamycin has no effect on bacteria and is not used to control them. Clinical resistance to natamycin for fungi and yeasts has never occurred. Laboratory experiments have achieved organisms with minor resistance to natamycin, but they are of no clinical importance. In addition, resistance among fungi and yeasts were achieved by selection and not by induction. The same is true for related polyenes.

Cross-resistance between polyene antibiotics is infrequent. In particular, natamycinresistant strains in vitro has never achieved cross-resistance with other polyene antibiotics. Transferable resistance for yeasts and fungi can not occur because DNA cannot be transferred across the cell-wall membrane barrier as it is in bacterial cells.

Studies investigating acquired-resistance to polyene antibiotics focused on C. albicans. These studies indicated that polyene resistance is associated with altered sterol patterns (Athar and Winner, 1971; Hamilton-Miller, 1972; Subden et al., 1977; Hitchcock et al., 1987). In vitro studies showed the sterol metabolism of polyene-resistant mutant $C$. albicans was quantitatively and qualitatively different then sensitive parent strains. It is believed that sterol patterns are altered by changes in biosynthesis of lanosterol to ergosterol. Mutants lose the ability to demethylate C-14 methyl groups by oxidation in the biosynthesis of ergosterol. A primitive biogenetic precursor, 14- $\alpha$-methyl sterol, is incorporated in place of ergosterol in the cell membrane (Dekker and Gielink, 1979; Ziogas et al., 1983). All but one study, indicate a decrease in ergosterol content for resistant C. albicans. Hamilton-Miller (1972) showed a two to three time increase in ergosterol. In both cases, polyene-resistant mutants have protected their cell membrane by substituting ergosterol with sterols that bind polyenes less effectively or by adding sacrificial ergosterol for polyene binding. Resistance to polyene antibiotics may be due to a single gene mutation. Woods (1971) showed this effect for nystatin resistance in yeasts and it was later shown for natamycin resistance in A. nidulans (Dekker and Gielink, 1979).

Two related theories are offered to explain the mechanism of resistance for polyene antibiotics. Pierce et al. (1978) proposes that sterol esters incorporated into the membrane would increase resistance to polyenes. Sterol esters have a lower affinity for polyenes then do 
free sterols. Sokol-Anderson et al. (1988) believe that resistance of cells to oxidative damage may be another factor affecting polyene antibiotic sensitivity. These theories are related to the primitive biogenic precursor theory. Resistance to oxidation may cause fungi and yeasts to loss the ability to demethylate $\mathrm{C}-14$ methyl groups. Incorporation of methyl sterols into the membrane will decrease the affinity for polyene antibiotics to bind to the cell membrane.

It should be noted that resistant fungi and yeast cultures showed decreased growth rate, reduced production of germ tubes, slower production of chlamydospores, reduced suspension stability, and reduced pathogenicity when compared to sensitive parent strains (Athar and Winner, 1971). " The quantitative and qualitative alterations in the sterols of the fungal membrane responsible for reduced affinity to polyene antibiotics, seem at the same time to lower the fitness of mutants which are resistant to these antibiotics" (Dekker and Gielink, 1979). If mutation for resistance would occur in clinical conditions it is improbable or impossible that these organisms would be able to establish appreciable numbers.

\section{Medical Applications}

Polyene antibiotics are widely used to treat superficial and systemic mycoses. Amphotericin B, nystatin, and natamycin are the most useful clinically of all the polyene antibiotics. Polyene antibiotics are ideal for treating fungal infections of the skin because of their broad antifungal spectrum, high efficacy, good penetration, no metabolism, low percutaneous absorption, and lack of systemic toxicity (Stock, 1981). Polyene antibiotics are prescribed to treat superficial infections caused by Epidermophyton spp., Trichophyton spp., Microsporum spp., and C. albicans. Deep mycoses such as systemic candidiasis, cryptococcosis, and systemic aspergillosis are also treated with polyene antibiotics (Purdue Research Foundation, 1998).

Since 1980, more specific antibiotics for yeast and mold infections have been developed and are being utilized. This is especially true for Candida infections of skin or mucous membranes (oral, genital, and respiratory). Antiseptic and disinfectant preparations containing 
phenols, fatty acids, benzoic and salicylic acid, quaternary ammonium compounds, hydroxychinolines, and triphenylmethandyes are used to treat superficial fungal infections (Stock, 1981).

Intravenous administration of amphotericin B is the most effective agent for the treatment of many systemic fungal infections (Keim et al., 1973). Administration of amphotericin B intravenously is effective, but its use is limited to its poor solubility in most vehicle solutions and because of its high relative nephrotoxicity. Coccidionidomycosis, the fungal infection of Coccidioides immitis, more commonly known as meningitis, is a serious chronic disease that requires amphotericin B for its treatment (Lawrence and Hoeprich, 1976). No other therapeutic agent is available to treat this condition.

The only medical application of natamycin being used in the U.S. is for treatment of fungal infections of the eye. Alcon Laboratories, Inc. (Fort Worth, TX) manufactures eye-drops under the trade name, Natacyn $A \circledast$. Natacyn $A \circledast$ is a preparation of a $5 \%$ solution of natamycin that is used to treat fungal keratistis and allergy irritation of the eye (Alcon Laboratories, Inc., 1999). Natamycin is the only FDA approved ocular antifungal agent in the U.S. (Primary Care Optometry News, 1999).

A conversation with Dr. Carl P. Schaffner, Professor of Microbiology at Rutgers University, notified me that their has been a resurgence in research concerning polyene antibiotics and their derivatives (Schaffner, 1998). Dr. Schaffner and colleagues were responsible for establishing the chemical structure of amphotericin B by X-ray crystallography and was the first to experiment with semisynthetic derivatives of polyene antibiotics in the 1960 's.

Research has been stimulated in this area due to increased incidence of systemic mycoses, especially meningitis. Opportunistic pathogens, yeasts and molds, which are normally non-problematic in healthy people, may become pathogenic in particular in patients with impaired defense mechanisms. The immunocompromised population of the U.S. is increasing every year by the growth in numbers of the elderly and people suffering from the HIV virus. Treatment of systemic fungal infection by intravenous amphotericin B for AIDS patients is common. Preliminary investigations has shown that this treatment has also made T-cells more 
resistant to the HIV virus. The use of polyene antibiotics to treat HIV infected individuals is being studied. Dr. Schaffner also reported that the U.S. Army Corp. is investigating additional applications of polyene antibiotics to treat numerous tropical diseases.

\section{Food Applications}

A good preservative agent satisfies the following requirements, (1) it is active against spoilage organisms that can cause deterioration; (2) it must remain active under normal conditions throughout the products shelf-life; (3) it is safe for human consumption; (4) it must not increase the cost of the product appreciably; and (5) it must not affect the quality, appearance, smell, color, or flavor of the product. Natamycin satisfies these general requirements, but yet its usage as a food preservative is limited due to how it is regulated by the FDA and the European Union (EU).

The potential uses of polyene antibiotics for the preservation of food was recognized soon after their discovery. Klis et al. (1959) reported that a foliage spray of griseofulvin controlled Botrytis cinera infections of lettuce and Alternaria solani infections of tomatoes. He proposed to use polyene antibiotics to control the fungal spoilage of fresh fruits and vegetables as well as dehydrated, smoked, and salted fish and meats. Amphotericin B and candicidin were experimentally introduced into refrigerated apple juice to extend the shelf-life of this product (Do and Salunkhe, 1964). Recently, the FDA discovered two juice manufacturers incorporating natamycin in orange juice to extend the shelf-life of that product (Perry, 1998).

Although natamycin is useful in inhibiting fungi and yeast growth for fresh fruits and vegetables, dried and salted meats, and beverages, its use for those product is illegal. The only food application of natamycin allowed in the U.S. is for the treatment of block and sliced cheeses. Typically, a 0.05 to $0.25 \%$ aqueous suspension of natamycin is applied to the surface of cheese by spraying or dipping. Natamycin only penetrates the surface of cheese slightly, concentrating on the surface, because of its insolubility in water (Luck and Jager, 1997). In addition to the use of natamycin on cheeses, the EU also allows natamycin to be used as a surface growth inhibitor for dried and cured sausages. 


\section{Regulatory Status}

The FDA classifies natamycin as a food additive permitted for direct addition to food for human consumption (CFR, 1998a). The Code of Federal Regulations (CFR) specifies strict usage and quality standards for natamycin. The CFR requires natamycin's purity to be $97 \% \pm$ $2 \%$ on an anhydrous basis, not contain more than $1 \mathrm{ppm}$ arsenic, not contain more than $20 \mathrm{ppm}$ heavy metals (as $\mathrm{Pb})(\mathrm{CFR}, 1998 \mathrm{a})$. Application and usage levels of natamycin according to the CFR, "The additive may be applied to the surface of cuts and slices of cheese to inhibit mold spoilage with the following limitations: (1) the additive may be applied by dipping or spraying using an aqueous solution containing 200 to $300 \mathrm{ppm}$ of the additive; (2) the additive may be applied to the surface of those cuts and slices of cheese(s) listed in part 133 of this chapter only if the cheese standards provide for the use of "safe and suitable" mold-inhibiting ingredients" (CFR, 1998a). Cheese varieties in which a safe and suitable antimycotic agent(s) can be applied to the cheese surface listed in part 133 of the CFR include: asiago fresh and asiago soft cheese, asiago medium cheese, blue cheese, brick cheese, caciocavallo siciliano cheese, cheddar cheese, edam cheese, gorgonzola cheese, gouda cheese, granular and stirred curd cheese, grated cheeses, gruyere cheese, hard cheeses, monterey cheese and monterey jack cheese, mozzarella cheese and scamorza cheese, muenster and munster cheese, parmesan and reggiano cheese, provolone cheese, romano cheese, samsoe cheese, and swiss and emmentaler cheese (CFR, 1998b).

Natamycin is regulated by the EU differently then how it is regulated by the FDA. The European Parliament and Council Directive (95/2/EC) of February 20, 1995 on food additives other than colors and sweeteners allows natamycin to be used as a surface treatment to prevent mold growth on hard, semi-hard and semi-soft cheeses, and dried, cure sausages. Maximum level allowed on the food surface is $1 \mathrm{mg} / \mathrm{dm}^{2}$ (Jukes, 1998). The EU has designated a numbering system for all food additives. Natamycin's designation is E235. The numbering system is being adapted for international use by the Codex Alimentarius Commission who are developing an International Numbering System (INS).

The EU specifies the maximal level of natamycin on the cheese surface, $1 \mathrm{mg} / \mathrm{dm}^{2}$. 
Conversely, the FDA only regulates the concentration at which natamycin may be applied, 200 to $300 \mathrm{ppm}$ aqueous solution. The maximum level of natamycin on the cheese surface in the U.S. is not regulated. This loophole in the regulation promotes processors to use higher levels of natamycin than would be achieved by one application of a 200 to 300 ppm aqueous solution.

The CFR also specifies the way in which natamycin is applied, dipping or spraying in an aqueous solution. The insoluble nature of natamycin in water makes application difficult. Constant clogging of spray nozzles and errors in preparing natamycin aqueous solutions results in inappropriate application. On December 1, 1998 the FDA amended the food additive regulations to provide for the safe use of a dry form of natamycin as an antimycotic in cheeses (FR, 1998). The action was in response to a petition filed by Protein Technologies International, Inc. (St. Louis, MO).

Requirements for certification are required for natamycin for medical application. The CFR dictates standards of identity, strength, quality and purity for a natamycin ophthalmic suspension, the only specified application (CFR, 1998c). Part 436 of the CFR details tests and methods of assay of antibiotic and antibiotic-containing drugs.

\section{Cheese Spoilage Organisms}

Bullerman (1981) reported that $82 \%$ of mold isolates on refrigerated Cheddar cheese belongs to the genus Penicillium, 7\% Aspergillus, 1\% Fusarium, 1\% Alternaria, and the remaining $9 \%$ were distributed over several other genera. In another study, the most frequent spoilage molds on refrigerated cheeses included; Penicillium 61\%, Aspergillus $12 \%$, Cladosporium 9\%, and Phycomycetes 13\% (Jarvis, 1983). Jarvis (1983) reported the most common Penicillium species isolated in cheese samples were $P$. roqueforti, $P$. griseofulvum, $P$. chrysogenum, P. expansum, P. cyclopium, P. crustosum, P. citrinum, P. aurantiogriseum, P. brevi-compactum, and $P$. viridicatum.

The most comprehensive study of cheese spoilage organisms to date was performed by F. Lund and colleagues in 1994. In this study, 371 fungal isolates were identified in hard, semihard, and semi-soft cheeses from Denmark, France, Greece, UK, and other countries. 
Penicillium sp. accounted for $91 \%$ of the fungal isolates (Lund et al., 1995). Most of the isolates $(88 \%)$ found on the cheese belonged to the following species; $P$. commune, $P$. nalgiovense, $P$. verrucosum, $P$. solitum, $P$. roqueforti, Aspergillus versicolor, $P$. crustosum, $P$. atramentosum, P. chrysogenum, and P. echinulatum (Lund et al., 1995).

Lund et. al. (1995) reported that $P$. commune, $P$. verrucosum, $P$. solitum, P. roqueforti, and $P$. nalgiovense accounted for most of the mold isolates in hard (e.g. Cheddar) vacuum packaged cheese. Inadequately vacuum-packed cheese showed a prevalence of $P$. roqueforti (Lund et al., 1996). Contamination of cheese by $P$. roqueforti and $P$. commune can be attributed to their prevalence in cheese warehouses and ripening rooms. Penicillium roqueforti and $P$. commune are ripening molds commonly used to promote specific flavor and color characteristics for several cheese varieties.

The production of mycotoxins by cheese spoilage organisms is a food safety concern. Ochratoxin is a mycotoxin produced by species in the genera Aspergillus and Penicillium, two common cheese spoilage organisms. The primary and most toxic form of ochratoxin is ochratoxin A (OA). Ochratoxin is commonly produced by $P$. verrucosum and A. ochraceous. OA production has been reported by A. ostianus, A. quercins (A. melleus), and A. sulphureus (A. fresenii) (Marquardt and Frohlich, 1992). The production of ochratoxin by Penicillium species is a subject of much controversy. The following Penicillium species are a few of those that have been reported in literature as producing ochratoxin: $P$. commune, $P$. chrysogenum, $P$. cyclopium, $P$. palitans, $P$. purpurescens, $P$. variable, $P$. verrucosum and $P$. viridicatum (Pohlmeier and Bullerman, 1978; Bullerman, 1980; Northolt et al., 1979; Marquardt and Frohlich, 1990). However, Pitt (1987) proved that P. viridicatum does not produce ochratoxin A as was widely reported. Penicillium verrucosum was the only species found to reliably produce OA in the subgenus Penicillium. This study found 48 of 84 isolates of $P$. verrucosum were capable of producing OA, with some isolates additionally able to produce citrinin. It is believed that earlier reports of OA production were due to misidentification of the fungi especially in the case of P. viridicatum (Pitt, 1987; Pitt and Hocking 1997).

The natural occurrence of OA in cheese has not been extensively surveyed, but instead most research has focused on isolating fungi contaminating cheeses and identifying their 
capability of producing mycotoxins in culturing media. In a 1976 study of moldy cheese trimmings, Bullerman found one cheese sample positive for the presence of OA. In a study by Pohlmeier and Bullerman (1978), a Cheddar cheese isolate (Penicillium sp.) was able to produce $\mathrm{OA}$ on Cheddar at 5,12 and $25^{\circ} \mathrm{C}$. Migration of the mycotoxin in the cheese was seen up to 7 $\mathrm{mm}$ from the fungus. A survey of Cheddar cheeses by Bullerman and Olivigni (1974) found $82.2 \%$ of fungal isolates were Penicillium followed by 6.6\% Aspergillus. Of the Penicillium species the researchers found $29.2 \%$ to be toxic to chicken embryos, with two isolates producing OA. Penicillium has been found to be the most common spoilage causing fungi in a variety of cheeses (Bullerman, 1976; Bullerman, 1981). Bullerman (1980) found 86\% of all domestic cheese and $80 \%$ of all imported cheeses to contain Penicillium as the major mycoflora. A. ochraceous was isolated from both domestic and imported cheeses at $0.8 \%$ and $0.2 \%$, respectively. This study also found $4.7 \%$ of domestic cheese and $2.1 \%$ of imported cheese isolates were capable of producing OA on culture media. In a study of visibly molded cheeses in The Netherlands by Northolt et al. (1980), P. verrucosum was frequently isolated and $A$. ochraceous was found in one sample. Sinha and Ranjan (1991) isolated A. ochraceous as the dominant spoilage organism in molded Bhutanese cheese. They additionally tested the cheese for mycotoxins and found five of nineteen to contain OA at levels ranging from $42 \mathrm{mg} / \mathrm{kg}$ to 116 $\mathrm{mg} / \mathrm{kg}$. Hocking and Faedo (1992) isolated P. verrucosum as one of the causes of thread mold spoilage in Cheddar cheese vacuum packaged for aging. Lund et al. (1995) found $P$.

verrucosum to be a frequent contaminant of a variety of cheeses from different countries. All $P$. verrucosum isolates were found to produce OA on culture media in this study.

Natamycin has been shown to delay mold growth on Gouda and Cheddar cheese during aging (Lück and Cheeseman,1978). Shahani et al. (1977) studied the effect of natamycin on A. parasiticus and $P$. patulum, producers of aflatoxin and patulin. The study showed that natamycin was effective in inhibiting growth on blocks of Cheddar and Parmesan cheese. The cheeses were tested for patulin and aflatoxin, but none was detected. Shahani et al. (1973) found that natamycin was able to retard growth of A. flavus, A. ochraceous, P. cyclopium and $P$. patulum on a variety of foods. This study found that at $1.0 \mathrm{ppm}$ of natamycin inhibited mycotoxin production from 25 to $97 \%$. 
Figures

Figure 1: Chemical structure of natamycin

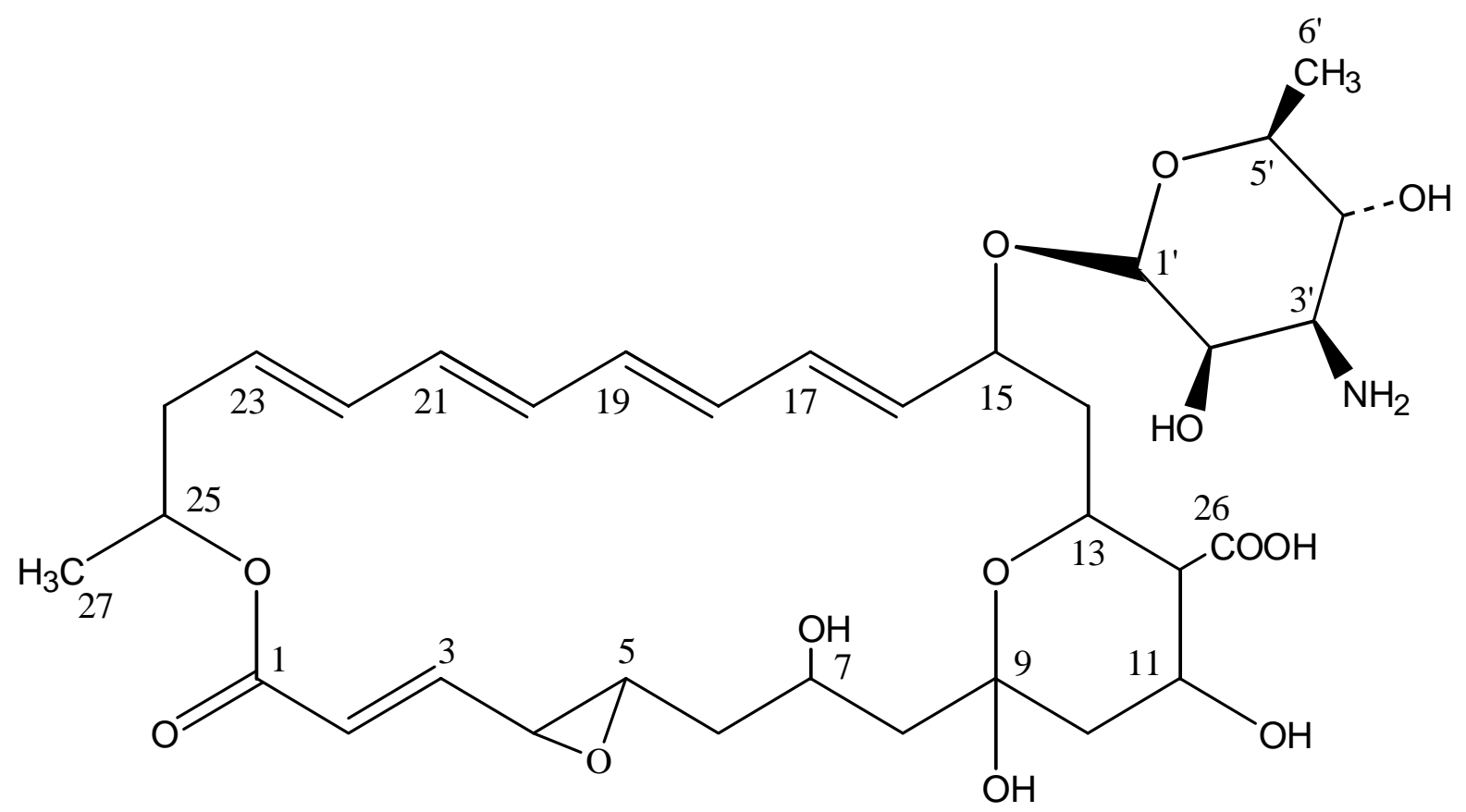


Figure 2: Natamycin crystals (dissolved in vegetable oil at 500X magnification)

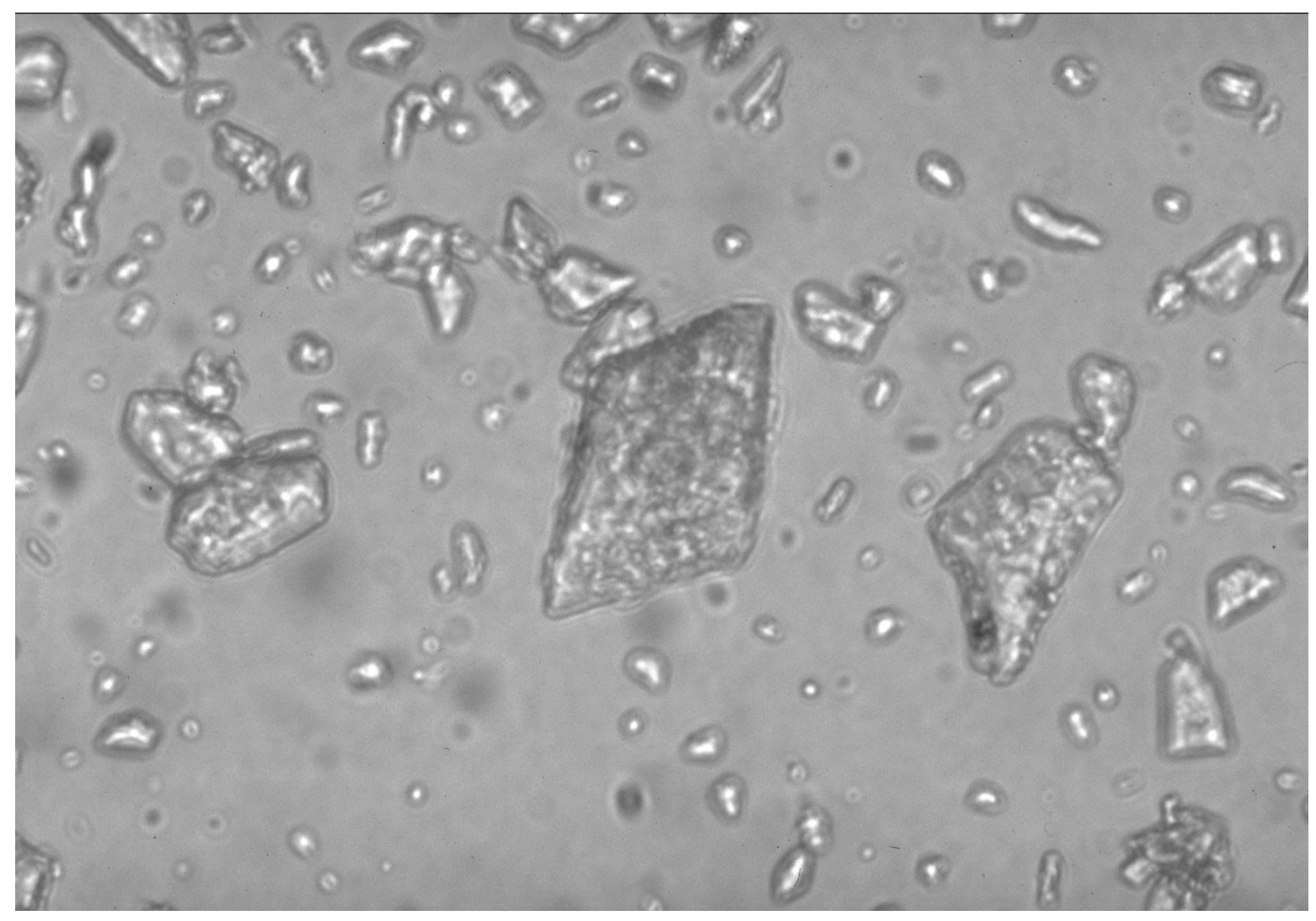




\section{References}

Alcon Laboratories, Inc. 26 February 1999. "Naphcon A". http://www.alconlabs.com/us.

American Cyanamid Company. 1960. New antifungal substance, pimaricin, and method of producing same. British Patent 846,933.

Athar, M.A. and Winner, H.I. 1971. The development of resistance by Candida species to polyene antibiotics in vitro. J. Med. Microbiol. 4:505-517.

Bangham, A.D. and Horne, R.W. 1962. Action of saponin on biological cell membranes. Nature. 196:952-955.

Bannatyne, R.M. and Cheung, R. 1977. Comparative susceptibility of Candida albicans to amphotericin B and amphotericin B methyl ester. Antimicrob. Ag. Chemother. 12:449-450.

Barr, F.S. 1959. Tennecetin, a new antifungal antibiotic. Toxicological studies. Antib. And Chemother. 9:406-410

Bartner, E., Zinnes, H., Moe, R.A. and Kulesza, J.S.. 1958. Studies on a new solubilized preparation of amphotericin B. In Antibiotics Annual 1957-1958. Medical Encyclopedia Inc., New York. 53-58.

Bonner, D.P., Mechlinski, W., and Schaffner, C.P. 1972. Polyene macrolide derivatives. III. Biological properties of polyene macrolide ester salts. J. Antibiotics. 25:261-262.

Bonner, D.P., Tewari, R.P., Solotorovsky, M., Mechlinski, W, and Schaffner, C.P. 1975. Comparative chemotherapeutic activity of amphotericin B and amphotericin B methyl ester. Antimicrob. Ag. Chemother. 7:724-729 
Brajtburg, J., Powderly, W.G., Kobayashi, G.S., and Medoff, G. 1990. Amphotericin B: Current understanding of mechanisms of action. Antimicrob. Ag. Chemother. 34:183-188.

Brik, H. 1981. Natamycin. Ch, In Analytical Profiles of Drug Substances, Vol. 10. K. Florey (Ed.), p. 513-561. Academic Press, Inc. New York.

Bristol-Meyers Squibb Company. 6 March 1999. “Fungizone (Amphotericin B for injection) intravenous". http://www.bms.com/products/index.html.

Bruzzese, T. and Ferrari, R. 1974. A new polyenic antibiotic. British Patent 1,359,473.

Bruzzese, T., Cambieri, M., and Recusani, F. 1975. Synthesis and biological properties of alkyl esters of polyene antibiotics. J. Pharm. Sci. 64:462-463.

Bullerman, L.B. and Olivigni, F.J. 1974. Mycotoxin producing-potential of molds isolated from Cheddar cheese. J. Food Sci. 39:1166-1168.

Bullerman, L.B. 1976. Toxigenic potential of molds isolated from moldy cheese trimmings. In Abstracts of Papers and Presented at the Sixty-third Annual Meeting of IAMFES. J. of Milk and Food Technol. 9:705.

Bullerman, L.B. 1980. Incidence of mycotoxic molds in domestic and imported cheeses. J. Food Safety. 2:47-58.

Bullerman, L.B. 1981. Public health significance of molds and mycotoxins in fermented dairy products. J. Dairy Sci. 64:2439-2452.

CFR. 1998a. Code of Federal Regulations. Title 21. Part 172. U.S. Government Printing Office, Washington, DC. 
CFR 1998b. Code of Federal Regulations. Title 21. Part 133. U.S. Government Printing Office, Washington, DC.

CFR. 1998c. Code of Federal Regulations. Title 21. Part 449. U.S. Government Printing Office, Washington, DC.

Chéron, M., Cybulska, B., Mazerski, J., Grzybowska, J., Czerwiński, A., and Borowski, E. 1988. Quantitative structure-activity relationships in amphotericin B derivatives. Biochem. Pharmac. 37:827-836.

Clark, W.L., Shirk, R.J. and Kline, E.F. 1964. Pimaricin, a new food fungistat. Fourth International Symposium on Food Microbiology, Göteborg, Sweden, June 1-5.

Czerwiński, A., Grzybowska, J., Borowski, E. 1986. N-Dimethylaminoacyl derivatives of polyene macrolide antibiotics. J. Antibiotics. 39:1025-1027.

Czerwiński, A., König, A., Zieniawa, T., Sowiński, P., Sinnwell, V., Milewski, S., and Borowski, E. 1991. New $N$-alkyl derivatives of amphotericin B synthesis and biological properties. J. Antibiotics. 44:979-984.

De Boer, E. and Stolk-Horsthuis, M. 1977. Sensitivity to natamycin (pimaricin) of fungi isolated in cheese warehouses. J. Food Prot. 40:533-536.

De Boer, E., Labots, H., Stolk-Horsthuis, M.., and Visser, J.N. 1979. Sensitivity to natamycin of fungi in factories producing dry sausage. Fleischwirtsch. 59:1868-1869.

Dekker, J. and Ark, P.A. 1959. Protection of the antibiotic pimaricin from oxidation and ultraviolet light by chlorophyllin and other compounds. Antib. And Chemother. 9:327-332. 
Dekker, J. and Gielink, A.J. 1979. Acquired resistance to pimaricin in Cladosporium cucumerinum and Fusarium oxysporum f.sp. narcissi associated with decreased virulence. Neth. J. Pl. Path. 85:67-73.

Demel, R.A., Van Deenen, L.L.M. 1965. Penetration of lipid monolayers by polyene antibiotics. J. Biol. Chem. 240:2749-2753.

Demel, R.A., Crombag, F.J.L., Van Deenen, L.L.M., and Kinsky, S.C. 1968. Interaction of polyene antibiotics with single and mixed lipid monomolecular layers. Biochim. Biophys. Acta. 150:1-14.

Do, J.Y. and Salunkhe, D.K. 1964. Effects of chemical treatments on refrigerated apple juice. Food Technol. 18:182-184.

Egmond, H.P. van. 1989. Mycotoxigenic fungal contaminants of cheese and other dairy products. Ch. 7, In Mycotoxins in Dairy Products. H.P. Van Egmond (Ed.), Elsevier Applied Science, New York.

Eliot, S.C., Vuillemard, J.C., and Emond, J.P. 1998. Stability of shredded Mozzarella cheese under modified atmospheres. J. Food Sci. 63:1075-1080.

Ellis, W.G., Sobel, R.A., and Nielsen, S.D. 1982. Leukoencephalopathy in patients treated with amphotericin B methyl ester. J. Infect. Dis. 146:125-137.

Feingold, D.S. 1965. The action of amphotericin B on Mycoplasma laidlawii. Biochem. Biophys. Res. Commun. 19:261-267.

FR 1998. Federal Register. Vol. 63. p. 66,014. U.S. Government Printing Office, Washington, DC. 
Galgiani, J.N. and VanWyck, D.B. 1984. Ornithyl amphotericin methyl ester treatment of experimental Candidiasis in rats. Antimicrob. Ag. Chemother. 26:108-109.

Gadebusch, H.H., Pansy, F., Klepner, C., and Schwind, R. 1976. Amphotericin B and amphotericin B methyl ester ascorbate. I. Chemotherapeutic activity against Candida albicans, Cryptococcus neoformans, and Blastomyces dermatitidis in mice. J. Infect. Dis. 134:423-427.

Graybill, J.R. and Kaster, S.B. 1984. Experimental murine Aspergillus. Comparison of amphotericin B and a new polyene antifungal drug, SCH 28191. Am. Rev. Respir. Dis. 129:292-295.

Grzybowska, J. and Borowski, B. 1990. Hydrazides - A novel type of derivatives of polyene macrolide antifungal antibiotics. J. Antibiotics. 43:907-908.

Hamilton-Miller, J.M.T. 1972. Sterols from polyene-resistant mutants of Candida albicans. J. Gen. Microbiol. 73:201-203.

Hamilton-Miller, J.M.T. 1974. Fungal sterols and the mode of action of the polyene antibiotics. Adv. Appl. Microbiol. 17:109-134.

Harsch, M. and Lampen, J.O. 1963. Modification of $\mathrm{K}^{+}$transport in yeast by the polyene antifungal antibiotic N-acetylcandidin. Biochem. Pharm. 12:875-883.

Hitchcock, C.A., Barrett-Bee, K.J., and Russell, N.J.1987. The lipid composition and permeability to azole of an azole- and polyene-resistant mutant of Candida albicans. J. Med. Vet. Mycology. 25:29-37.

Hendrix, J.W. and Lauder, D.K. 1966. Effect of polyene antibiotics on growth and sterolinduction of oospore formation by Pythium periplocum. J. Gen. Microbiol. 44:115-120. 
Hocking, A.D. and Faedo, M. 1992. Fungi causing thread mould spoilage of vacuum packaged Cheddar cheese during maturation. International J. of Food Microbiol. 16:123-130.

Hoeprich, P.D. 1982. Editorial: Amphotericin B methyl ester and leukoencephalopathy: The other side of the coin. J. Infect. Dis. 146:173-176.

Hoogerheide, J.C. 1961 and 1964. Unpublished Data, Quoted by Raab 1972.

Howarth, W.R.,Tewari, R.P., and Solotorovsk, M.. 1975. Comparative in vitro antifungal activity of amphotericin B and amphotericin B methyl ester. Antimicrob. Ag. Chemother. 7:5863.

Hsuchen, C. and Feingold, D.S. 1973. Selective membrane toxicity of the polyene antibiotics: Studies on lecithin membrane models (liposomes). Antimicrob. Ag. Chemother. 4:309-315.

Jarvis, B. 1983. Mould and mycotoxins in mouldy cheese. Microbiologie, Aliments, Nutrition. 1:187-191.

Jarzebski, A., Falkowski, L., and Borowski, E. 1982. Synthesis and structure-activity relationships of amides of amphotericin B. J. Antibiotics. 35:220-229.

Joint FAO/WHO Expert Committee on Food Additives. 1976. Evaluation of certain food additives. World Health Organization Technical Report Series, No. 599. 7-32.

Jukes, D. 12 November 1998. Personal Communication. The University of Reading, United Kingdom. 
Keim, G.R., Poutsiaka, J.W., Kirpan, J., Keysser, J.H. 1973. Amphotericin B methyl ester hydrochloride and amphotericin B: Comparative acute toxicity. Science. 179:584-585.

Keim, G.R., Sibley, P.L., Yoon, Y.H., Kulesza, J.S., Zaidi, I.H., Miller, M.M., and Poutsiaka, J.W. 1976. Comparative toxicological studies of amphotericin B methyl ester and amphotericin $\mathrm{B}$ in mice, rats, and dogs. Antimicrob. Ag. Chemother. 10:687-690.

Kinsky, S.C. 1967. Polyene antibiotics. Ch, In Antibiotics - Mechanisms of Action, Vol. 1 D. Gottlieb and P.D. Shaw (Ed.), p. 122-141. Springler-Verlag Inc. New York.

Klis, J.B., Witter, L.D., and Ordal, Z.J.. 1959. The effect of several antifungal antibiotics on the growth of common food spoilage fungi. Food Technol. 13:124-127.

Lawrence, R.M. and Hoeprich, P.D. 1976. Comparison of amphotericin B and amphotericin B methyl ester: Efficacy in murine coccidioidomycosis and toxicity. J. Infect. Dis. 133:168-174.

Lawrence, R.M., Hoeprich, P.D., Jagdis, F.A., Monji, N., Huston, A.C., and Schaffner, C.P. 1980. Distribution of doubly radiolabelled amphotericin B methyl ester and amphotericin B in the non-human primate, Macaca mulatta. J. Antimicrob. Chemother. 6:241-249.

Lechevalier, H., Borowski, E., Lampen, J.O. and Schaffner, C.P. 1961. Water-soluble $N$-acetyl derivatives of heptaene macrolide antifungal antibiotics: Microbiological studies. Antibiot. And Chemother. 11:640-647.

Levinskas, G.J., Ribelin, W.E., and Shaffer, C.B. 1966. Acute and chronic toxicity of pimaricin. Toxicol. Appl. Pharmacol. 8:97-109.

Lück, E. and Cheeseman, C.E. 1978. Mould growth on cheese as influenced by pimaricin or sorbate treatments. South African J. of Dairy Technol. 10:143-146. 
Lück, E. and Jager, M. 1997. Antimicrobial Food Additives, $2^{\text {nd }}$ ed. Springer-Verlag, New York, p. 214-218.

Lucy, J.A. and Glauer, A.M.. 1964. Structure and assembly of macromolecular lipid complexes composed of globular micelles. J. Mol. Biol. 8:727-748.

Lund, F., Filtenborg, O., and Frisvad, J.C. 1995. Associated mycoflora of cheese. Food Microbiol. 12:173-180.

MacPherson, D.T., Corbett, D.F., Costello, B.C., Driver, M.J., Greenlees, A.R., MacLachlan, W.S., Shanks, C.T., and Taylor, A.W. 1993. Adventures in polyene macrolide chemistry: The derivatisation of amphotericin B. Ch 14, In Recent Advances in the Chemistry of Anti-infective Agents. P.H. Bentley and R. Ponsford (Ed.), p. 205-222. The Royal Society of Chemistry, Cambridge, England.

Marquardt, R.R. and Frohlich, A.A. 1990. Ochratoxin A: an important western Canadian storage mycotoxin. Can. J. Phys. And Pharm. 68:991-999.

Marquardt, R.R. and Frohlich, A.A. 1992. A review of recent advances in understanding ochratoxicosis. J. Animal Sci. 70:3968-3988.

McMurry, J. 1992. Organic Chemistry, $3^{\text {rd }}$ ed., Brooks/Cole Publishing Co., Pacific Grove, California. 1041.

Mechlinski, W. and Schaffner, C.P. 1972. Polyene macrolide derivatives. I. N-acylation and esterification reactions with amphotericin B. J. Antibiotics. 25:256-258. 
Michalska-Trenkner, E. 1970. Induced resistance of Candida albicans strains to sodium salt of $N$-succinyl perimycin. Chemotherapy. 15:19-25.

Monji, N., Bonner, D.P., Hashimoto, Y., and Schaffner, C.P. 1975. Studies on the absorption, distribution, and excretion of radioactivity after intravenous and intraperitoneal administration of ${ }^{14} \mathrm{C}$-methyl ester of amphotericin B. J. Antibiot. 28:317-324.

Morris, H.A. and Hart, P.A. 1978. Pimaricin - What is it?. Cult. Dairy Prod. Journ. 13:22-24.

Norman, A.W., Demel, R.A., De Kruyff, B., and Van Deenen, L.L.M. 1972a. Studies on the biological properties of polyene antibiotics. J. Biol. Chem. 247:1918-1929.

Norman, A.W., Demel, R.A., De Kruyff, B., Geurts Van Kessel, W.S.M., and Van Deenen, L.L.M. 1972b. Studies on the biological properties of polyene antibiotics: Comparison of other polyenes with filipin in their ability to interact specifically with sterol. Biochim. Biophys. Acta. 290:1-14.

Northolt, M.D., Egmond, H.P. van, and Paulsch, W.E. 1979. Ochratoxin A production by some fungal species in relation to water activity and temperature. J. Food Prot. 42:485-490.

Northolt, M.D., Egmond, H.P. van, Soentoro, P., and Deijll, E. 1980. Fungal growth and the presence of sterigmatocystin in hard cheese. J. Assoc. Off. Anal. Chem. 63:115-119.

Oblack, D.L., Hewitt, W.L., and Martin, W.J. 1981. Comparative in vitro susceptibility of yeasts to amphotericin B and three methyl ester derivatives. Antimicrob. Ag. Chemother. 19:106-109.

Oostendorp, J.G. 1981. Natamycin. Antonie van Leeuwenhoek. 47:170-171. 
Pandey, R.C. and Rinehart, Jr., K.L. 1977. Polyene antibiotics. IX. An improved method for the preparation of methyl esters of polyene antibiotics. J. Antibiotics. 30:158-162.

Parmegiani, R.M., Loebenberg, D., Antonacci, B., Yarosh-Tomaine, T., Scupp, R., Wright, J.J., Chu, P.J.S., and Miller, G.H. 1987. Comparative in vitro and in vivo evaluation of $N$-D-ornithyl amphotericin B methyl ester, amphotericin B methyl ester, and amphotericin B. Antimicrob. Ag. Chemother. 31:1756-1760.

Perry, T. 6 November 1998. "Fruit juice fraud and deception". http://www.uvm.edu/ tmperry/.

Pierce, A.M., Pierce, H.D., Unrau, A.M., and Oehlschlager, A.C. 1978. Lipid composition and polyene antibiotic resistance of Candida albicans mutants. Can. J. Biochem. 56:135-142.

Pitt, J.I. 1987. Penicillium viridicatum, Penicillium verrucosum, and production of ochratoxin A. Appl. Environ. Microbiol. 53:266-269.

Pitt, J.I. and Hocking, A.D. 1997. Fungi and Food Spoilage, $2^{\text {nd }}$ Ed. Blackie Academic and Professional, New York.

Pohlmeier, M.M. and Bullerman, L.B. 1978. Ochratoxin production by a Penicillium species isolated from cheese. In: Abstracts of papers presented at the sixty-fifth annual meeting of IAMFES. J. Food Prot. 41:828-833.

Posthuma, J. and Berends, W. 1960. Triplet-triplet transfer as a mechanism of a photodynamic reaction. Biochim. Biophys. Acta. 41:538-541.

Posthuma, J. and Berends, W. 1961. Energy transfer in aqueous solutions. Biochim. Biophys. Acta. 51:390-392. 
Primary Care Optometry News. 26 February 1999. "Infection-causing organism dictates microbial keratitis treatment". http://www.slackinc.com/eye/pcon/199603/kera.htm.

Purdue Research Foundation. 23 April 1998. "Antifungal agents". http://www.vet.purdue.edu/depts/bms/courses/bms514/chmrx/antifung.htm.

Raab, W.P. 1972. Natamycin (Pimaricin) Its Properties and Possibilities in Medicine. Georg Thieme Publishers Stuttgart, London.

Schaffner, C.P. and Borowski, E. 1961. Biologically active $N$-acyl derivative of polyene macrolide antifungal antibiotics. Antibiot. And Chemother. 11:724-732

Schaffner, C.P. and Mechlinski, W. 1972. Polyene macrolide derivatives. II. Physical-chemical properties of polyene macrolide esters and their water soluble salts. J. Antibiotics. 25:259-260.

Schaffner, C.P. 1987. Amphotericin derivatives. Ch, In Recent Trends in the Discovery, Development and Evaluation of Antifungal Agents, Proceedings of an International Telesymposium, May 1987. R.A. Fromtling, (Chair), 595-618. J.R. Prous Science Publishers, Barcelona, Spain.

Schaffner, C.P. 19 May 1998. Personal Communication. Waksman Institute of Microbiology, Rutgers - The State University of New Jersey, Piscataway, New Jersey.

Shahani, K.M., Bullerman, L.B., Barnhart, H.M., and Hartung, T.E. 1973. Effect of an antifungal, pimaricin, upon the retardation of food spoilage and incidence of toxins. Abstracts in Int. Congress for Bacteriology. 41.

Sinha, A.K. and Ranjan, K.S. 1991. A report of mycotoxin contamination in Bhutanese cheese. J. Food Sci. and Technol. 28:398-399. 
Sipos, F. and Keseleski, A.J. 1977. Amphotericin B methyl ester salts. U.S. Patent 4,041,232.

Sokol-Anderson, M., Sligh, J.E., Elberg, S., Brajtburg, J., Kobayashi, G.S., and Medoff, G. 1988. Role of cell defense against oxidative damage in the resistance of Candida albicans to the killing effect of amphotericin B. Antimicrob. Ag. Chemother. 32:702-705.

Stefańska, B., Bylec, E., and Borowski, E. 1980. The synthesis of amides of polyene macrolide antibiotics. J. Antibiotics. 33:103-104.

Stock, R. 1981. How effective are antimycotic drugs?. Phar. Int. October 1981:232-236.

Struyk, A.P. and Waisvisz, J.M. 1960. Pimaricin and process of producing the same. British Patent 844,289.

Struyk, A.P. and Waisvisz, J.M. 1975. Pimaricin and process of producing the same. U.S. Patent $3,892,850$.

Struyk, A.P., Hoette, I., Drost, G., Waisvisz, J.M, Eek, T. van, and Hoogerheide, J.C. 1958. Pimaricin, a new antifungal antibiotic. Antibiot. Ann. 878-885.

Subden, R.E., Safe, L., Morris, D.C., Brown, R.G., and Safe, S. 1977. Eburicaol, lichesterol, ergosterol, and obtusifoliol from polyene antibiotic-resistant mutants of Candida albicans. Can. J. Microbiol. 23:751-754.

Thomas, A.H. 1976. Analysis and assay of polyene antifungal antibiotics. The Analyst. 101:321339.

Vandeputte, J. 1973. Borate complex of polyene macrolide antibiotics. U.S. Patent 3,740,424. 
Webber, M.M. and Kinsky, S.C. 1965. Effect of cholesterol on the sensitivity of Mycoplasma laidlawii to the polyene antibiotic filipin. J. Bacteriol. 89:306-312.

Weissmann, G. and Sessa, G. 1967. The action of polyene antibiotics on phospholipidcholesterol structures. J. Biol. Chem. 242:616-625.

Woods, R.A. 1971. Nystatin resistant mutants of yeast: Alterations in sterol content. J. Bact. 108:69-73.

Ziogas, B.N., Sisler, H.D., and Lusby, W.R. 1983. Sterol content and other characteristics of pimaricin-resistant mutants of Aspergillus nidulans. Pestic. Biochem. Physiol. 20:320-329.

Zutphen, H.V., Demel, R.A., Norman, A.W., and Van Deenen, L.L.M. 1971. The action of polyene antibiotics on lipid bilayer membranes in the presence of several cations and anions. Biochim. Biophys. Acta. 241:310-330.

Zygmunt, W.A. 1966. Intracellular loss of potassium in Candida albicans after exposure to polyene antifungal antibiotics. Appl. Microbiol. 14:953-956. 


\title{
CHAPTER 2: SYNTHESIS OF $N$-AMINOACYL DERIVATIVE OF NATAMYCIN
}

\begin{abstract}
The polyene macrolide antibiotic natamycin (Antibiotic A-5283) is commonly used to prevent the growth of surface molds on different cheese varieties. Natamycin is commonly applied to the surface of cheese by dipping or spraying, using an aqueous solution containing 200 to $300 \mathrm{ppm}$ of the additive (CFR, 1998a). The large molecular weight of natamycin, 666 $\mathrm{g} / \mathrm{mol}$, and conjugated double bond structure causes it to be extremely insoluble in water and most food grade solvents. The inability to apply natamycin in true solution creates void nontreated areas on the food surface. These non-treated areas promote the growth of fungal organisms. Altering the chemical structure of natamycin to make it more water soluble could increase its effectiveness in retarding the growth of surface mold. A water soluble $N$-alkyl semisynthetic derivative of natamycin was synthesized by the Michael addition reaction of the parent with an $\mathrm{N}$-substituted malemide. The use of a water soluble natamycin semisynthetic derivative as a preservation agent may drastically increase the shelf-life of shredded cheese.
\end{abstract}

\section{Introduction}

The effectiveness of natamycin to prevent or retard surface mold growth is limited by its ability to be evenly distributed on the food surface and its stability in the food system. In its native form, natamycin exists as an extremely insoluble crystal (Oostendorp, 1981). Each crystal imparts a zone of inhibition which successfully prevents or retards the growth of mycotic organisms. In an ideal situation, natamycin crystals will be distributed on a food surface in a manner in which all the zones of inhibition overlap. Unfortunately, this ideal situation can not be achieved because of natamycin's physical properties.

The insolubility of natamycin is particularly problematic for cheese producers because of the manner in which natamycin is applied to the surface of cheese. For instance, a 200 to 300 ppm solution of natamycin is typically sprayed onto shredded cheese during tumbling (CFR, 
1998). Because of natamycin's relative insolubility in water, less than 5 ppm of natamycin actually enters into true solution and is applied in a proficient manner (Schaffner and Mechlinski, 1972).

Natamycin's solubility can be improved by chemical modification. Medical research shows that chemical modification of the free carboxyl and amino functional groups of polyene macrolide antibiotics have produced compounds with increased selective toxicity and solubility in water. Czerwiński et al. (1991) prepared an $N$-alkyl derivative of amphotericin B (AmB) by reacting the parent antibiotic with an $N$-substituted malemide. Synthesis of the semisynthetic derivative followed the Michael addition reaction. Minimum inhibitory concentration (MIC) studies for this new antibiotic showed that it was more active against Aspergillus niger, Penicillium cytrinum, and Mucor mucedo. Since $82 \%$ of molds on refrigerated cheese belongs to the genus Penicillium and $7 \%$ to the genus Aspergillus a similar semisynthetic derivative may extend the shelf-life of cheeses (Bullerman, 1981).

In addition to the selective nature of the AmB derivative, it is believed that resistance and tolerance of fungal organisms to this or any other polyene macrolide would be of no clinical importance. After nearly 40 years of clinical use, reports of fungi resistant to AmB are infrequent (Brajtburg et al., 1990). In one experiment, strains of molds isolated in cheese warehouses showed no decrease in sensitivity to natamycin after numerous transfers of the culture was subjected to sub-lethal doses of the antibiotic. No differences were found in natamycin sensitivity between fungal populations for warehouses utilizing natamycin for 10 years and warehouses that never used the antimycotic (De Boer and Stolk-Horsthuis, 1977).

It is also hypothesized that the acute and chronic toxicity of water soluble polyene macrolide antibiotics is lower than that of their parents (Lawrence and Hoeprich, 1976). Lower toxicity would be expected for water soluble compounds because their increased water solubility would prevent their accumulation in various tissues, e.g., the kidneys (Schaffner, 1987).

The uniqueness of the AmB derivative is the presence of an additional, basic nitrogen atom in the modified aminosugar moiety (Czerwiński et al., 1991). The nitrogen atom in this derivative is protonable to ensure the formation of a hydrogen bond with the oxygen atom of a sterol hydroxyl group. The destructive mode of action of polyene macrolides is their ability to 
form antibiotic-sterol complexes (Hamilton-Miller, 1974). These compounds disrupt cell membrane permeability causing the loss of inorganic ions and metabolites. Loss of these constituents cause death to the cell.

Chéron et al. (1988) conducted a quantitative structure-activity relationship study for polyene macrolide antibiotics which revealed information on polyene macrolide antibiotics sterol specificity. Results from the study showed that the lack of free carboxyl group in the molecules favors the differentiation between cholesterol and ergosterol containing cells. The procedure Czerwiński et al. (1991) used to prepare their AmB derivative ensured the protonation of the free carboxyl group allowing for increased sterol specificity of ergosterol. The formation of antibiotic-ergosterol complexes are much more destructive to cells than other, particularly cholesterol, complexes.

The objective of this research was to synthesize a semisynthetic derivative with increased water solubility and stability. Presently, the dairy industry has difficulty predicting the shelf-life of high surface area processed cheese. Berry (1999) states that the greatest obstacle shredded cheese manufacturers face is product return due to mold growth. Natamycin's ability to retard mold growth on processed cheese is unpredictable due to its limited solubility. The effectiveness of natamycin to prevent mold growth on cheese would be more predictable if its application was more uniform. A more soluble natamycin derivative would allow better application to the surface of cheese and may retard mold growth in a more efficient manner.

\section{Materials and Methods}

\section{Synthesis of Propylaminomalemide (PAM) Hydrochloride}

$N$-(3-dimethylaminopropyl)maleimide hydrochloride was synthesized according to known procedure (Brantley, 1984). For this experiment, 3-dimethylaminopropylamine (10.2 g, $100 \mathrm{mmol}$ ) was placed in a $100 \mathrm{ml}$ flask with mechanical stirring. Maleic anhydride $(9.8 \mathrm{~g}, 100$ mmol) was added in portions and the temperature was maintained at $0-5^{\circ} \mathrm{C}$ by the addition of ice. When most of the anhydride was dissolved, the mixture was allowed to warm until solution 
was complete. Water was removed with a rotary film evaporator (Schott Duran Evapotec ${ }^{\mathrm{TM}}$, West Germany). Acetic anhydride $(16 \mathrm{ml})$ was slowly added to the viscous syrup. Flask was placed in an ice bath to maintain temperature of solution below $60^{\circ} \mathrm{C}$. Solution was allowed to stand for 12 hours.

Ethanol $(25 \mathrm{ml})$ was added and the mixture was evaporated with a rotary film evaporator to remove excess acetic anhydride. The residue was dissolved in anhydrous ethanol (25 $\mathrm{ml})$ and gaseous hydrogen chloride was passed into the mixture until it was strongly acidic $(\mathrm{pH}<3)$. Excess anhydrous ethyl ether was added to the solution to precipitate the product. Ether was removed and anhydrous ethanol $(250 \mathrm{ml})$ was added. Solution was warmed to $50^{\circ} \mathrm{C}$ to fully dissolve precipitate in ethanol. Mixture was cooled to room temperature, undisturbed, and allowed to precipitate. Solution was filtered through porous filter paper (Whatman ${ }^{\mathrm{TM}}$ Filter Paper \#41, Whatman, Inc., Clifton, NJ) in a Buchner funnel and collected in an amber sample jar. Product was dried in a vacuum oven (Fisher Isotemp® Vacuum Oven Model 281, Fisher Scientific, Pittsburgh, PA) at $35^{\circ} \mathrm{C}$ overnight.

\section{Synthesis of Natamycin Derivative}

Synthesis was modeled after a similar procedure for AmB (Czerwiński et al., 1991). Natamycin was obtained in a 2.5\% sterile water suspension (Aldrich, Milwaukee, WI). Suspension was freeze dried at $25^{\circ} \mathrm{C}$ using a laboratory freeze dryer (Virtis, Gardiner, NY). Synthesis of natamycin derivative proceeded by the Michael addition reaction. Natamycin (4 g, $6 \mathrm{mmol})$ was dissolved in dimethylformamide $(100 \mathrm{ml})$ and triethylamine $(1 \mathrm{ml}, 0.14 \mathrm{mmol})$.

PAM hydrochloride $(6.56 \mathrm{~g}, 36 \mathrm{mmol})$ and a few drops of dimethylaminopyridine $(0.1 \mathrm{ml})$ were added slowly to the mixture. The mixture was stirred and subjected to a constant overpressure of argon for 12 hours. Reaction was protected from light.

Anhydrous ethyl ether $(250 \mathrm{ml})$ was added to the mixture to precipitate the product. Ether was removed and product was dried in vacuum oven at $35^{\circ} \mathrm{C}$ overnight. Crude product was then purified by column chromatography ( silica $\left(\mathrm{SiO}_{2}\right)$; chloroform $\left(\mathrm{CHCl}_{3}\right)$ - methanol $(\mathrm{MeOH}), 13: 7)$. Thin layer chromatography was performed to monitor purification. Solvent 
was removed from product in a rotary film evaporator and dried in a vacuum oven at $35^{\circ} \mathrm{C}$ overnight.

\section{Results and Discussion}

The structure confirmation of natamycin and PAM hydrochloride was obtained before synthesis of derivative (Figures 1 and 2). The low resolution fast atom bombardment (FAB) mass spectra of natamycin demonstrated a well represented parent ion. Major ions represented in spectra included $666(\mathrm{M}+\mathrm{H})^{+}, 648\left(\mathrm{M}+\mathrm{H}_{-} \mathrm{H}_{2} \mathrm{O}\right)^{+}, 504(\mathrm{M}+\mathrm{H} \text {-aminosugar })^{+}$. The parent ion of PAM hydrochloride, $182(\mathrm{M}+\mathrm{H})^{+}$, was seen in the low resolution FAB mass spectra of PAM hydrochloride. NMR analysis confirmed the mass spectra results for PAM hydrochloride. The ${ }^{1} \mathrm{H}-\mathrm{NMR}$ spectra of PAM hydrochloride showed, in addition to other signals, $\delta=2.72 \mathrm{ppm}(\mathrm{s})$ $(6 \mathrm{H})$, representing the $\mathrm{N}$-methyl groups, and $\delta=6.72 \mathrm{ppm}(\mathrm{s})(2 \mathrm{H})$ representing the vinyl protons of the malemide ring. The structure confirmation of the natamycin derivative, $N$ (3'-N-dimethylaminopropylsuccimido) natamycin, was confirmed by high and low resolution mass spectroscopy and NMR analysis (Figures 1 and 2). Major ions represented in the low resolution FAB mass spectra for the derivative included $848(\mathrm{M}+\mathrm{H})^{+}, 870(\mathrm{M}+\mathrm{Na})^{+}$, and 686 $(\mathrm{M}+\mathrm{H} \text {-aminosugar })^{+}$. The high resolution mass spectra showed the presence of the parent ion, $848(\mathrm{M}+\mathrm{H})^{+}$. The ${ }^{1} \mathrm{H}-\mathrm{NMR}$ spectra of $N$-alkyl natamycin showed the signal, $\delta=2.35 \mathrm{ppm}(\mathrm{s})$ $(6 \mathrm{H})$, representing the $\mathrm{N}$-methyl groups, but did not show any signals indicating vinyl protons of the malemide ring. These results suggest that no free PAM hydrochloride was present and the proposed $N$-alkyl natamycin compound was intact.

\section{Conclusions}

Previous researchers synthesized active water soluble AmB derivatives inclusive with a protonable nitrogen atom by the Michael addition reaction (Czerwiński et al., 1991). This route can also be used to synthesize natamycin derivatives with several modifications. The addition of dimethylaminopyridine allowed reaction to proceed more quickly and acted as a catalyst. 
Purification of crude product was enhanced by using column chromatography $\left(\mathrm{SiO}_{2} ; \mathrm{CHCl}_{3}\right.$ $\mathrm{MeOH}, 13: 7)$ and showed better results then counter-current system.

Natamycin derivatives may have widespread use in the food industry as preservative agents against surface mold growth. Currently, natamycin is the only antibiotic approved for the direct addition to food for human consumption by the FDA. The selective nature, low relative toxicity, and ability to remain active against potential resistant organisms makes natamycin an ideal food preservative. The limiting factors for natamycin is its ability to be evenly distributed on a food surface and stability in the food system. These limiting factors can be overcome by chemical modification. Additional research must be performed to better understand how these chemical modifications will effect the efficacy, selectivity, stability, and toxicity of these antibiotic derivatives.

In addition, processing parameters must be analyzed and optimized for natamycin and natamycin derivatives. The development of tailor made solvent systems for specific antibiotics warrants investigation. Solvent systems incorporated with food grade additives that preserve the stability of natamycin and promotes further solubility is another approach to improve the effectiveness of natamycin and like preservatives. Also, molecular studies for natamycin and semisynthetic derivatives are needed to better understand the antimycotic action taken place at the antibiotic-food interface. 
Tables

Table 1: Major ions present in the positive ion fast atom bombardment mass spectras for natamycin, propylaminomalemide hydrochloride, and natamycin derivative

\begin{tabular}{|c|c|c|c|}
\hline Compound & Formula & MW & Major Ions (m/z) \\
\hline Natamycin & $\mathrm{C}_{33} \mathrm{H}_{47} \mathrm{O}_{13} \mathrm{~N}$ & 665 & $666(\mathrm{M}+\mathrm{H})^{+}, 648\left(\mathrm{M}+\mathrm{H}_{-} \mathrm{H}_{2} \mathrm{O}\right)^{+}$, \\
& & & $504(\mathrm{M}+\mathrm{H} \text {-aminosugar })^{+}$ \\
\hline PAM & $\mathrm{C}_{9} \mathrm{H}_{15} \mathrm{O}_{2} \mathrm{~N}_{2}$ & 184 & $182(\mathrm{M}-2 \mathrm{H})^{+}$ \\
Hydrochloride & & & $848(\mathrm{M}+\mathrm{H})^{+}, 870(\mathrm{M}+\mathrm{Na})^{+}$, \\
\hline $\begin{array}{c}\text { Natamycin } \\
\text { Derivative }\end{array}$ & $\mathrm{C}_{42} \mathrm{H}_{61} \mathrm{O}_{15} \mathrm{~N}_{3}$ & 847 & $686(\mathrm{M}+\mathrm{H} \text {-aminosugar })^{+}$ \\
\hline
\end{tabular}

Table 2: ${ }^{1}$ H NMR spectral data for propylaminomalemide hydrochloride and natamycin derivative

\begin{tabular}{|c|c|c|c|}
\hline Compound & Formula & $\delta(\mathrm{ppm})$ & Affiliation \\
\hline PAM & $\mathrm{C}_{9} \mathrm{H}_{15} \mathrm{O}_{2} \mathrm{~N}_{2}$ & 2.72 & $(6 \mathrm{H})$ N-methyl groups \\
\cline { 3 - 4 } & & 6.72 & $(2 \mathrm{H})$ vinyl protons of the malemide ring \\
\hline Hydrochloride & & 2.35 & $(6 \mathrm{H})$ N-methyl groups \\
Derivamycin & $\mathrm{C}_{42} \mathrm{H}_{61} \mathrm{O}_{15} \mathrm{~N}_{3}$ & & \\
\hline
\end{tabular}




\section{References}

Berry, D. 1999. Natamycin for shredded cheese. Dairy Foods. 100:45.

Brajtburg, J., Powderly, W.G., Kobayashi, G.S., and Medoff, G. 1990. Amphotericin B: Current Understanding of Mechanisms of Action. Antimicrob. Ag. Chemother. 34:183-188.

Brantley, R.K., Haeffner-Gormley, L., and Wetlaufer, D.B. 1984. Preparation of a positively charged malemide and its application to the high-performance liquid chromatographic separation of the tryptic peptides of lysozyme. J. Chromatogr. 295:220-225.

Bullerman, L.B. 1981. Public health significance of molds and mycotoxins in fermented dairy products. J. Dairy Sci. 64:2439-2452.

CFR, 1998. Code of Federal Regulations. Title 21. Part 172. U.S. Government Printing Office, Washington, DC.

Chéron, M., Cybulska, B., Mazerski, J., Grzybowska, J., Czerwiński, A., and Borowski, E. 1988. Quantitative structure-activity relationships in amphotericin B derivatives. Biochem. Pharmac. 37:827-836.

Czerwiński, A., König, A., Zieniawa, T., Sowiński, P., Sinnwell, V., Milewski, S., and Borowski, E. 1991. New $N$-alkyl derivatives of amphotericin B synthesis and biological properties. J. Antibiotics. 44:979-984.

De Boer, E. and Stolk-Horsthuis, M. 1977. Sensitivity to natamycin (pimaricin) of fungi isolated in cheese warehouses. J. Food Prot. 40:533-536. 
FR 1998. Federal Register. Vol. 63. p. 66,014. U.S. Government Printing Office, Washington, DC.

Hamilton-Miller, J.M.T. 1974. Fungal sterols and the mode of action of the polyene antibiotics. Adv. Appl. Microbiol. 17:109-134.

Lawrence, R.M. and Hoeprich, P.D. 1976. Comparison of amphotericin B and amphotericin B methyl ester: Efficacy in murine coccidioidomycosis and toxicity. J. Infect. Dis. 133:168-174.

Oostendorp, J.G. 1981. Natamycin. Antonie van Leeuwenhoek. 47:170-171.

Schaffner, C.P. and Mechlinski, W. 1972. Polyene macrolide derivatives. II. Physical-chemical properties of polyene macrolide esters and their water soluble salts. J. Antibiotics. 25:259-260.

Schaffner, C.P. 1987. Amphotericin derivatives. Ch., In Recent Trends in the Discovery, Development and Evaluation of Antifungal Agents, Proceedings of an International Telesymposium, May 1987. Fromtling, R.A. (Chair), 595-618. J.R. Prous Science Publishers, Barcelona, Spain. 


\section{Appendix}

Figure 1: Reaction scheme for the preparation of propylaminomalemide (PAM) hydrochloride (Brantley, 1984).
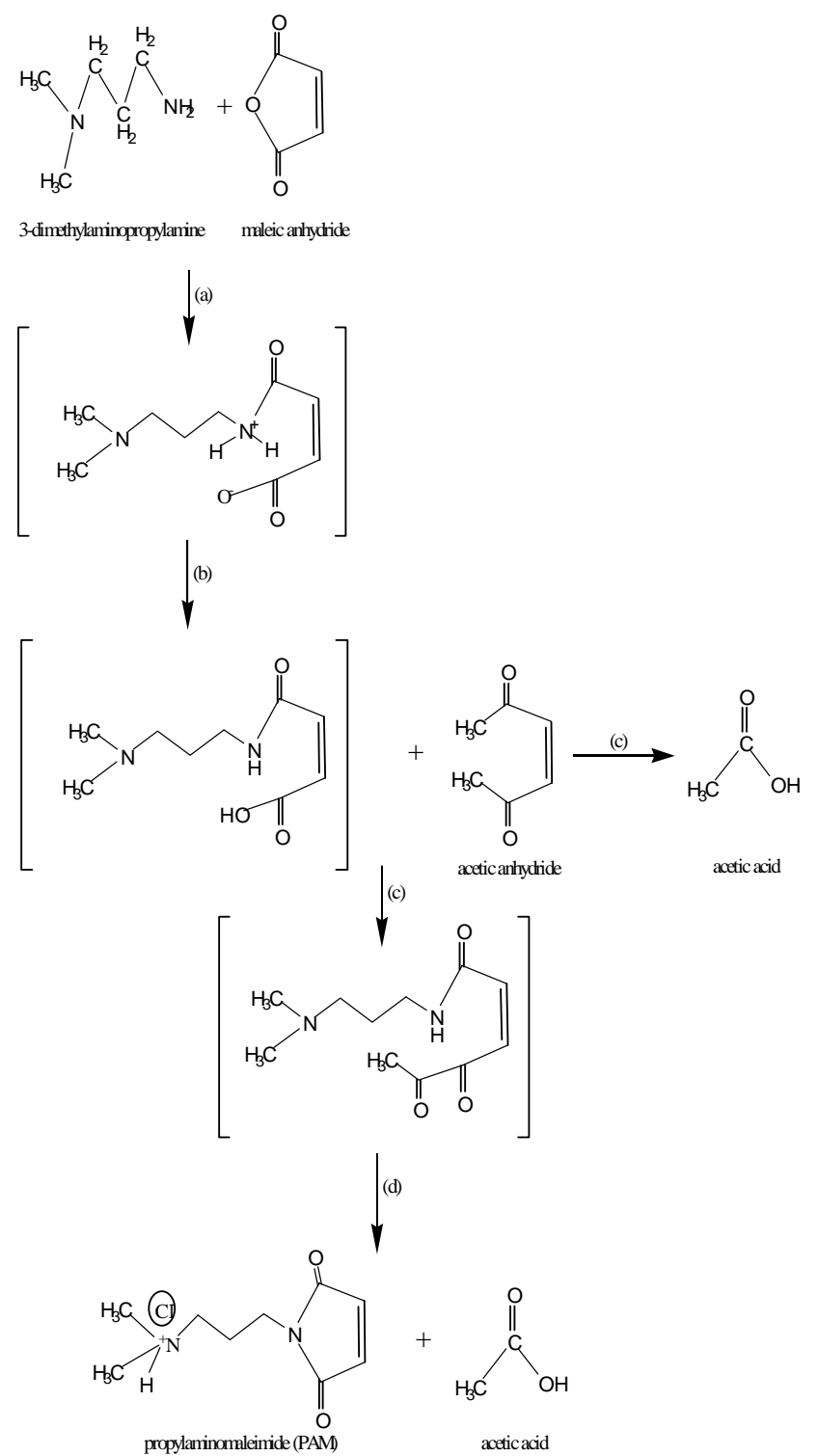

Scheme 1 (a) solid $\mathrm{H}_{2} \mathrm{O}$ (b) $\mathrm{H}_{2} \mathrm{O}$ removed in vacuo (c) i. $\mathrm{CH}_{3} \mathrm{CH}_{2} \mathrm{OH}$; ii. $\mathrm{CH}_{3} \mathrm{CH}_{2} \mathrm{OH}$ removed in vacuo (d) i. anhydrous $\mathrm{CH}_{3} \mathrm{CH}_{2} \mathrm{OH}$; ii. gaseous $\mathrm{HCl}$ iii. anhydrous $\left(\mathrm{C}_{2} \mathrm{H}_{5}\right)_{2} \mathrm{O}$. 
Figure 2: Reaction scheme for the preparation of $N-(3$ '-N-dimethylaminopropylsuccimido) natamycin (natamycin derivative)<smiles>CC(C/C=C/C=C/C=C/C=C/C(CC1OC(O)(CCO)CC(O)C1C(=O)O)OC1O[C@H](C)C(O)[C@@H](N)[C@H]1O)OC(=O)/C=C/C1OC1CO</smiles>

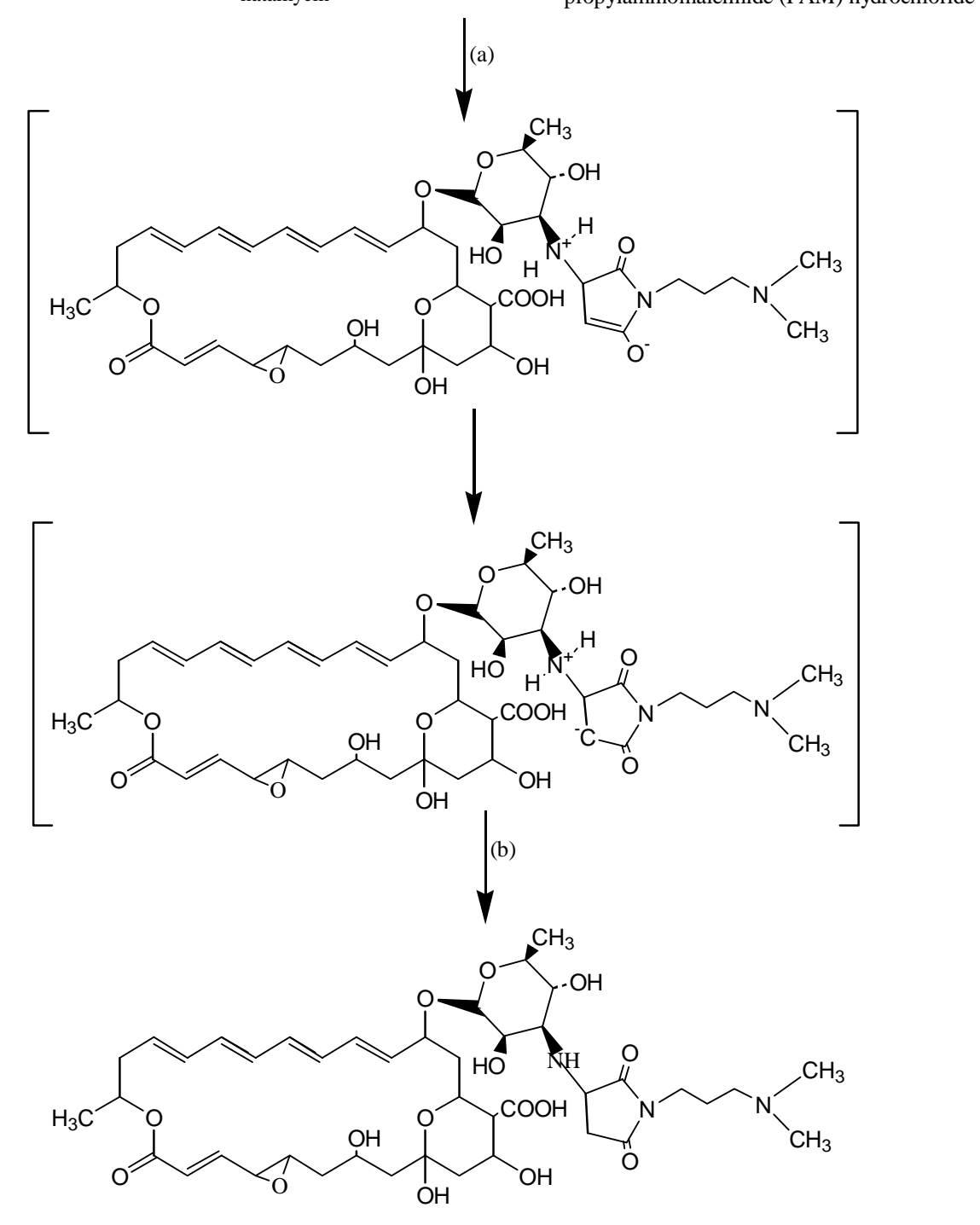

$\mathrm{N}$-(3'-N-dimethylaminopropylsuccimido) natamycin

Scheme 2 (a) i. DMF ii. gaseous $\mathrm{Ar}(\mathrm{b})$ anhydrous $\left(\mathrm{C}_{2} \mathrm{H}_{5}\right)_{2} \mathrm{O}$. 
Figure 3: Low resolution $F A B$ mass spectra of natamycin

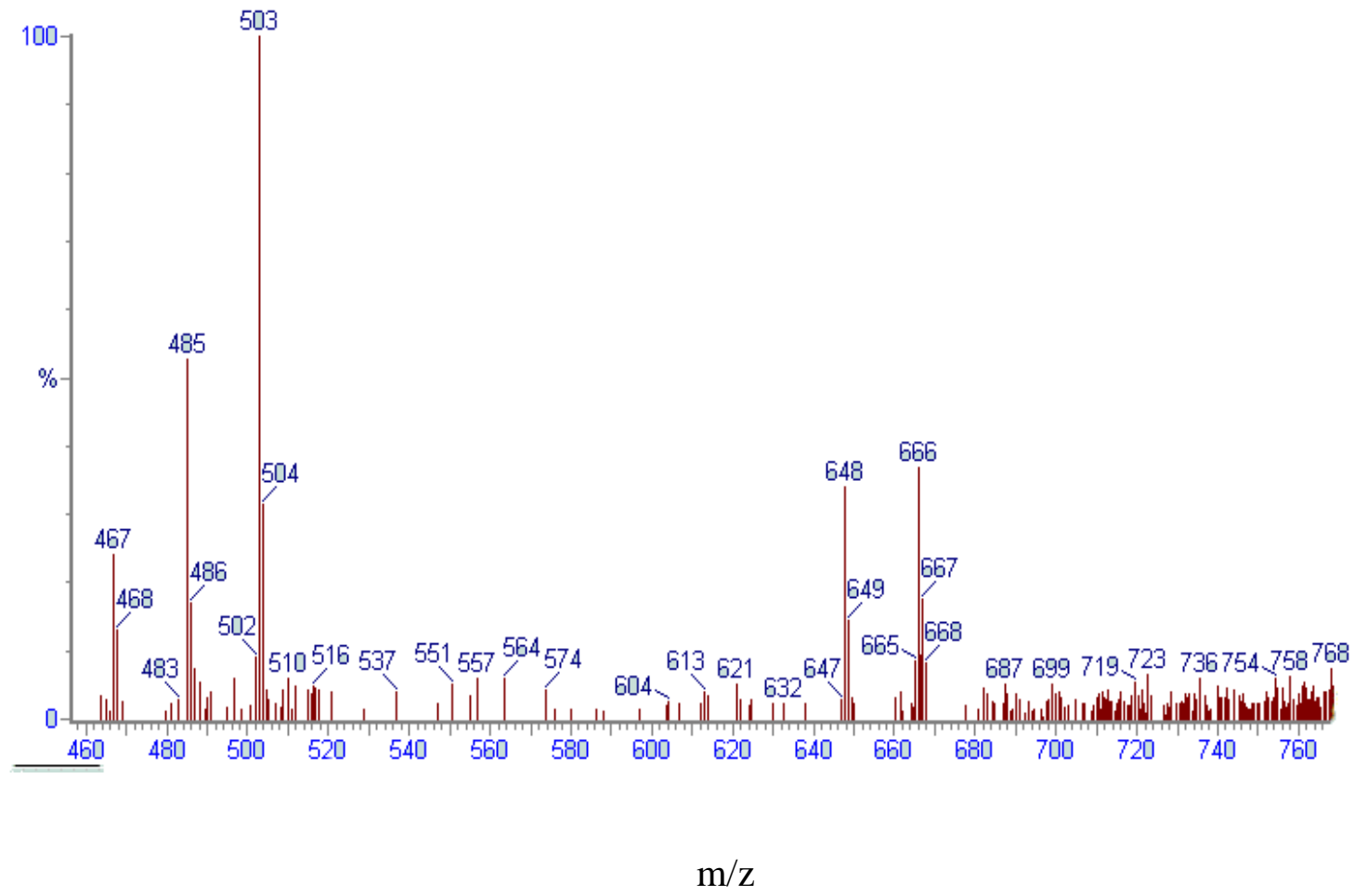


Figure 4: Low resolution FAB mass spectra of PAM hydrochloride

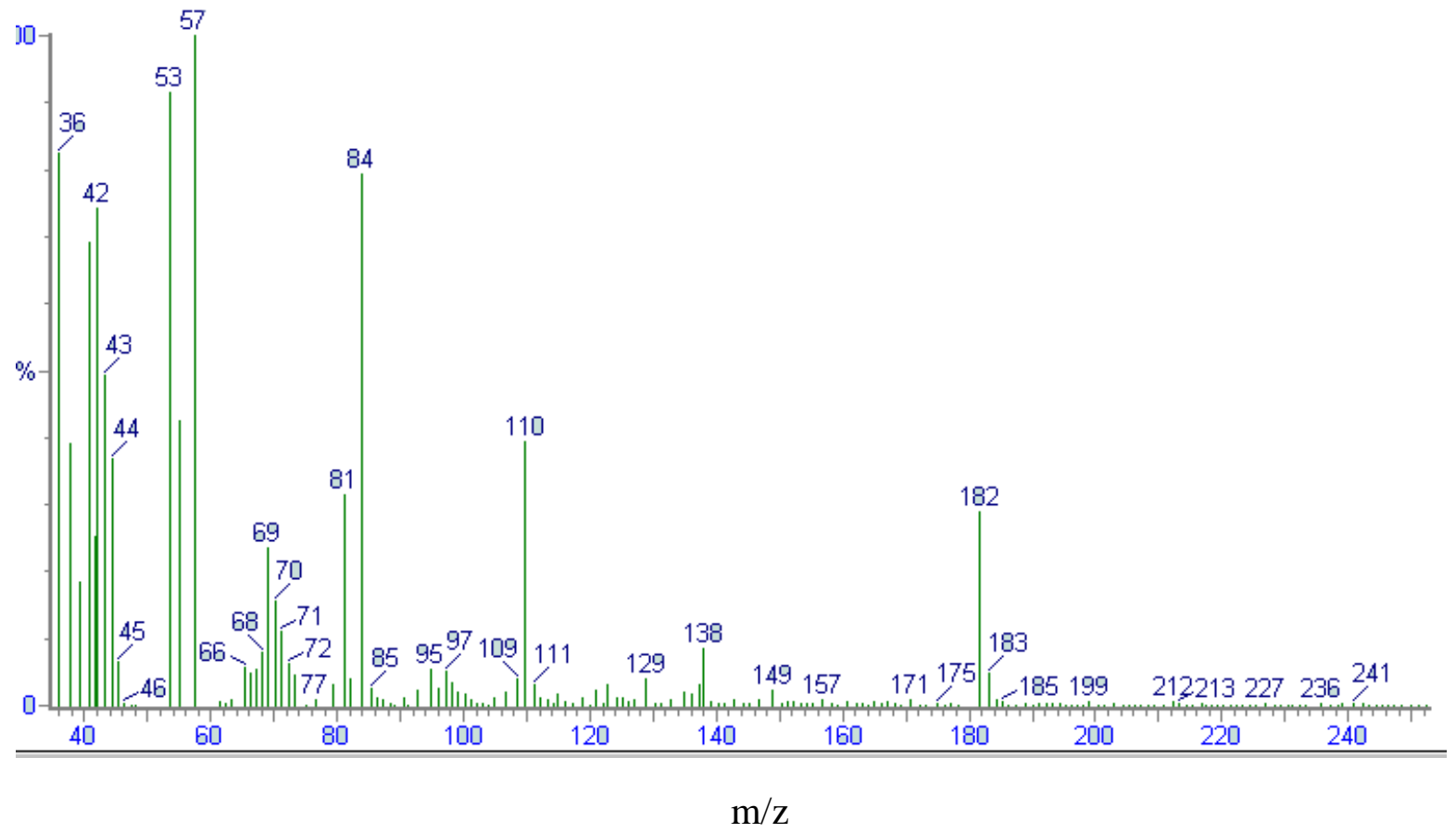


Figure 5: ${ }^{1} \mathrm{H}$-NMR spectra of PAM hydrochloride in $\mathrm{D}_{2} \mathrm{O}$

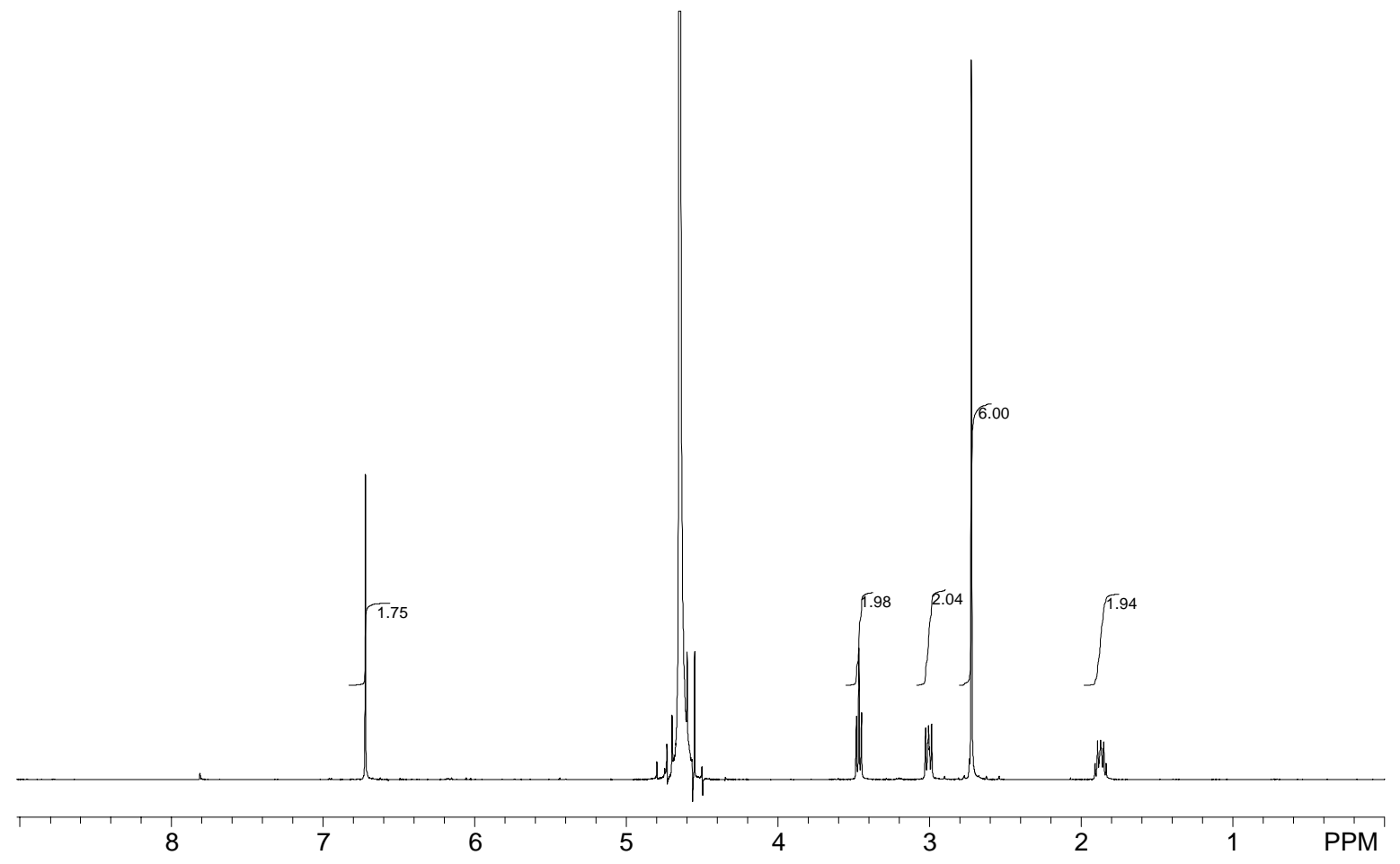


Figure 6: Low resolution FAB mass spectra of $N$-(3'-N-dimethylaminopropylsuccimido) natamycin (natamycin derivative)

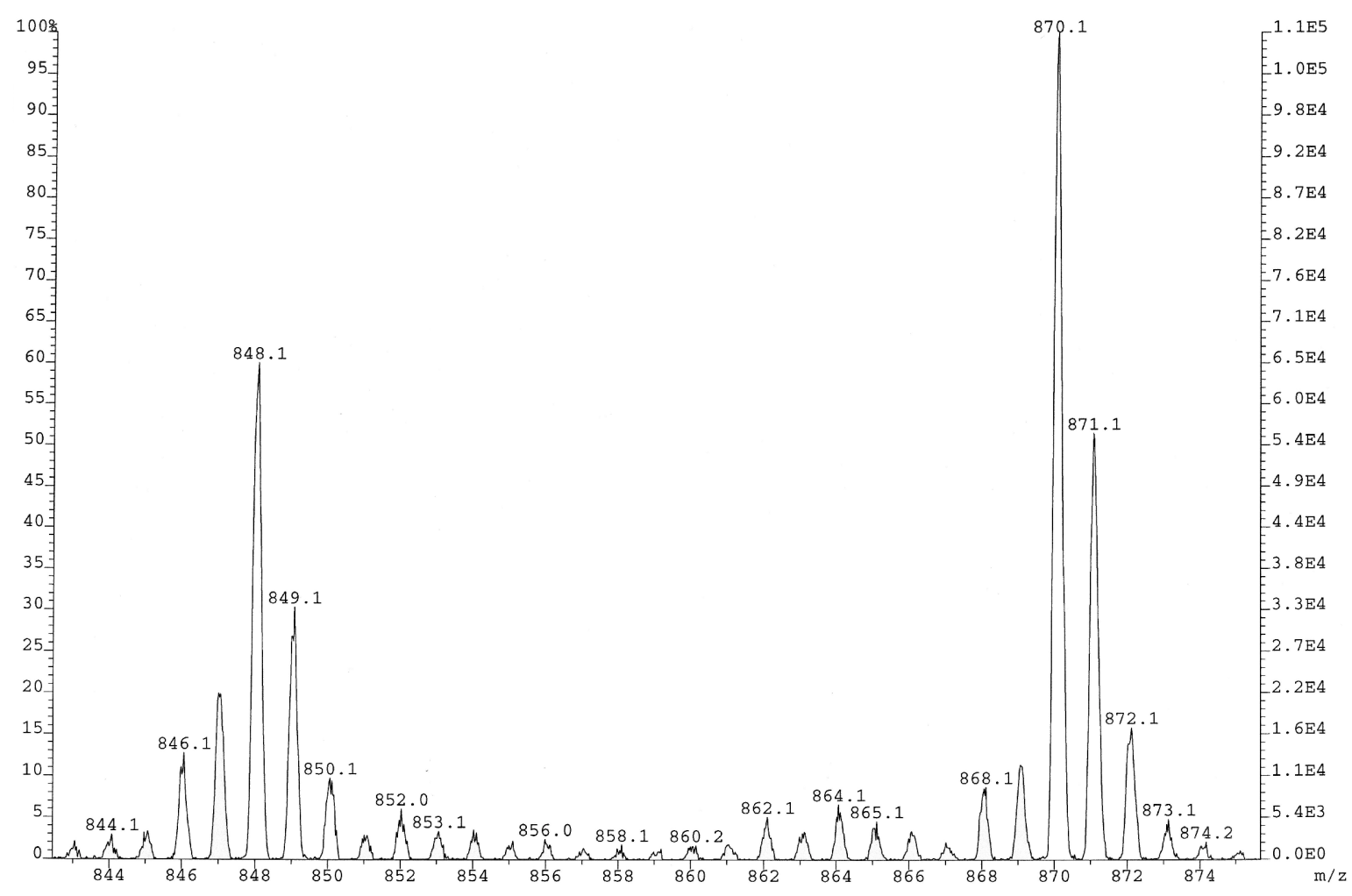


Figure 7: High resolution FAB mass spectra of $N$-(3'-N-dimethylaminopropylsuccimido) natamycin (natamycin derivative)

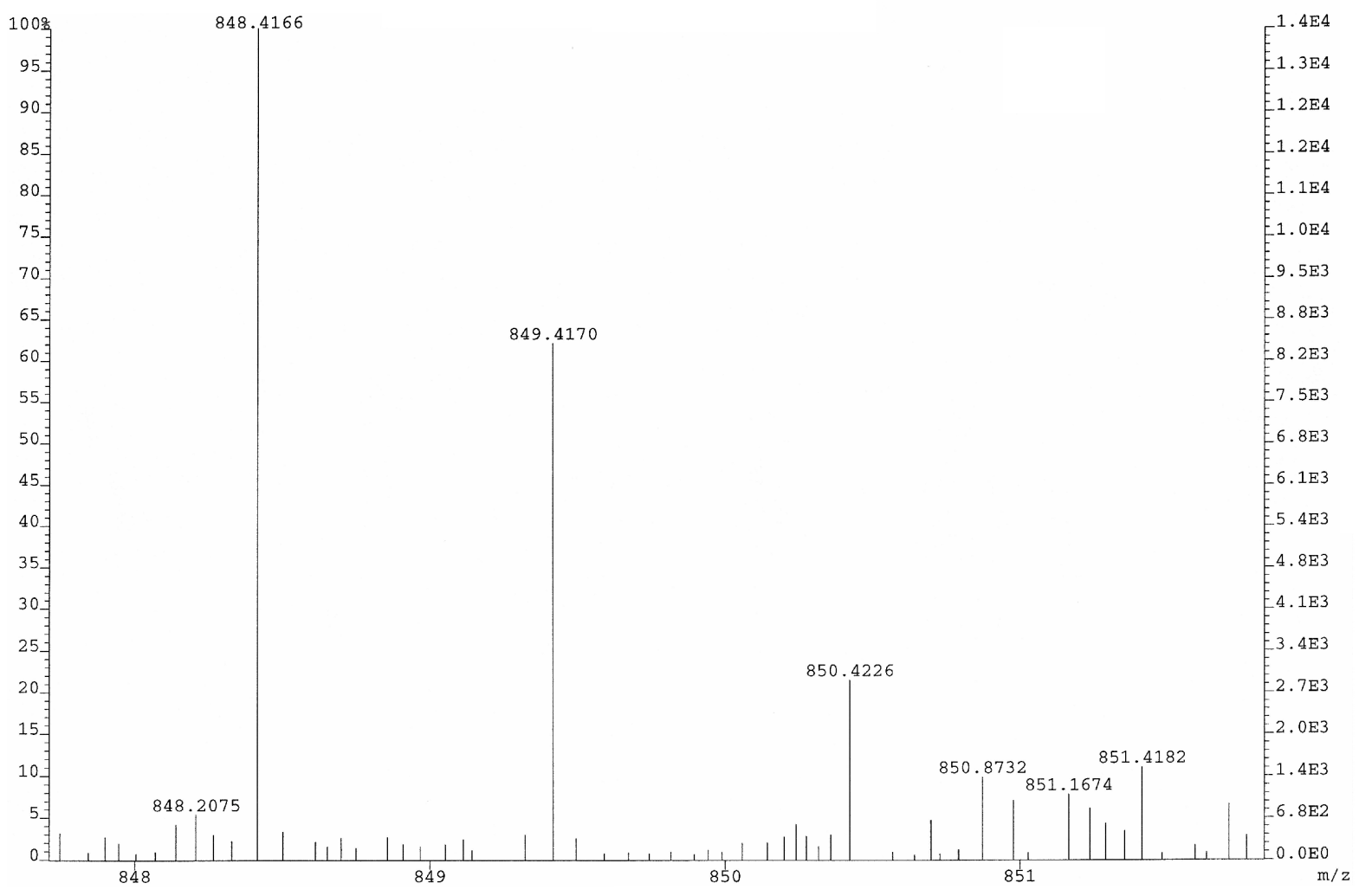


Figure 8: ${ }^{1} \mathrm{H}$-NMR spectra of $\mathrm{N}$-(3'-N-dimethylaminopropylsuccimido) natamycin (natamycin derivative) in pyridine- $d_{5}$

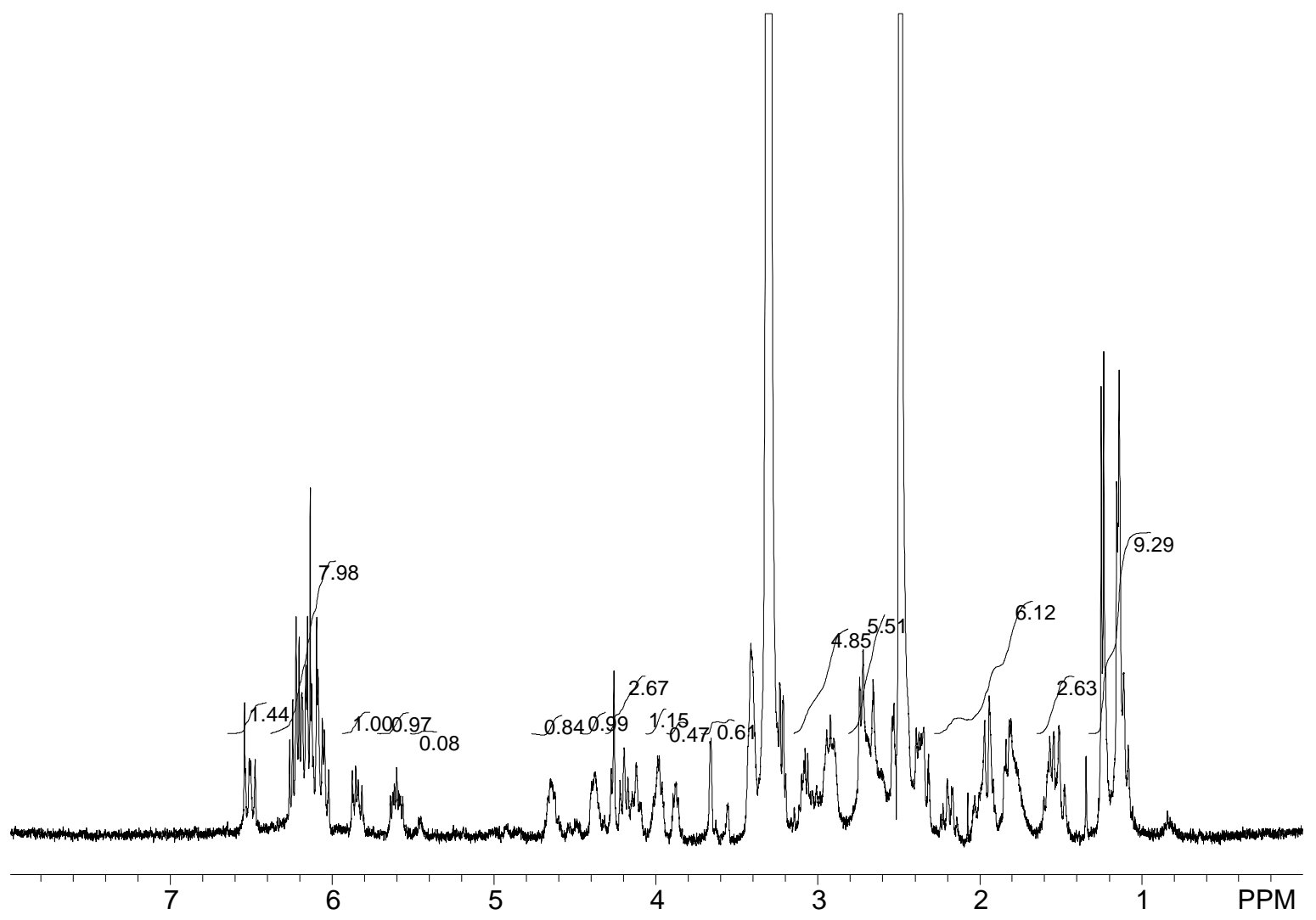




\title{
CHAPTER 3: MICROBIOLOGICAL CHALLENGE STUDY FOR SEMISYNTHETIC DERIVATIVE OF NATAMYCIN AND PARENT ANTIBIOTIC
}

\begin{abstract}
A comparative study investigating the effectiveness of a semisynthetic derivative of natamycin and the parent antibiotic in suppressing mold growth on one month aged shredded Cheddar cheese modified atmosphere packaged (MAP) was performed. A $20 \mathrm{ppm}$ natamycin treatment effectively suppressed visible mold growth $\left(<10^{4} \mathrm{CFU} / \mathrm{g}\right)$ for 30 days after opening in MAP samples. The $20 \mathrm{ppm}$ semisynthetic derivative performed similarly to the $10 \mathrm{ppm}$ natamycin treatment in retarding mold growth. Visible mold growth did not occur for these treatments until 20 days after opening MAP samples. Analysis of storage conditions revealed that an outgrowth of mold in shredded cheese occurs when MAP packages are stored longer than 15 days prior to opening. This bloom in mold growth is attributed to the degradation of natamycin and the semisynthetic derivative throughout storage.
\end{abstract}

\section{Introduction}

Natamycin is the only approved antimycotic agent that can be used to prevent the growth of mold on cheese. The use of sorbates will effectively retard the growth of mold on cheese, but common cheese spoilage organisms such as Aspergillus and Penicillium degrade sorbate through decarboxylation (Sofos and Busta, 1981; Marth et al., 1966). The degradation of sorbate is accompanied by the formation of 1,3-pentadiene, a volatile compound with an unpleasent hydrocarbon-like odor (Lück and Jager, 1997). Therefore, the dairy industry must rely on one preservative agent to prevent or retard the growth of mold on processed cheese. One of the greatest obstacles shredded cheese manufacturers face is product return due to mold growth (Berry, 1999). Mold growth on shredded cheese usually occurs because of the ineffective application of natamycin.

The effectiveness of natamycin to prevent or retard surface mold growth is limited by its 
ability to be evenly distributed on the food surface and its stability in the food system. In its native form, natamycin exists as an extremely insoluble crystal (Oostendorp, 1981). Each crystal imparts a zone of inhibition which successfully prevents or retards the growth of mycotic organisms. In an ideal situation, natamycin crystals will be distributed on a food surface in a manner in which all the zones of inhibition overlap. Unfortunately, this ideal situation can not be achieved because of natamycin's physical properties.

Natamycin is commonly applied to the surface of cheese by dipping or spraying, using a dispersion containing 200 to 300 ppm of the additive (CFR, 1998). Because of natamycin's relative insolubility in water, less than $5 \mathrm{ppm}$ of natamycin actually enters into true solution and is applied in a proficient manner (Schaffner and Mechlinski, 1972).

Cheese processors recognize the ineffectiveness of one application of natamycin in preventing mold growth on shredded cheese and therefore, subject shreds to more than one application of a 200 to $300 \mathrm{ppm}$ aqueous solution of natamycin. This practice is legal since the FDA only regulates the concentration at which natamycin may be applied, 200 to $300 \mathrm{ppm}$ aqueous solution. The maximum level of natamycin on the cheese surface in the U.S. is not regulated.

On December 1, 1998 the U.S. FDA amended the food additive regulations to provide for the safe use of a dry form of natamycin as an antimycotic in cheese (FR, 1999). The action was in response to a petition filed by Protein Technologies International, Inc., St. Louis, Missouri. The effectiveness of natamycin in preventing mold growth on cheese when applied in a dry form is not understood. In fact, preliminary findings reveal that the addition of $1 \%$ water / cheese (w/w) enhances natamycin's effectiveness (Suloff et al., 1999, unpublished data).

The objective of this study was to gain insight on the antimycotic action of natamycin and a natamycin semisynthetic derivative of natamycin for typical usage and storage conditions for shredded cheese. Antimycotic concentrations of 0, 10, and $20 \mathrm{ppm}$ on the cheese surface were examined. Concentrations are consistent with industry standards. Modified atmosphere packaging (MAP) and typical storage conditions were simulated. In addition, half of the samples of cheese were inoculated with Penicillium roqueforti spores.

The addition of $P$. roqueforti spores simulated heavily contaminated cheese. Initially, it 
was believed that inoculation of cheese with mold spores was needed in order to see differences between high concentrations of natamycin and derivative. Penicillium roqueforti was selected as the inoculation organisms because of its prevalence as a spoilage organism in the dairy industry. Bullerman (1981) reported that $82 \%$ of molds on refrigerated cheese belongs to the genus Penicillium. Lund et al. (1995) reported that $P$. commune, $P$. verrucosum, $P$. solitum, $P$. roqueforti, and $P$. nalgiovense accounted for most of the mold isolates in hard (e.g. Cheddar) vacuum packaged cheese. Inadequately vaccum-packed cheese showed a prevalence of $P$. roqueforti (Lund et al., 1996). Therefore, Penicillium roquefortii Thom (ATCC \#52322) isolated from a Cheddar cheese substrate in Australia was selected as the inoculum organism (ATCC, Manassas, VA).

\section{Experimental Design and Statistical Analysis}

A split-plot design was employed. There were 11 application treatments with three variables, antimycotic agent, inoculation, and antimycotic concentration. Nested within these 11 application treatments were 7 storage treatments. The experiment was repeated three times in a completely randomized block design. LSD analysis adjusted by Tukey-Kramer procedure for treatment means was performed. A 95\% confidence interval was established to detect statistical differences. Statistical analysis was performed using SAS® version 7.0 software, (SAS Institute, Inc., Cary, NC).

\section{Materials and Methods}

\section{Spore Stock Preparation}

Penicillium roquefortii Thom (ATCC \#52322) (ATCC, Manassas, VA) was cultured in Malt Extract Broth (MEB) (Difco, Detroit, MI) and then plated on Malt Extract Agar (MEA) (Difco, Detroit, MI). Conidia and ascospores were harvested by washing each plate with $10 \mathrm{ml}$

of sterile potassium phosphate : magnesium chloride diluent (PMC) $+1 \%$ Tween 80 diluent and 
gently rubbing surface with a sterile glass spreader (Nielsen et al. 1988). Suspensions were pipetted into sterile centrifuge tubes. Washing procedure was repeated two more times for a total of three washes per plate. Samples were vortexed for 2 minutes and decanted through sterile glass wool into a sterile $250 \mathrm{ml}$ flask. After thorough mixing, $25 \mathrm{ml}$ aliquots of spore suspension were transferred into sterile centrifuge tubes. Tubes were centrifuged for 10 minutes on low speed without braking (Sorvall@ Inc., RC-5B centrifuge (Newtown, CT). Supernatant was removed and $10 \mathrm{ml}$ of MEB was added to the centrifuge tubes. Mixture was then transferred to a $250 \mathrm{ml}$ sterile flask. After thorough mixing, $1 \mathrm{ml}$ spore suspensions were placed in $2 \mathrm{ml}$ cryogenic tubes and immersed in liquid nitrogen for 24 hours. Cryogenic tubes were stored in cryogenic freezer at $-80^{\circ} \mathrm{C}$ prior to lyophilization (Forma Scientific, Inc., Model 5479 freezer, Marjetta, $\mathrm{OH})$.

Spores were freeze dried in laboratory freeze dryer at $25^{\circ} \mathrm{C}$ for 24 hours to remove all water (Virtis, Gardiner, New York). The concentration of spores per gram was determined by enumerating on Dichloran Rose Bengal (DRB) Agar (Oxoid Unipath, Ogensburg, NY) supplemented with $0.1 \%$ chloramphenicol (Aldrich, Milwaukee, WI) (Ellis et al. 1993). A spore stock cellulose powder was made by adding the appropriate amount of freeze dried spores to common-type cellulose.

\section{Sample Production}

One month aged Cheddar cheese (Alto Dairy, Black Creek, WI) was shredded by a electric shredder (Paxton Corp., Shelton, CT) in $2 \mathrm{~kg}$ batches. A $2 \% \mathrm{w} / \mathrm{w}$ cellulose mixture (40 g) (Gist-brocades, Menomonee Falls, WI) was added to each of the 11 application treatments and tumbled for 2 minutes in a $110 \mathrm{~L}$ fabricated automated tumbler. The cellulose mixture in five of the application treatments was spiked with approximately $2 \times 10^{5}$ P. roqueforti spores. The addition of these spores targeted a final concentration of $10^{2} \mathrm{CFU} / \mathrm{g}$ for the shredded cheese.

During tumbling, a total of $1 \%(\mathrm{w} / \mathrm{w})$ water (20.4 g) was sprayed into the tumbler and onto the cheese. Incorporated into the $1 \%$ water spray was the appropriate concentrations of 
natamycin (Aldrich, Milwaukee, WI) or derivative. No antimycotic was added to the $1 \%$ water for 0 ppm treatments, but for 10 and 20 ppm treatments, the $1 \%$ water addition included 0.1 and $0.2 \%$ antimycotic agent.

From the $2 \mathrm{~kg}$ batch, $66 \mathrm{~g}$ samples were placed in packaging bags (Cryovac Corp., Duncan, SC). Bags were MAP using a proportional gas blender (Smith Equipment, Model 299037F, Watertown, SD) and vacuum packaging machine (Koch, Model X-200, Kansas City, MO). Gas mixture was comprised of $75 \%$ nitrogen and $25 \%$ carbon dioxide. Each package was examined for leakage by a fabricated chambered gas leak detector. Samples were stored at $4{ }^{\circ} \mathrm{C}$ in a portable walk-in refrigerator (W.H. Porter Inc. Model 1200, Holland, MI). Three replications of this experiment were conducted over a three day period.

\section{Microbiological Sampling}

Packages were opened after 0, 15, and 30 days of MAP storage. Opened packages were sampled after $0,10,20,30$, and 40 days. For each sampling bag, $33 \mathrm{~g}$ of shredded cheese was weighed and added to Fisher Scientific filtered stomacher bags (Pittsburgh, PA). A sterile $99 \mathrm{ml}$ sodium citrate diluent $(2 \% \mathrm{w} / \mathrm{v})$ was added to the stomacher bag and the sample was stomached (Seward Medical, Model 400, London, England) for 1 minute. Each sample was plated in duplicate on DRBC agar. DRBC plates were incubated at $22^{\circ} \mathrm{C}$ for 5 days (Eliot et al. 1998). Mold colonies were counted calculated, and recorded.

\section{Package Integrity and Gas Sampling}

During sample production, each package was examined for leakage by a fabricated chambered gas leak detector. Nitrogen and carbon dioxide concentrations in packages were checked immediately after packaging and again after 15 and 30 days of MAP storage by a oxygen/carbon dioxide analyzer (Illinois Instruments, Inc., Model 6600, Melrose Park, IL). 


\section{Results and Discussion}

\section{Package Integrity and Gas Analysis}

Gas analysis of MAP sample bags for replication 1, 2, and 3 showed no appreciable decrease in carbon dioxide or nitrogen. Oxygen concentration for bags tested rarely exceeded $1 \%$. The oxygen concentration for the 99 sample bags tested demonstrated a $95 \%$ confidence level of $0.36 \% \pm 0.049 \%$. These results indicate that the occurrence of mold growth in shredded cheese samples was not caused by leaker packages or improper MAP conditions.

\section{Non-inoculated Treatment Analysis}

Each sample bag was visually inspected for mold growth prior to enumeration. Results from this visual inspection revealed that sparsely spotted mold growth occurred in most samples when mold counts reached $10^{4} \mathrm{CFU} / \mathrm{g}$. For discussion purposes, it will be assumed that the product is unacceptable to the consumer when mold counts exceed $10^{4} \mathrm{CFU} / \mathrm{g}$.

Initial mold counts for non-inoculated treatments stored at 0 days MAP : 0 days open, 15 days MAP : 0 days open, and 30 days MAP : 0 days open were comparatively and statistically the same (Figure 1). MAP conditions effectively suppressed mold growth to less than $10^{1}$ $\mathrm{CFU} / \mathrm{g}$ for all non-inoculated treatments except cheese + cellulose (control) stored at 30 days MAP : 0 days open (Figure 1). Control samples had an average mold count of $10^{1} \mathrm{CFU} / \mathrm{g}$.

No statistical difference was shown between treatments stored at 0 days MAP : 10 days open and 15 days MAP : 10 days open. Mold counts for treatments stored at 0 days MAP : 10 days open ranged from nearly $10^{2}$ to less than $10^{1} \mathrm{CFU} / \mathrm{g}$ with control and cheese samples being the most heavily contaminated (Figure 1). Treatments stored at 15 days MAP : 10 days open had mold counts from slightly greater than $10^{2}$ to less than $10^{1} \mathrm{CFU} / \mathrm{g}$ with $10 \mathrm{ppm}$ and $20 \mathrm{ppm}$ natamycin treatments showing the least growth (Figure 1).

The $20 \mathrm{ppm}$ natamycin treatment stored at 30 days MAP : 10 days open was statistically different from the control, cheese, $10 \mathrm{ppm}$ and $20 \mathrm{ppm}$ derivative treatments. The $20 \mathrm{ppm}$ 
natamycin treatment was not however statistically different from the $10 \mathrm{ppm}$ natamycin treatment. Mold growth for $20 \mathrm{ppm}$ natamycin treatment stored at 30 days MAP : 10 days open was less than $10^{1} \mathrm{CFU} / \mathrm{g}$ (Figure 1). The $10 \mathrm{ppm}$ natamycin treatment exhibited a mold count of $10^{2}$ while the control and derivative treatments had mold counts from $10^{4}$ to $10^{5} \mathrm{CCU} / \mathrm{g}$ (Figure $1)$.

Statistical difference was shown between the $20 \mathrm{ppm}$ natamycin treatment and control and $10 \mathrm{ppm}$ derivative treatments stored at 0 days MAP : 20 days open. Mold counts for the 20 ppm natamycin and $20 \mathrm{ppm}$ derivative treatments were $10^{2}$ and $10^{3} \mathrm{CFU} / \mathrm{g}$, respectively (Figure 1). Cheese, control, $10 \mathrm{ppm}$ natamycin, and $10 \mathrm{ppm}$ derivative showed heavy mold growth with counts ranging from $10^{4}$ to $10^{5} \mathrm{CFU} / \mathrm{g}$ (Figure 1).

The $10 \mathrm{ppm}$ and $20 \mathrm{ppm}$ natamycin treatments and the $20 \mathrm{ppm}$ derivative treatment stored at 15 days MAP : 20 days open performed similarly and were not statistically different. The control and the $10 \mathrm{ppm}$ derivative treatment were statistically different from both the 10 ppm natamycin, $20 \mathrm{ppm}$ natamycin, and $20 \mathrm{ppm}$ derivative treatments. Mold counts for $10 \mathrm{ppm}$ natamycin, $20 \mathrm{ppm}$ natamycin and $20 \mathrm{ppm}$ derivative treatments were $10^{1}, 10^{1}$, and $10^{2} \mathrm{CFU} / \mathrm{g}$, respectively (Figure 1). Cheese, control, and $10 \mathrm{ppm}$ derivative treatments were heavily contaminated with mold counts exceeding $10^{4} \mathrm{CFU} / \mathrm{g}$ (Figure 1).

The $10 \mathrm{ppm}$ and $20 \mathrm{ppm}$ natamycin treatments stored at 30 days MAP : 20 days open performed similarly in suppressing mold growth to $10^{3} \mathrm{CFU} / \mathrm{g}$ (Figure 1). These treatments were statistically different from cheese, control, and $10 \mathrm{ppm}$ derivative treatments. Although not statistically different from the $10 \mathrm{ppm}$ and $20 \mathrm{ppm}$ natamycin treatments, the $20 \mathrm{ppm}$ derivative treatment was heavily contaminated with a mold count exceeding $10^{4} \mathrm{CFU} / \mathrm{g}$ (Figure 1).

All treatments stored at 0 days MAP : 30 days open were heavily contaminated with mold counts exceeding $10^{6} \mathrm{CFU} / \mathrm{g}$ (Figure 1). No statistical difference was shown between any of the treatments. The $20 \mathrm{ppm}$ natamycin treatment was the most effective treatment in retarding mold growth for storage treatments: 15 days MAP : 30 days open and 30 days MAP : 30 days open. The $20 \mathrm{ppm}$ natamycin treatment retarded mold growth to $10^{3} \mathrm{CFU} / \mathrm{g}$ for both storage conditions (Figure 1). All other treatments stored under those conditions had mold counts exceeding $10^{5} \mathrm{CFU} / \mathrm{g}$ (Figure 1). Even though the $20 \mathrm{ppm}$ natamycin treatment was 
comparatively different from all other treatments for these storage conditions, statistical differences were only found between the $20 \mathrm{ppm}$ treatments and the two control treatments.

For storage treatments, 0 days MAP : 40 days open, 15 days MAP : 40 days open, and 30 days MAP : 40 days open there were no statistical differences between any of the treatments. All samples were heavily contaminated with mold counts exceeding $10^{4} \mathrm{CFU} / \mathrm{g}$ (Figure 1).

Statistical differences of mold counts for treatments stored under the same conditions were not indicative of practical microbiological concerns concerning spoilage in many instances. LSD analysis adjusted by the Tukey-Kramer procedure for treatment means was performed as part of the statistical analysis. The Tukey-Kramer procedure is one of the most conservative procedures in statistics for calculating confidence intervals. Therefore, it is possible that this procedure would indicate no statistical difference between treatments when one may exist in a practical sense. The transit time required for samples to exceed mold counts of $10^{4} \mathrm{CFU} / \mathrm{g}$ is a better benchmark to examine the effectiveness of each treatment.

Statistical analysis of main treatment effects revealed several important conclusions. The 10 ppm natamycin, 20 ppm natamycin, and 20 ppm derivative treatments were statistically different from control. These differences show that these treatments were effective in retarding mold growth in shredded Cheddar cheese. No statistical difference was found between control and the $10 \mathrm{ppm}$ derivative treatment. The $10 \mathrm{ppm}$ derivative treatment was ineffective.

No statistical difference occurred between $10 \mathrm{ppm}$ and $20 \mathrm{ppm}$ natamycin. Also, no statistical difference was found between $10 \mathrm{ppm}$ natamycin and $20 \mathrm{ppm}$ derivative. These treatments can be used interchangeable with one another with no noticeable change in mold growth. Although no statistical difference was found between $10 \mathrm{ppm}$ and $20 \mathrm{ppm}$ natamycin treatments, there is a statistical difference between 20 ppm natamycin and 20 ppm derivative.

\section{Inoculated Treatment Analysis}

The inoculated portion of this experiment did not reveal any meaningful results. No statistical differences were found between inoculated control and antibiotic treatments. Mold counts exceeded $10^{4} \mathrm{CFU} / \mathrm{g}$ for all treatments after 10 days open. 


\section{Storage Condition Analysis}

Statistical analysis of main storage condition effects were performed in a similar manner to main treatment effects. No statistical differences in mold counts were found between 0 days MAP, 15 days MAP, and 30 days MAP for packages at 0 days open. Statistical differences were found between 0 days MAP and 30 days MAP as well as between 15 days MAP and 30 days MAP at 10 days open. No statistical difference was found between 0 days MAP and 15 days MAP for 10 day open packages. These results indicate that mold growth is much more rapid after packages have been stored in MA conditions longer than 15 days.

Results from 20 day open packages confirm this statement. No statistical difference was found between 0 day MAP and 15 day MAP packages after 20 days open, but statistical differences were found between samples stored at 0 day MAP and 30 day MAP as well as 15 day MAP and 30 day MAP after 20 days open. No statistical differences were found between storage conditions after 30 days open. Forty day open samples are not meaningful to discuss.

\section{Conclusions}

Natamycin was more effective than the derivative in retarding mold growth in one month aged shredded Cheddar cheese at every concentration and storage condition. The application of 20 ppm natamycin effectively retarded mold growth below $10^{4} \mathrm{CFU} / \mathrm{g}$ for samples stored at 15 and 30 days MAP and opened for as long as 30 days. Visual mold growth in most samples occurred after mold counts exceeded $10^{4} \mathrm{CFU} / \mathrm{g}$. Although the $20 \mathrm{ppm}$ natamycin treatment was not statistically different from the $10 \mathrm{ppm}$ natamycin treatment, it was different in preventing visible mold growth on the shreds. The $20 \mathrm{ppm}$ natamycin treatment was the most effective treatment in preventing mold growth.

The 10 ppm natamycin and 20 ppm derivative performed similarly. No statistical or comparative differences were found between these two treatments. Mold counts did not exceed $10^{4} \mathrm{CFU} / \mathrm{g}$ for samples opened as long as 20 days for all MAP storage conditions. Cheese, 
control, and the $10 \mathrm{ppm}$ derivative treatment showed visible mold growth in most samples at 20 days open and were noticeably ineffective at suppressing mold growth.

Unfortunately, the inoculated portion of the study did not reveal any meaningful results. No differences were found for any of the antibiotic treatments. Analysis of storage conditions revealed an important observation about MAP storage. The outgrowth of mold in shredded Cheddar cheese stored in MAP conditions longer than 15 days is much greater than samples stored under those conditions less than 15 days. This bloom in mold growth may occur as a result of the degradation of natamycin and the derivative throughout storage.

The development of semisynthetic derivatives of natamycin should continue. Even though the semisynthetic derivative tested in this study was less effective in preventing mold growth in processed shredded Cheddar cheese it still maintained a substantial degree of efficacy. In addition, the derivative may have processing advantages due to its increased water solubility. The chance of spray nozzles becoming clogged during application is less likely with the derivative then natamycin. 
Tables

Table 1: Mold growth on 1 month aged shredded Cheddar cheese for non-inoculated treatments at different storage conditions and antibiotic treatments

\begin{tabular}{|c|c|c|c|c|c|c|c|}
\hline \multicolumn{2}{|l|}{ Storage Conditions } & Cheese & $\begin{array}{c}\text { Control: } \\
\text { Cheese }+ \\
\text { Cellulose }\end{array}$ & $\begin{array}{c}10 \text { ppm } \\
\text { Natamycin }\end{array}$ & $\begin{array}{c}10 \text { ppm } \\
\text { Derivative }\end{array}$ & $\begin{array}{c}20 \text { ppm } \\
\text { Natamycin }\end{array}$ & $\begin{array}{c}20 \text { ppm } \\
\text { Derivative }\end{array}$ \\
\hline $\begin{array}{c}\text { Days } \\
\text { MAP }\end{array}$ & $\begin{array}{c}\text { Days } \\
\text { Open }\end{array}$ & $\begin{array}{c}\text { Mold Growth } \\
\text { Log (CFU/g) }\end{array}$ & $\begin{array}{c}\text { Mold Growth } \\
\text { Log (CFU/g) }\end{array}$ & Mold Growth & Mold Growth & Mold Growth $)$ & Mold Growth \\
Log (CFU/g) & Log (CFU/g) & Log (CFU/g) \\
\hline 0 & 0 & 0.60 & 0.60 & 0.60 & 0.60 & 0.60 & 0.60 \\
\hline 0 & 10 & 1.98 & 1.69 & 0.60 & 0.60 & 0.96 & 0.60 \\
\hline 0 & 20 & 4.26 & 5.98 & 4.45 & 5.27 & 2.00 & 3.29 \\
\hline 0 & 30 & 6.80 & 8.42 & 6.68 & 6.99 & 6.06 & 6.44 \\
\hline 0 & 40 & 7.48 & 8.70 & 7.43 & 8.14 & 7.01 & 7.68 \\
\hline 15 & 0 & 0.60 & 0.60 & 0.60 & 0.60 & 0.60 & 0.60 \\
\hline 15 & 10 & 1.15 & 1.44 & 0.60 & 2.07 & 0.60 & 1.16 \\
\hline 15 & 20 & 4.55 & 5.75 & 1.62 & 5.58 & 1.42 & 2.13 \\
\hline 15 & 30 & 6.50 & 7.75 & 5.95 & 6.78 & 3.86 & 5.76 \\
\hline 15 & 40 & 6.89 & 7.12 & 6.72 & 7.18 & 4.63 & 5.09 \\
\hline 30 & 0 & 0.60 & 1.51 & 0.60 & 0.90 & 0.60 & 0.60 \\
\hline 30 & 10 & 4.87 & 5.10 & 2.22 & 4.16 & 0.60 & 4.32 \\
\hline 30 & 20 & 6.87 & 7.02 & 2.41 & 6.36 & 2.16 & 4.35 \\
\hline 30 & 30 & 7.52 & 7.89 & 5.51 & 7.95 & 3.45 & 6.42 \\
\hline 30 & 40 & 8.57 & 8.19 & 5.46 & 8.08 & 6.83 & 7.19 \\
\hline
\end{tabular}




\section{References}

Berry, D. 1999. Natamycin for shredded cheese. Dairy Foods. 100:45.

CFR, 1998. Code of Federal Regulations. Title 21. Part 172. Government Printing Office, Washington, DC.

Eliot, S.C., Vuillemard, J.C., and Emond, J.P. 1998. Stability of shredded Mozzarella cheese under modified atmospheres. J. Food Sci. 63:1075-1080.

Ellis, W.O., Smith, J.P., Simpson, B.K., and Ramaswamy, H. 1993. Effect of inoculum level on aflatoxin production by Aspergillus flavus under modified atmosphere packaging (MAP) conditions. Food Microbiol. 10:9-12.

Finol, M.L., Marth, E.H., and Lindsay, R.C. 1982. Depletion of sorbate from different media during growth of Penicillium species. J. Food Protect. 45:398-404.

FR, 1998. Federal Register. Vol. 63. p. 66,014. Government Printing Office, Washington, DC.

Lück, E. and Jager, M. 1997. Antimicrobial Food Additives, $2^{\text {nd }}$ ed. Springer-Verlag, New York, p. 152-167.

Marth, E.H., Capp, C.M., Hasenzahl, L., Jackson, H.W., and Hussong, R.V. 1966. Degradation of potassium sorbate by Penicillium species. J. Dairy Sci. 49:1197-1205.

Nielsen, R.V., Beuchat, L.R., and Frisvad, J.C. 1988. Growth of and fumitremorgin production by Neosartorya fischeri as affected by temperature, light, and water activity. Appl. Environ. Microbiol. 54:1504-1510. 
Oostendorp, J.G. 1981. Natamycin. Antonie van Leeuwenhoek. 47:170-171.

Schaffner, C.P. and Mechlinski, W. 1972. Polyene macrolide derivatives. II. Physical-chemical properties of polyene macrolide esters and their water soluble salts. J. Antibiotics. 25:259-260.

Sofos, J.N. and Busta, F.F. 1981. Antimicrobial activity of sorbate. J. Food Protect. 44:614-622.

Suloff, E.C., Marcy, J.E. and Yaun, B. 1999. Unpublished data. Dept. of Food Science and Technology, Virginia Polytechnic Institute and State University, Blacksburg, VA. 


\section{Appendix}

Table 1: Application treatments for microbiological challenge study of semisynthetic derivative of natamycin and parent antibiotic

\begin{tabular}{|c|c|c|}
\hline Application Treatment (1) & Inoculation (2) & $\begin{array}{c}\text { Antibiotic } \\
\text { Concentration }\end{array}$ \\
\hline None & Negative & $0 \mathrm{ppm}$ \\
\hline Common-type cellulose & Negative & $0 \mathrm{ppm}$ \\
\hline Common-type cellulose + natamycin & Negative & $10 \mathrm{ppm}$ \\
\hline Common-type cellulose + derivative & Negative & $10 \mathrm{ppm}$ \\
\hline Common-type cellulose + natamycin & Negative & $20 \mathrm{ppm}$ \\
\hline Common-type cellulose + derivative & Negative & $20 \mathrm{ppm}$ \\
\hline Common-type cellulose & Positive & $0 \mathrm{ppm}$ \\
\hline Common-type cellulose + natamycin & Positive & $10 \mathrm{ppm}$ \\
\hline Common-type cellulose + derivative & Positive & $10 \mathrm{ppm}$ \\
\hline Common-type cellulose + natamycin & Positive & $20 \mathrm{ppm}$ \\
\hline Common-type cellulose + derivative & Positive & $20 \mathrm{ppm}$ \\
\hline $\begin{array}{l}\text { (1) Applications with common-type cellulose contain 2\%(w/w) cellulose mixture } \\
\text { (2) Inoculation with Penicilium roqueforti spores }\end{array}$ & \\
\hline \multicolumn{2}{|l}{} \\
\hline
\end{tabular}

Table 2: Storage treatments for microbiological challenge study of semisynthetic derivative of natamycin and parent antibiotic

\begin{tabular}{|c|}
\hline Storage Treatment \\
\hline 0 Days MAP / 0 Days Opened \\
\hline 0 Days MAP / 10 Days Opened \\
\hline 0 Days MAP / 20 Days Opened \\
\hline 0 Days MAP / 30 Days Opened \\
\hline 0 Days MAP / 40 Days Opened \\
\hline 15 Days MAP / 0 Days Opened \\
\hline 15 Days MAP / 10 Days Opened \\
\hline 15 Days MAP / 20 Days Opened \\
\hline 15 Days MAP / 30 Days Opened \\
\hline 15 Days MAP / 40 Days Opened \\
\hline 30 Days MAP / 0 Days Opened \\
\hline 30 Days MAP / 10 Days Opened \\
\hline 30 Days MAP / 20 Days Opened \\
\hline 30 Days MAP / 30 Days Opened \\
\hline 30 Days MAP / 40 Days Opened \\
\hline
\end{tabular}


Figure 1: Statistical model for microbiological challenge study of semisynthetic derivative of natamycin and parent antibiotic

$$
\begin{aligned}
& Y_{i j k}=m+R_{i}+A_{j}+\varepsilon_{i j}^{A}+B_{k}+(A B)_{j k}+\varepsilon^{B}{ }_{i j k} \\
& \mathrm{i}=\text { replication } \\
& \mathrm{j}=\text { factor } \mathrm{A} \\
& \mathrm{k}=\text { factor } \mathrm{B} \\
& \text { where } \mu=\text { overall mean } \\
& \mathrm{R}_{\mathrm{i}}=\text { replicate effect } \\
& A_{j}=\text { effect of } j^{\text {th }} \text { level of factor } A \\
& B_{k}=\text { effect of } k^{\text {th }} \text { level of factor } B \\
& (\mathrm{AB})_{\mathrm{jk}}=\text { interaction } \\
& \varepsilon^{\mathrm{A}} \mathrm{ij}=\text { error } \mathrm{A} \\
& \varepsilon^{\mathrm{B}}{ }_{\mathrm{ijk}}=\operatorname{error} \mathrm{B}
\end{aligned}
$$


Table 4: Gas analysis of MAP sample bags for replication 1

\begin{tabular}{|c|c|c|c|c|c|c|}
\hline Gas Analyzed From: & Inoculation & Replication & Days MAP & $\mathrm{CO}_{2}(\%)$ & $\mathrm{O}_{2}(\%)$ & $\mathrm{N}_{2}(\%)$ \\
\hline Mixer & NA & 1 & 0 & 30.00 & 0.05 & 69.95 \\
\hline Mixer & NA & 1 & 0 & 29.90 & 0.05 & 70.05 \\
\hline Control 1: Cheese & - & 1 & 0 & 28.80 & 0.21 & 70.99 \\
\hline Control 2: Cheese + Cellulose & - & 1 & 0 & 28.30 & 0.37 & 71.33 \\
\hline 10 ppm Natamycin & - & 1 & 0 & 26.30 & 0.20 & 73.50 \\
\hline 10 ppm Derivative & - & 1 & 0 & 28.90 & 0.10 & 71.00 \\
\hline 20 ppm Natamycin & - & 1 & 0 & 27.10 & 0.21 & 72.69 \\
\hline 20 ppm Derivative & - & 1 & 0 & 27.00 & 0.16 & 72.84 \\
\hline Control 3: Cheese + Cellulose & + & 1 & 0 & 27.60 & 0.13 & 72.27 \\
\hline 10 ppm Natamycin & + & 1 & 0 & 27.50 & 0.17 & 72.34 \\
\hline 10 ppm Derivative & + & 1 & 0 & 27.80 & 0.22 & 71.98 \\
\hline 20 ppm Natamycin & + & 1 & 0 & 27.70 & 0.14 & 72.16 \\
\hline 20 ppm Derivative & + & 1 & 0 & 28.20 & 0.36 & 71.44 \\
\hline Control 1: Cheese & - & 1 & 15 & 26.40 & 0.41 & 73.19 \\
\hline Control 2: Cheese + Cellulose & - & 1 & 15 & 24.80 & 0.55 & 74.66 \\
\hline 10 ppm Natamycin & - & 1 & 15 & 24.80 & 0.50 & 74.70 \\
\hline 10 ppm Derivative & - & 1 & 15 & 25.20 & 0.55 & 74.25 \\
\hline 20 ppm Natamycin & - & 1 & 15 & 25.70 & 0.47 & 73.83 \\
\hline 20 ppm Derivative & - & 1 & 15 & 24.90 & 0.48 & 74.62 \\
\hline Control 3: Cheese + Cellulose & + & 1 & 15 & 25.00 & 0.43 & 74.57 \\
\hline 10 ppm Natamycin & + & 1 & 15 & 25.40 & 0.41 & 74.19 \\
\hline 10 ppm Derivative & + & 1 & 15 & 25.40 & 0.46 & 74.14 \\
\hline 20 ppm Natamycin & + & 1 & 15 & 25.10 & 0.46 & 74.44 \\
\hline 20 ppm Derivative & + & 1 & 15 & 25.10 & 0.50 & 74.40 \\
\hline Control 1: Cheese & - & 1 & 30 & 24.80 & 0.71 & 74.49 \\
\hline Control 2: Cheese + Cellulose & - & 1 & 30 & 23.00 & 0.86 & 76.15 \\
\hline 10 ppm Natamycin & - & 1 & 30 & 23.70 & 0.77 & 75.54 \\
\hline 10 ppm Derivative & - & 1 & 30 & 24.50 & 0.72 & 74.78 \\
\hline 20 ppm Natamycin & - & 1 & 30 & 24.70 & 0.67 & 74.63 \\
\hline 20 ppm Derivative & - & 1 & 30 & 23.70 & 0.69 & 75.61 \\
\hline Control 3: Cheese + Cellulose & + & 1 & 30 & 24.70 & 0.08 & 75.23 \\
\hline 10 ppm Natamycin & + & 1 & 30 & 24.60 & 0.61 & 74.79 \\
\hline 10 ppm Derivative & + & 1 & 30 & 24.80 & 0.09 & 75.11 \\
\hline 20 ppm Natamycin & + & 1 & 30 & 24.40 & 0.66 & 74.94 \\
\hline 20 ppm Derivative & + & 1 & 30 & 25.00 & 0.22 & 74.78 \\
\hline
\end{tabular}


Table 5: Gas analysis of MAP sample bags for replication 2

\begin{tabular}{|c|c|c|c|c|c|c|}
\hline Gas Analyzed From: & Inoculation & Replication & Days MAP & $\mathrm{CO}_{2}(\%)$ & $\mathrm{O}_{2}(\%)$ & $\mathrm{N}_{2}(\%)$ \\
\hline Mixer & NA & 2 & 0 & 29.40 & 0.01 & 70.59 \\
\hline Control 1: Cheese & - & 2 & 0 & 28.00 & 0.12 & 71.88 \\
\hline Control 2: Cheese + Cellulose & - & 2 & 0 & 29.00 & 0.10 & 70.90 \\
\hline 10 ppm Natamycin & - & 2 & 0 & 29.00 & 0.11 & 70.89 \\
\hline 10 ppm Derivative & - & 2 & 0 & 27.30 & 0.25 & 72.46 \\
\hline 20 ppm Natamycin & - & 2 & 0 & 27.70 & 0.17 & 72.13 \\
\hline 20 ppm Derivative & - & 2 & 0 & 27.80 & 0.21 & 72.00 \\
\hline Control 3: Cheese + Cellulose & + & 2 & 0 & 28.00 & 0.20 & 71.80 \\
\hline 10 ppm Natamycin & + & 2 & 0 & 28.20 & 0.14 & 71.66 \\
\hline 10 ppm Derivative & + & 2 & 0 & 28.10 & 0.17 & 71.73 \\
\hline 20 ppm Natamycin & + & 2 & 0 & 27.80 & 0.18 & 72.02 \\
\hline 20 ppm Derivative & + & 2 & 0 & 28.30 & 0.17 & 71.53 \\
\hline Control 1: Cheese & - & 2 & 15 & 25.80 & 0.38 & 73.83 \\
\hline Control 2: Cheese + Cellulose & - & 2 & 15 & 26.20 & 0.39 & 73.41 \\
\hline 10 ppm Natamycin & - & 2 & 15 & 25.50 & 0.40 & 74.10 \\
\hline 10 ppm Derivative & - & 2 & 15 & 24.50 & 0.53 & 74.97 \\
\hline 20 ppm Natamycin & - & 2 & 15 & 25.00 & 0.44 & 74.56 \\
\hline 20 ppm Derivative & - & 2 & 15 & 25.50 & 0.39 & 74.11 \\
\hline Control 3: Cheese + Cellulose & + & 2 & 15 & 26.00 & 0.15 & 73.85 \\
\hline 10 ppm Natamycin & + & 2 & 15 & 25.80 & 0.33 & 73.87 \\
\hline 10 ppm Derivative & + & 2 & 15 & 26.10 & 0.19 & 73.71 \\
\hline 20 ppm Natamycin & + & 2 & 15 & 25.70 & 0.34 & 73.96 \\
\hline 20 ppm Derivative & + & 2 & 15 & 25.50 & 0.28 & 74.22 \\
\hline Control 1: Cheese & - & 2 & 30 & 24.90 & 0.54 & 74.57 \\
\hline Control 2: Cheese + Cellulose & - & 2 & 30 & 25.50 & 0.52 & 73.98 \\
\hline 10 ppm Natamycin & - & 2 & 30 & 25.10 & 0.63 & 74.27 \\
\hline 10 ppm Derivative & - & 2 & 30 & 24.30 & 0.68 & 75.02 \\
\hline 20 ppm Natamycin & - & 2 & 30 & 25.40 & 0.54 & 74.06 \\
\hline 20 ppm Derivative & - & 2 & 30 & 24.60 & 0.61 & 74.79 \\
\hline Control 3: Cheese + Cellulose & + & 2 & 30 & 25.40 & 0.08 & 74.53 \\
\hline 10 ppm Natamycin & + & 2 & 30 & 25.70 & 0.32 & 73.98 \\
\hline 10 ppm Derivative & + & 2 & 30 & 25.70 & 0.06 & 74.24 \\
\hline 20 ppm Natamycin & + & 2 & 30 & 25.20 & 0.44 & 74.36 \\
\hline 20 ppm Derivative & + & 2 & 30 & 25.40 & 0.05 & 74.55 \\
\hline
\end{tabular}


Table 6: Gas analysis of MAP sample bags for replication 3

\begin{tabular}{|c|c|c|c|c|c|c|}
\hline Gas Analyzed From: & Inoculation & Replication & Days MAP & $\mathrm{CO}_{2}(\%)$ & $\mathrm{O}_{2}(\%)$ & $\mathrm{N}_{2}(\%)$ \\
\hline Mixer & NA & 3 & 0 & 29.70 & 0.03 & 70.27 \\
\hline Control 1: Cheese & - & 3 & 0 & 28.40 & 0.18 & 71.42 \\
\hline Control 2: Cheese + Cellulose & - & 3 & 0 & 28.30 & 0.10 & 71.60 \\
\hline 10 ppm Natamycin & - & 3 & 0 & 28.10 & 0.10 & 71.80 \\
\hline 10 ppm Derivative & - & 3 & 0 & 28.10 & 0.13 & 71.77 \\
\hline 20 ppm Natamycin & - & 3 & 0 & 28.10 & 0.16 & 71.74 \\
\hline 20 ppm Derivative & - & 3 & 0 & 28.20 & 0.14 & 71.66 \\
\hline Control 3: Cheese + Cellulose & + & 3 & 0 & 28.20 & 0.11 & 71.69 \\
\hline 10 ppm Natamycin & + & 3 & 0 & 28.10 & 0.08 & 71.82 \\
\hline 10 ppm Derivative & + & 3 & 0 & 28.40 & 0.07 & 71.53 \\
\hline 20 ppm Natamycin & + & 3 & 0 & 28.50 & 0.09 & 71.41 \\
\hline 20 ppm Derivative & + & 3 & 0 & 28.60 & 0.07 & 71.34 \\
\hline Control 1: Cheese & - & 3 & 15 & 25.90 & 0.43 & 73.67 \\
\hline Control 2: Cheese + Cellulose & - & 3 & 15 & 26.20 & 0.45 & 73.35 \\
\hline 10 ppm Natamycin & - & 3 & 15 & 26.40 & 0.41 & 73.19 \\
\hline 10 ppm Derivative & - & 3 & 15 & 26.10 & 1.22 & 72.68 \\
\hline 20 ppm Natamycin & - & 3 & 15 & 26.60 & 0.37 & 73.03 \\
\hline 20 ppm Derivative & - & 3 & 15 & 26.20 & 1.20 & 72.60 \\
\hline Control 3: Cheese + Cellulose & + & 3 & 15 & 26.40 & 1.07 & 72.53 \\
\hline 10 ppm Natamycin & + & 3 & 15 & 27.40 & 0.37 & 72.23 \\
\hline 10 ppm Derivative & + & 3 & 15 & 26.70 & 0.30 & 73.00 \\
\hline 20 ppm Natamycin & + & 3 & 15 & 26.90 & 0.36 & 72.74 \\
\hline 20 ppm Derivative & + & 3 & 15 & 26.40 & 0.32 & 73.28 \\
\hline Control 1: Cheese & - & 3 & 30 & 25.90 & 0.59 & 73.52 \\
\hline Control 2: Cheese + Cellulose & - & 3 & 30 & 24.40 & 0.74 & 74.86 \\
\hline 10 ppm Natamycin & - & 3 & 30 & 25.90 & 0.54 & 73.56 \\
\hline 10 ppm Derivative & - & 3 & 30 & 25.50 & 0.56 & 73.94 \\
\hline 20 ppm Natamycin & - & 3 & 30 & 25.30 & 0.61 & 74.09 \\
\hline 20 ppm Derivative & - & 3 & 30 & 26.60 & 0.45 & 72.95 \\
\hline Control 3: Cheese + Cellulose & + & 3 & 30 & 27.00 & 0.05 & 72.95 \\
\hline 10 ppm Natamycin & + & 3 & 30 & 27.30 & 0.23 & 72.47 \\
\hline 10 ppm Derivative & + & 3 & 30 & 25.70 & 0.05 & 74.25 \\
\hline 20 ppm Natamycin & + & 3 & 30 & 26.20 & 0.51 & 73.29 \\
\hline 20 ppm Derivative & + & 3 & 30 & 26.90 & 0.11 & 72.99 \\
\hline
\end{tabular}


Figure 2: Mold growth on 1 month aged shredded Cheddar cheese for antibiotic treatments vs. storage conditions: 0 days $M A P$ : 0, 10, 20, 30, 40 days open

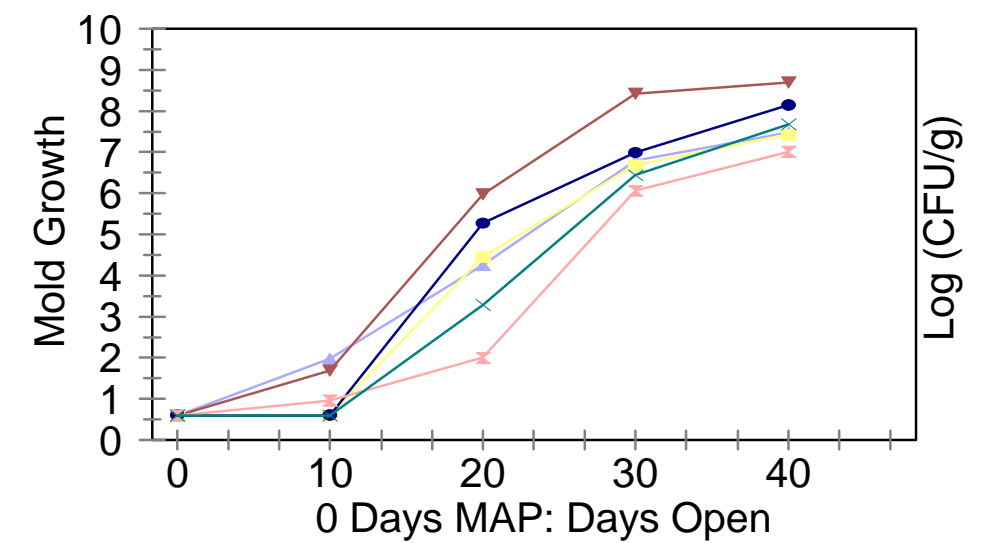

\begin{tabular}{ll}
$\rightarrow$ Cheese & $\rightarrow$ Control: Cheese + Cellulose \\
-10 ppm Natamycin & $\rightarrow 10$ ppm Derivative \\
-20 ppm Natamycin & $-\leftarrow 20$ ppm Derivative \\
\hline
\end{tabular}

Figure 3: Mold growth on 1 month aged shredded Cheddar cheese for antibiotic treatments vs. storage conditions: 15 days MAP : 0, 10, 20, 30, 40 days open

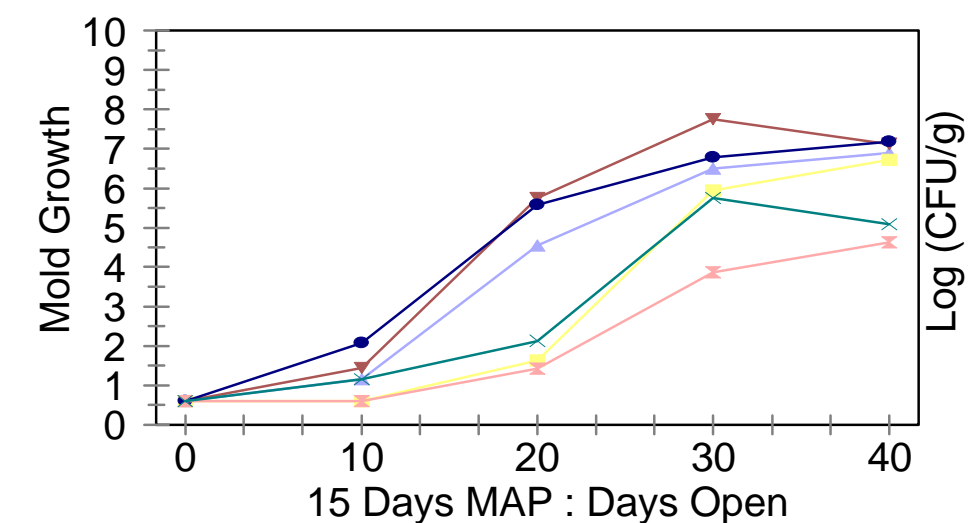

\begin{tabular}{ll|}
$\rightarrow-$ Cheese & $\rightarrow$ Control: Cheese + Cellulose \\
-10 ppm Natamycin & $\rightarrow 10$ ppm Derivative \\
-20 ppm Natamycin & $\rightarrow-20$ ppm Derivative \\
\hline
\end{tabular}


Figure 4: Mold growth on 1 month aged shredded Cheddar cheese for antibiotic treatments vs. storage conditions: 30 days MAP : 0, 10, 20, 30, 40 days open

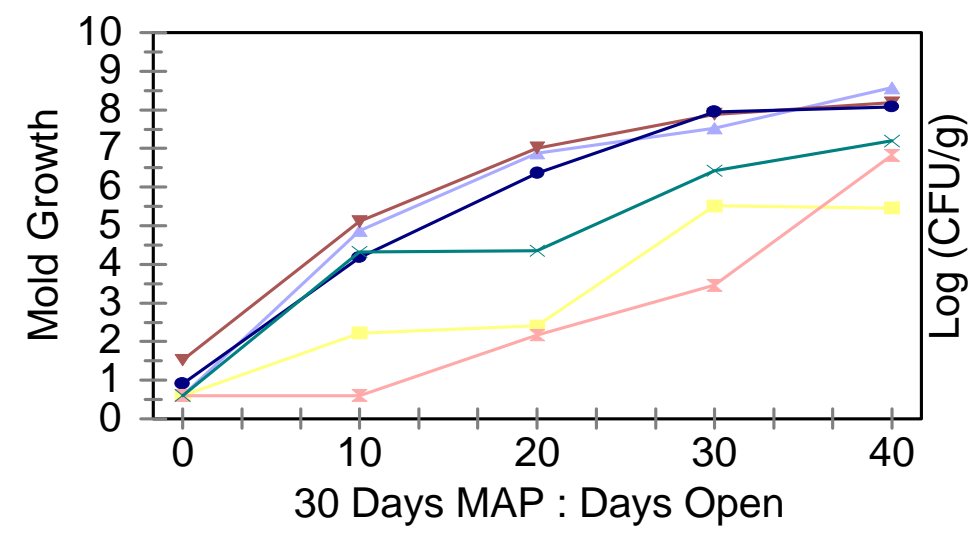

\begin{tabular}{rl|}
$\rightarrow-$ Control 1: Cheese & $\rightarrow$ Control 2: Cheese + Cellulose \\
-10 ppm Natamycin & $\rightarrow-10$ ppm Derivative \\
-20 ppm Natamycin & $* 20$ ppm Derivative
\end{tabular}


Table 7: Mold growth on 1 month ripened shredded Cheddar cheese inoculated with Penicillium roqueforti spores at various storage conditions and antibiotic treatments

\begin{tabular}{|c|c|c|c|c|c|c|}
\hline \multicolumn{2}{|l|}{ Storage Conditions } & \begin{tabular}{c} 
Control: \\
Inoculated \\
Cheese + \\
\multicolumn{2}{|c|}{}
\end{tabular} & \begin{tabular}{c}
10 ppm \\
Natamycin \\
\multicolumn{2}{|c|}{}
\end{tabular} & $\begin{array}{c}10 \text { ppm } \\
\text { Derivative }\end{array}$ & $\begin{array}{c}20 \text { ppm } \\
\text { Natamycin }\end{array}$ & $\begin{array}{c}20 \text { ppm } \\
\text { Derivative }\end{array}$ \\
\hline $\begin{array}{c}\text { Days } \\
\text { MAP }\end{array}$ & Days Open & $\begin{array}{c}\text { Mold Growth } \\
\text { Log (CFU/g) }\end{array}$ & $\begin{array}{c}\text { Mold Growth } \\
\text { Log (CFU/g) }\end{array}$ & $\begin{array}{c}\text { Mold Growth } \\
\text { Log (CFU/g) }\end{array}$ & $\begin{array}{c}\text { Mold Growth } \\
\text { Log (CFU/g) }\end{array}$ & $\begin{array}{c}\text { Mold Growth } \\
\text { Log (CFU/g) }\end{array}$ \\
\hline 0 & 0 & 3.05 & 3.05 & 3.11 & 3.05 & 3.07 \\
\hline 0 & 10 & 4.31 & 3.66 & 3.86 & 3.19 & 3.89 \\
\hline 0 & 20 & 5.95 & 5.82 & 6.02 & 5.29 & 6.20 \\
\hline 0 & 30 & 6.74 & 6.74 & 6.39 & 6.35 & 6.66 \\
\hline 0 & 40 & 6.78 & 7.36 & 6.72 & 7.08 & 6.67 \\
\hline 15 & 0 & 2.82 & 2.84 & 2.91 & 2.81 & 2.80 \\
\hline 15 & 10 & 5.29 & 4.28 & 5.24 & 3.63 & 5.06 \\
\hline 15 & 20 & 6.24 & 6.09 & 6.37 & 5.11 & 6.24 \\
\hline 15 & 30 & 6.50 & 6.49 & 7.11 & 6.32 & 6.79 \\
\hline 15 & 40 & 7.52 & 7.09 & 7.83 & 7.36 & 7.23 \\
\hline 30 & 0 & 3.20 & 3.77 & 3.44 & 3.22 & 3.68 \\
\hline 30 & 10 & 5.90 & 5.88 & 6.35 & 4.63 & 5.97 \\
\hline 30 & 20 & 7.35 & 7.24 & 7.58 & 6.07 & 7.15 \\
\hline 30 & 30 & 8.14 & 7.84 & 7.63 & 7.13 & 7.73 \\
\hline 30 & 40 & 8.23 & 7.90 & 8.11 & 7.53 & 7.84 \\
\hline
\end{tabular}




\title{
CHAPTER 4: STABILITY CHALLENGE STUDY FOR SEMISYNTHETIC DERIVATIVE OF NATAMYCIN AND PARENT ANTIBIOTIC
}

\begin{abstract}
A comparative study investigating the stability of a semisynthetic derivative of natamycin and the parent antibiotic on one month aged shredded Cheddar cheese MAP packaged was performed. Antibiotic concentration on the cheese was quantified by molecular absorption spectroscopy (IDF, 1992). Heavily contaminated samples caused the rate and loss of natamycin and the derivative to increase. Antibiotic concentration decreased at a nearly identical rate in MAP and open package conditions. Natamycin and derivative have similar degradation properties.
\end{abstract}

\section{Introduction}

The effectiveness of natamycin to prevent or retard mold growth is limited to its ability to be evenly distributed on the food surface and its stability in the food system. In its native form, natamycin exists as an extremely insoluble crystal (Oostendorp, 1981). Each crystal imparts a zone of inhibition which successfully prevents or retards the growth of mycotic organisms. It is not however known whether the crystalline state of natamycin is necessary for activity. Physical and chemical conditions which effect natamycin's stability are well documented, but how these conditions effect natamycin in a food system have not been previously studied. This study investigated the stability of natamycin and a semisynthetic derivative of natamycin on one month aged shredded Cheddar cheese modified atmosphere packaged (MAP).

Exposure to ultraviolet light and chemical oxidation are the two most important degradation factors for natamycin. Ultraviolet light with wavelengths of 300 to $350 \mathrm{~nm}$ quickly inactivates natamycin (Dekker and Ark, 1959). The tetraene structure of natamycin causes its instability in the presence ultraviolet light. The photodynamic destruction of natamycin occurs 
by a unique triplet-triplet transfer mechanism. Most light induced destructions occur as a result of oxidation or by a free-radical mechanism. A triplet-triplet transfer occurs when a compound in an excited triplet state collides with a compound in the singlet state at a lower energy state. The result is a conversion of spin movement for the singlet state compound causing it to excite the molecule. An experimental demonstration of this phenomenon was conducted for natamycin. Excited riboflavin molecules in the triplet state bombarded natamycin in the 300 to $350 \mathrm{~nm}$ range causing a triplet-triplet transfer. The triplet-triplet transfer caused the natamycin molecule to quickly degrade the reactive tetraene structure (Posthuma and Berends 1960 and 1961). Photodynamic destruction of natamycin caused by triplet-triplet transfer does not show decreases in maxima peaks 279, 290,304, and $318 \mathrm{~nm}$ in an ultraviolet absorption spectra (Thomas 1976).

Natamycin suspensions and solutions lose their biological activity by oxidation. Oxidation is promoted by elevated temperatures, greater than $37^{\circ} \mathrm{C}$, and in the presence of chemical oxidants (Dekker and Ark 1959 and Barr 1959). Chemical oxidants such as peroxides, perchlorates, persulfates, permanganates, iodates, bromates, hypochlorites, sulfites, and anhydrides of organic acids cause the oxidative inactivation of natamycin. The ultraviolet absorption spectra of natamycin following oxidative inactivation show marked decreases in maxima peaks, 279, 290, 304, and $318 \mathrm{~nm}$ (Thomas 1976).

Oxidation is amplified at pH levels below 5.0 and above 9.0 (Raab 1972). In organic acids, acid hydrolysis occurs resulting in the elimination of the mycosamine. Natamycin changes its structure from a tetraene to a pentaene as a result (Thomas 1976). Oxidative inactivation may be prevented by the addition of chlorophyll, ascorbic acid, or a number of other antioxidants (Dekker and Ark 1959 and Barr 1959).

The stability of natamycin and derivative on one month aged shredded Cheddar cheese was investigated. Antibiotic treatments of 10 and $20 \mathrm{ppm}$ on the cheese surface were examined. Concentrations are consistent with industry standards. MAP and typical storage conditions were simulated. In addition, half of the samples of cheese were inoculated with Penicillium roqueforti spores.

The addition of $P$. roqueforti spores simulated heavily contaminated cheese. The 
purpose of inoculating cheese with mold spores was to see how natamycin and derivative concentrations are affected during spoilage. Penicillium roqueforti was selected as the inoculation organism because of its prevalence as a spoilage organism in the dairy industry. Bullerman (1981) reported that $82 \%$ of molds on refrigerated cheese belongs to the genus Penicillium. The specific Penicillium strain selected, Penicillium roquefortii Thom, was isolated from a Cheddar cheese substrate in Australia (ATCC, Manassas, VA).

The objective of this study was to gain insight on the stability and degradation of natamycin and a natamycin semisynthetic derivative during typical storage conditions for shredded cheese. The effects of antibiotic application, MAP, open package conditions, and mold growth on natamycin and the derivative's stability were examined.

\section{Materials and Methods}

\section{Spore Stock Preparation}

Penicillium roquefortii Thom (ATCC \#52322) (ATCC, Manassas, VA) was cultured in Malt Extract Broth (MEB) (Difco, Detroit, MI) and then plated on Malt Extract Agar (MEA) (Difco, Detroit, MI). Conidia and ascospores were harvested by washing each plate with $10 \mathrm{ml}$ of sterile potassium phosphate : magnesium chloride diluent (PMC) $+1 \%$ Tween 80 diluent and gently rubbing surface with a sterile glass spreader (Nielsen et al. 1988). Suspensions were pipetted into sterile centrifuge tubes. Washing procedure was repeated two more times for a total of three washes per plate. Samples were vortexed for 2 minutes and decanted through sterile glass wool into a sterile $250 \mathrm{ml}$ flask. After thorough mixing, $25 \mathrm{ml}$ aliquots of spore suspension were transferred into sterile centrifuge tubes. Tubes were centrifuged for 10 minutes on low speed without braking (Sorvall® Inc., RC-5B centrifuge (Newtown, CT). Supernatant was removed and $10 \mathrm{ml}$ of MEB was added to the centrifuge tubes. Mixture was then transferred to a $250 \mathrm{ml}$ sterile flask. After thorough mixing, $1 \mathrm{ml}$ spore suspensions were placed in $2 \mathrm{ml}$ cryogenic tubes and immersed in liquid nitrogen for 24 hours. Cryogenic tubes were stored in cryogenic freezer at $-80^{\circ} \mathrm{C}$ prior to lyophilization (Forma Scientific, Inc., Model 5479 
freezer, Marjetta, $\mathrm{OH})$.

Spores were freeze dried in laboratory freeze dryer at $25^{\circ} \mathrm{C}$ for 24 hours to remove all water (Virtis, Gardiner, New York). The concentration of spores per gram was determined by enumerating on Dichloran Rose Bengal (DRB) Agar (Oxoid Unipath, Ogensburg, NY) supplemented with $0.1 \%$ chloramphenicol (Aldrich, Milwaukee, WI) (Ellis et al. 1993). A spore stock cellulose powder was made by adding the appropriate amount of freeze dried spores to common-type cellulose.

\section{Sample Production}

One month aged Cheddar cheese (Alto Dairy, Black Creek, WI) was shredded by a electric shredder (Paxton Corp., Shelton, CT) in $2 \mathrm{~kg}$ batches. A 2\% w/w cellulose mixture (40 g) (Gist-brocades, Menomonee Falls, WI) was added to each of the 11 application treatments and tumbled for 2 minutes in a $110 \mathrm{~L}$ fabricated automated tumbler. The cellulose mixture in five of the application treatments was spiked with approximately $2 \times 10^{5} \mathrm{P}$. roqueforti spores. The addition of these spores targeted a final concentration of $10^{2} \mathrm{CFU} / \mathrm{g}$ for the shredded cheese.

During tumbling, a total of $1 \%(\mathrm{w} / \mathrm{w})$ water $(20.4 \mathrm{~g})$ was sprayed into the tumbler and onto the cheese. Incorporated into the $1 \%$ water spray was the appropriate concentrations of natamycin (Aldrich, Milwaukee, WI) and derivative. No antimycotic was added to the $1 \%$ water for $0 \mathrm{ppm}$ treatments, but for 10 and $20 \mathrm{ppm}$ treatments, the $1 \%$ water addition included 0.1 and $0.2 \%$ antimycotic agent.

From the $2 \mathrm{~kg}$ batch, $66 \mathrm{~g}$ samples were placed in packaging bags (Cryovac Corp., Duncan, SC). Bags were MAP using a proportional gas blender (Smith Equipment, Model 299037F, Watertown, SD) and vacuum packaging machine (Koch, Model X-200, Kansas City, MO). Gas mixture was comprised of 75\% nitrogen and 25\% carbon dioxide. Each package was examined for leakage by a fabricated chambered gas leak detector. Samples were stored at $4{ }^{\circ} \mathrm{C}$ in a portable walk-in refrigerator (W.H. Porter Inc. Model 1200, Holland, MI). Three replications of this experiment were conducted over a three day period. 


\section{Procedure for Determining Natamycin and Derivative Concentration on Cheese}

Packages were opened after 0, 15, and 30 days MAP storage. Opened packages were sampled after 0 and 20 days. Natamycin and derivative concentration of shredded cheese samples was determined using a spectrometric method presented by De Ruig (1987) and outlined in IDF 140A:1992 with several modifications (IDF, 1992). The sample size was increased to $15 \mathrm{~g}$ from $5 \mathrm{~g}$. Proportional increases in extraction liquids, methanol and water, were adjusted accordingly. A

$15 \mathrm{~g}$ control sample incorporated with $2 \%$ cellulose by weight, but no preservative agents was used to establish the spectrometric baseline

\section{Results and Discussion}

Antibiotic treatments targeted 10 and $20 \mathrm{ppm}$ of natamycin and the derivative on the cheese surface. Initial concentrations, measured at 0 days MAP : 0 days open, deviated from target concentrations by an average of 6 and $11 \%$ for natamycin and derivative. Antibiotic concentration for non-inoculated treatments decreased linearly during storage independent of MAP or open conditions (Figure 1). No trend could be established for inoculated treatments (Figure 2). The $10 \mathrm{ppm}$ natamycin non-inoculated treatment showed a loss of $19 \%$ after 30 days of MAP storage (Figure 3). The loss was $15 \%$ for the $10 \mathrm{ppm}$ natamycin inoculated treatment. (Figure 3). Examining the same conditions for the derivative showed that its degradation was much greater. The $10 \mathrm{ppm}$ derivative treatments for non-inoculated and inoculated samples showed losses of 25 and $47 \%$ after 30 days of MAP storage (Figure 3). Analysis of the $20 \mathrm{ppm}$ concentrations showed that degradation of the derivative was less than that of natamycin. The $20 \mathrm{ppm}$ natamycin treatments for non-inoculated and inoculated samples had identical losses of $33 \%$ after 30 days of MAP storage (Figure 3). For the same storage conditions, the $20 \mathrm{ppm}$ derivative treatment for non-inoculated and inoculated samples showed losses of 25 and $17 \%$.

Results indicate that the derivative showed a greater percent loss at a concentration of 10 
ppm, but a lower percent loss at a concentration of $20 \mathrm{ppm}$. Degradation for the $20 \mathrm{ppm}$ derivative treatment may have been slowed as a result of its solubility. Although natamycin is most stable in a crystalline powder form, micellular suspensions of natamycin offer protection by shielding the labile sites of the molecule (Thomas, 1976). Assuming that the derivative would behave similarly to natamycin, the increased solubility of the derivative would provide additional protection. This increased protection may have not been noticeable until antibiotic concentrations reached $20 \mathrm{ppm}$.

The degradation of natamycin is nearly complete after it has been opened for 20 days. Percent loss values for natamycin and the derivative were mostly negative and varied when comparing antibiotic loss for open conditions between MAP samples. No degradation pattern was established when comparing antibiotic concentration for storage conditions: 0 days MAP : 20 days open, 15 days MAP : 20 days open, and 30 days MAP : 20 days open (Figure 3).

A much more informative comparison of antibiotic concentration was seen when the percent loss of natamycin and the derivative were measured after 20 days open for each MAP storage condition. A negative linear trend was observed when examining these results. Noninoculated treatments opened for 20 days and stored at 0 days MAP showed losses of 24, 33, 24, and $27 \%$ for $10 \mathrm{ppm}$ natamycin, $10 \mathrm{ppm}$ derivative, $20 \mathrm{ppm}$ natamycin, and $20 \mathrm{ppm}$ derivative treatments (Figure 4). Antibiotic losses were greater for inoculated treatments stored at the same conditions. The $10 \mathrm{ppm}$ natamycin, $10 \mathrm{ppm}$ derivative, $20 \mathrm{ppm}$ natamycin, and $20 \mathrm{ppm}$ derivative inoculated treatments showed antibiotic losses of 48, 46, 25, and 36\% (Figure 4). The rate of antibiotic degradation for all antibiotic concentrations was almost identical for noninoculated treatments (Figure 5). The same was true for inoculated samples (Figures 6)

MAP samples stored for 15 days and opened for 20 days had lower percent loss values then MAP samples stored for 0 days and opened for 20 days. This confirms the observation that antibiotic degradation occurs during MAP storage. The $10 \mathrm{ppm}$ natamycin, $10 \mathrm{ppm}$ derivative, 20 ppm natamycin, and 20 ppm derivative non-inoculated treatments had antibiotic losses of 18, 15, 22, and 27\% (Figure 4). Inoculated treatments for these same antibiotic concentrations had antibiotic losses of 21, 27, 13, and 5\% (Figure 4). The rate of antibiotic degradation for all antibiotic concentrations was again similar for non-inoculated and inoculated samples (Figures 7 
and 10).

Percent loss values further decreased for MAP samples stored for 30 days. The $10 \mathrm{ppm}$ natamycin, 10 ppm derivative, 20 ppm natamycin, and 20 ppm derivative non-inoculated treatments had antibiotic losses of 0,8 , and 1\% (Figure 4). Inoculated treatments for these same antibiotic concentrations had antibiotic losses of 27, $-5,-13$, and 2\% (Figure 4). The rate of antibiotic degradation had a linear trend for the non-inoculated samples, but inoculated samples showed no degradation trend.

The rate of antibiotic degradation was affected by the degree of spoilage. Samples heavily contaminated with mold showed a faster rate and greater loss of natamycin and derivative during MAP and open storage conditions. This result was anticipated. The outgrowth of mold in packages may cause stress on the tetraene moiety of the antibiotics. In addition, mold growth may amplify oxidative degradation. Some metabolic byproducts of fungal organisms are chemical oxidants which severe the interconnected, unsaturated double bonds of the antibiotics (Raab, 1972).

The stability of natamycin and the derivative was compromised somewhat greater during opened conditions then MAP conditions. Extensive degradation of both antibiotics did however occur during MAP storage. Antibiotic concentrations were nearly identical for non-inoculated samples stored at 0 days MAP : 20 days open and 30 days MAP : 0 days open. The rate of degradation slowed tremendously for MAP samples stored longer than 15 days. These results indicate that regardless of initial concentration, antibiotic agents reach a equilibrium within the MAP package after 15 days. Little antibiotic degradation occurs after this equilibrium is established. The stability of natamycin and the derivative were similar. Review of Figures 1, 2, $5,6,7$, and 8 confirm this statement.

\section{Conclusions}

The rate and loss of natamycin and derivative is increased for heavily contaminated samples. The outgrowth of mold may cause stress on the tetraene moiety of the antibiotics and cause the severing of interconnected, unsaturated double bonds. The severing of these bonds 
renders natamycin and the derivative inactive. Similar degradation can occur in the presence of chemical oxidants. Oxidative degradation may have occurred in the presence of chemical oxidants produced by the metabolic byproducts of fungal organisms.

Natamycin and derivative degradation occurred nearly identical in MAP and open conditions. This finding suggests that increased oxygen concentrations do not amplify antibiotic degradation. It also suggests that a MA of $75 \%$ nitrogen and $25 \%$ carbon dioxide is just as destructive to natamycin and natamycin synthetics as opened conditions.

Degradation caused by ultraviolet light was not discussed in this paper. The inactivation of natamycin by ultraviolet light does not show decreases in maxima peaks $279,290,304$, and $318 \mathrm{~nm}$ and therefore would not be detected by analytical tests utilized in this study. It is hypothesized that the photodynamic destruction of the derivative is similar to one discussed for natamycin. 
Figures

Figure 1: Antibiotic concentration of 1 month aged shredded Cheddar cheese for noninoculated treatments vs. total days of storage

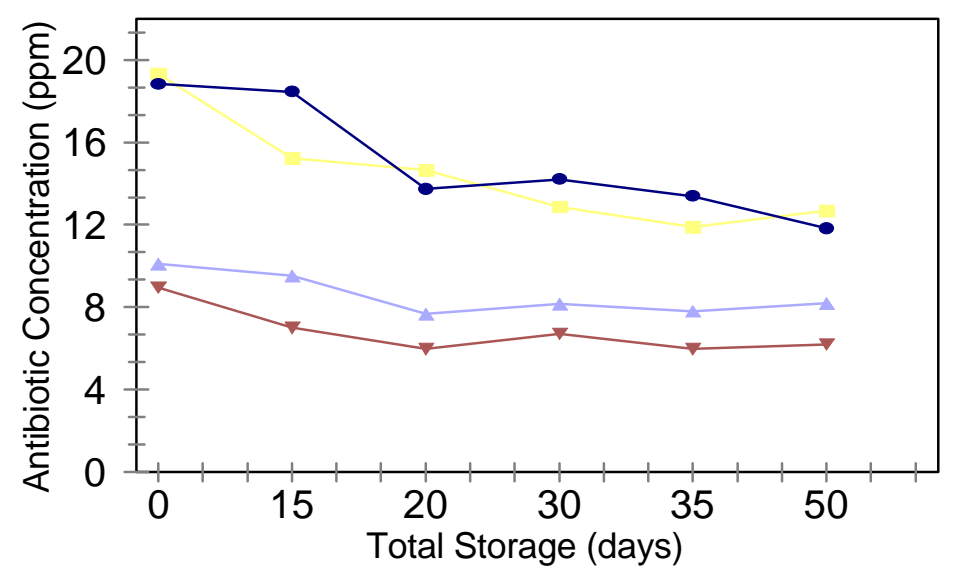

$$
\begin{array}{r}
-10 \text { ppm Natamycin } \rightarrow 10 \text { ppm Derivative } \\
-20 \text { ppm Natamycin } \multimap 20 \text { ppm Derivative }
\end{array}
$$

Figure 2: Antibiotic concentration of 1 month aged shredded Cheddar cheese for inoculated treatments vs. total days of storage

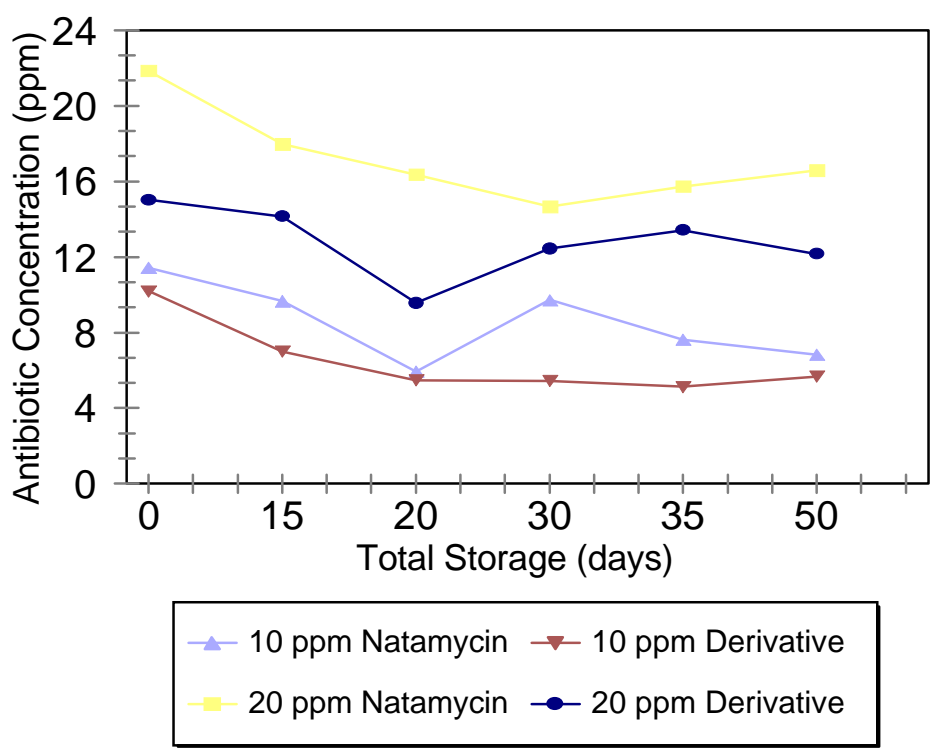


Figure 5: Antibiotic concentration of 1 month aged shredded Cheddar cheese for noninoculated treatments at storage condition: 0 Days MAP : 0, 20 Days Open

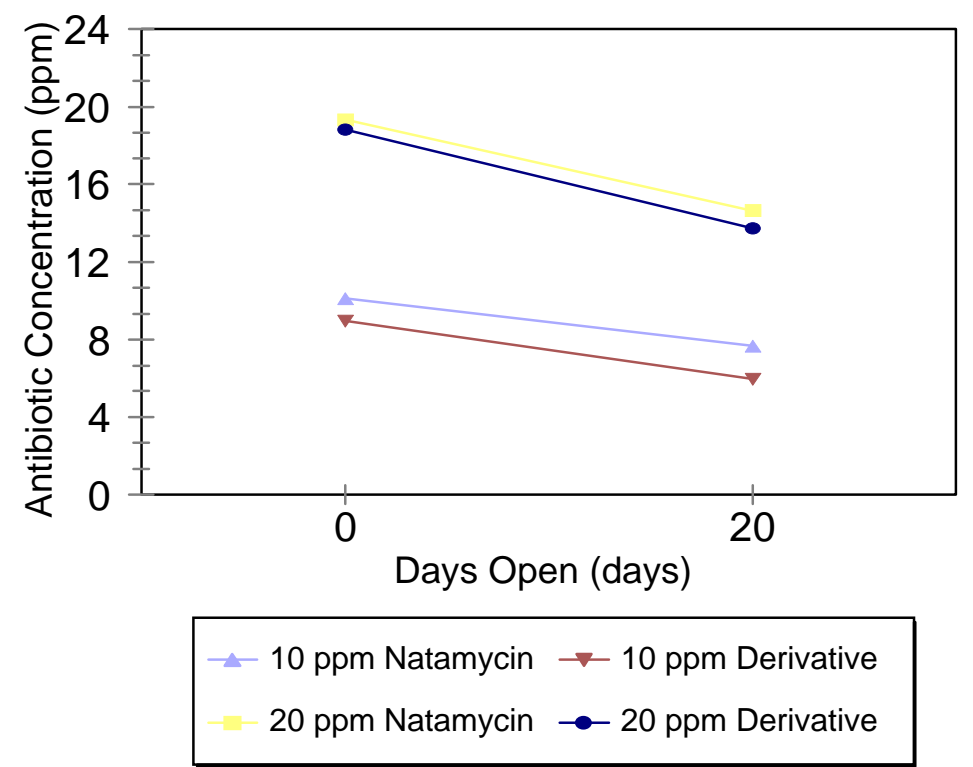

Figure 6: Antibiotic concentration of 1 month aged shredded Cheddar cheese for inoculated treatments at storage conditions: 0 Days MAP : 0, 20 Days Open

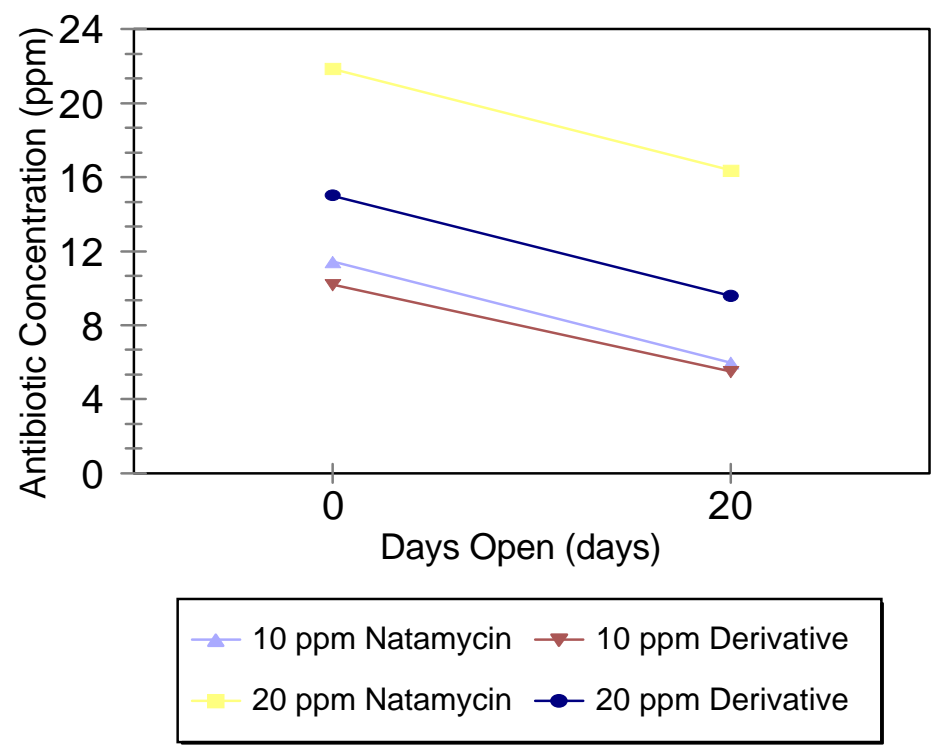


Figure 7: Antibiotic concentration of 1 month aged shredded Cheddar cheese for noninoculated treatments at storage condition: 15 Days MAP : 0, 20 Days Open

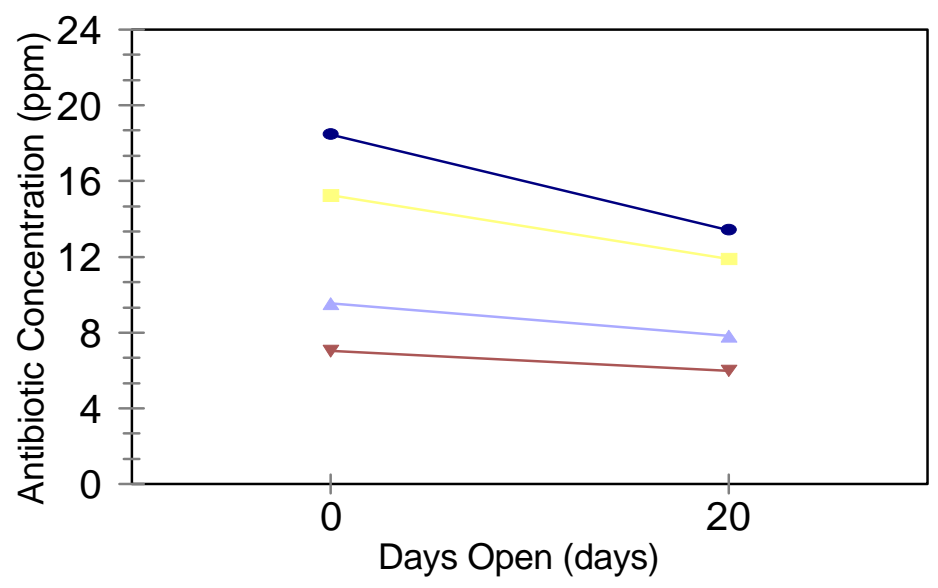

$\triangle 10$ ppm Natamycin $\rightarrow 10$ ppm Derivative 20 ppm Natamycin $\bullet 20$ ppm Derivative

Figure 8: Antibiotic concentration of 1 month aged shredded Cheddar cheese for inoculated treatments at storage condition: 15 Days MAP : 0, 20 Days Open

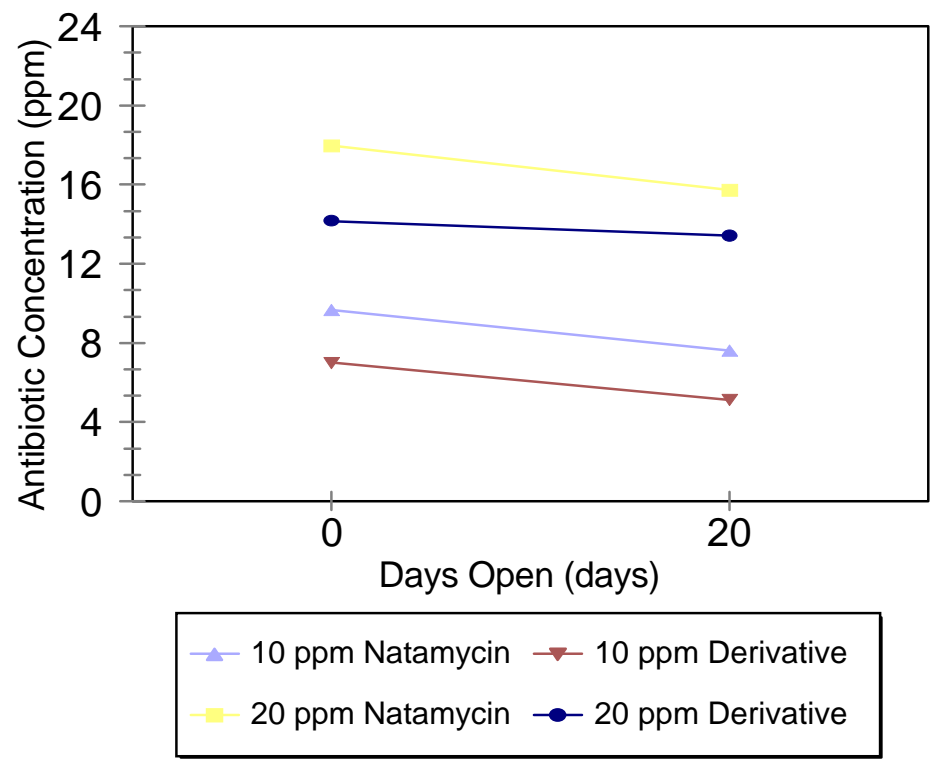


Figure 9: Antibiotic concentration of 1 month aged shredded Cheddar cheese for noninoculated treatments at storage condition: 30 Days MAP : 0, 20 Days Open

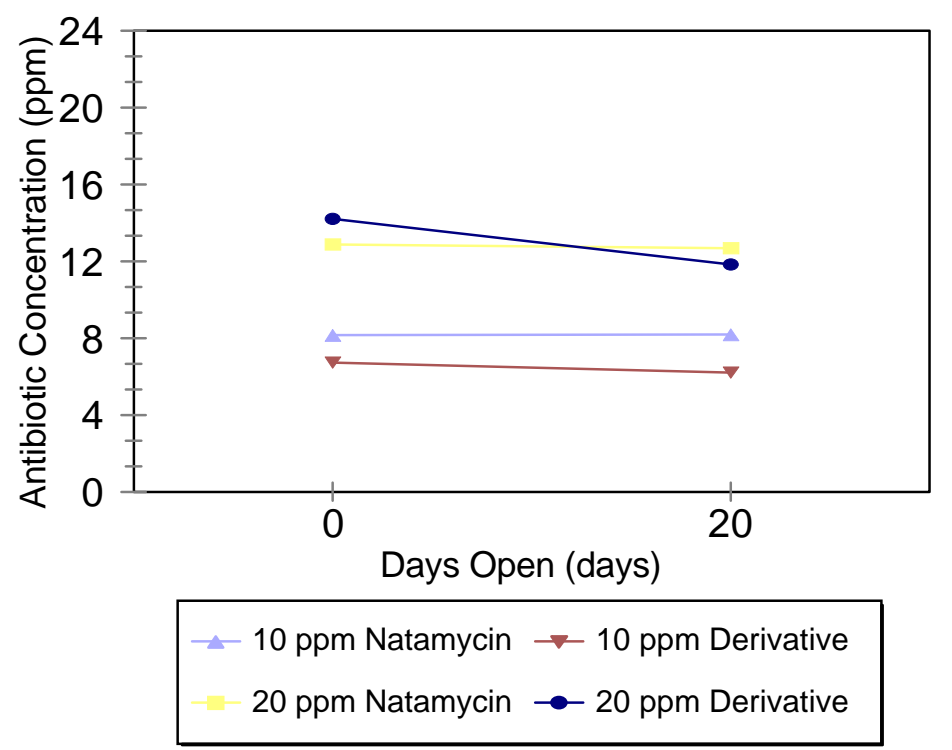

Figure 10: Antibiotic concentration of 1 month aged shredded Cheddar cheese for inoculated treatments at storage condition: 30 Days MAP : 0, 20 Days Open

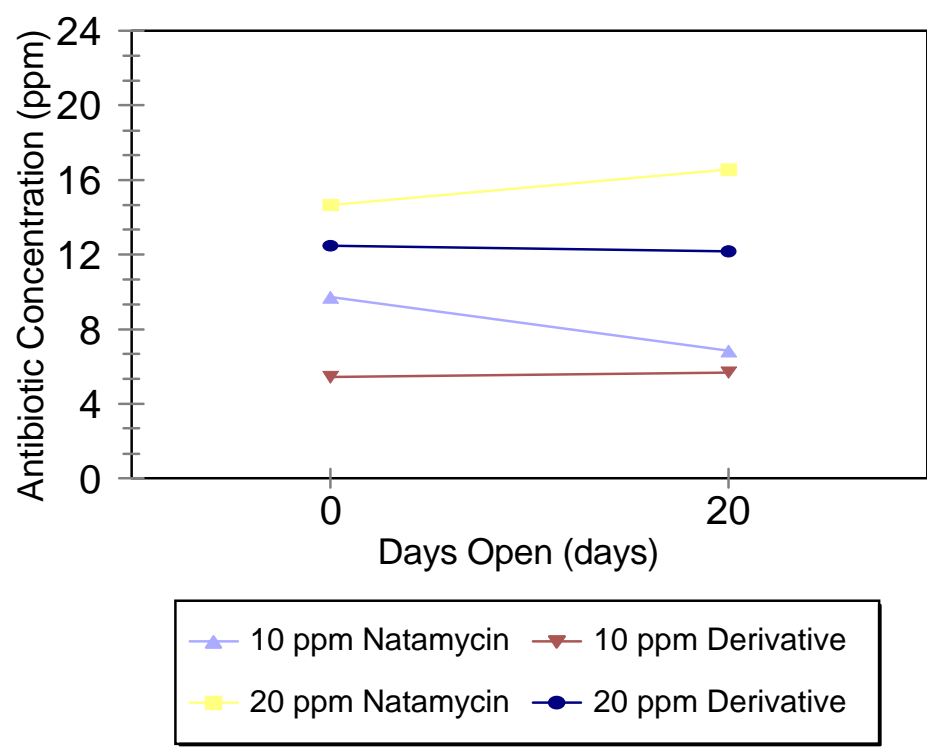




\section{References}

Barr, F.S. 1959. Tennecetin, a new antifungal antibiotic. Toxicological Studies. Antib. And Chemother. 9:406-410.

Bullerman, L.B. 1981. Public health significance of molds and mycotoxins in fermented dairy products. J. Dairy Sci. 64:2439-2452.

Dekker, J. and Ark, P.A. 1959. Protection of the antibiotic pimaricin from oxidation and ultraviolet light by chlorophyllin and other compounds. Antib. And Chemother. 9:327-332

Ellis, W.O., Smith, J.P., Simpson, B.K., and Ramaswamy, H. 1993. Effect of inoculum level on aflatoxin production by Aspergillus flavus under modified atmosphere packaging (MAP) conditions. Food Microbiol. 10:9-12.

IDF. 1992. International Dairy Federation Standard 140A:1992 - Cheese and cheese rind determination of natamycin content - method by molecular absorption spectrometry and by high performance liquid chromatography.

Oostendorp, J.G. 1981. Natamycin. Antonie van Leeuwenhoek. 47:170-171.

Nielsen, R.V., Beuchat, L.R., and Frisvad, J.C. 1988. Growth of and fumitremorgin production by Neosartorya fischeri as affected by temperature, light, and water activity. Appl. Environ. Microbiol. 54:1504-1510.

Posthuma, J. and Berends, W. 1960. Triplet-triplet transfer as a mechanism of a photodynamic reaction. Biochim. Biophys. Acta. 41:538-541. 
Posthuma, J. and Berends, W. 1961. Energy transfer in aqueous solutions. Biochim. Biophys. Acta. 51:390-392.

Ruig, W.G. de. 1987. Determination of natamycin in cheese and cheese rind: Interlaboratory collaborative study. J. Assoc. Off. Anal. Chem. 70:949-955.

Thomas, A.H. 1976. Analysis and assay of polyene antifungal antibiotics. The Analyst. 101:321339. 


\section{VITA}

The author, Eric C. Suloff, was born on January 17, 1975 in Reading, Pennsylvania. He is the son of Charles and Christine Suloff who reside in Allentown, Pennsylvania. Eric attended Parkland High School in Orefield, Pennsylvania and received his diploma in June of 1993. In May 1997, he received his B.S. degree in Food Science from the Pennsylvania State University, in State College, Pennsylvania.

In August of 1997, he entered Virginia Polytechnic Institute and State University to begin graduate study in pursuit of a Master of Science degree in Food Science and Technology. Eric married his high school sweetheart and longtime friend, Amy Lynn, in July 1998. Eric and Amy reside in Salem, Virginia and are anxiously awaiting the arrival of their first child at the time of this writing. 